\title{
DERIVING INFORMATION FROM SPATIAL SAMPLING FLOOR- BASED PERSONNEL DETECTION SYSTEM
}

A Thesis
Presented to
The Faculty of the Graduate School
At the University of Missouri-Columbia
In Partial Fulfillment
Of the Requirements for the Degree
Doctor of Philosophy
Dr. Harry W. Tyrer, Thesis Supervisor
May 2017
By MUHEIDAT


The undersigned, appointed by the dean of the Graduate School, have examined the

thesis entitled

\title{
DERIVING INFORMATION FROM SPATIAL SAMPLING FLOOR- BASED PERSONNEL DETECTION SYSTEM
}

\author{
Presented by Fadi Muheidat,
}

A candidate for the degree of Doctor of Philosophy

And hereby certify that, in their opinion, it is worthy of acceptance.

Dr. Harry Tyrer

Dr. Justin Legarsky

Dr. Zhihai He

Dr. Mihail Popescu 


\section{DEDICATION}

This dissertation is dedicated to my daughter Kinda, my son Yaman, my wife Tahani, and to all my family and friends. 


\section{ACKNOWLEDGEMENTS}

I would like to express my appreciation to everyone who helped me complete this work in any aspect. Firstly, I would like to express my sincere gratitude and special thanks to Professor Harry Tyrer for the continuous support, guidance, motivation, and for his valuable guidance and patience. His guidance helped me in every aspect of my schoolwork, research and teaching. His immense knowledge and expertise enriched my work and supported me in writing my thesis.

Besides my advisor, I would like to thank the rest of my thesis committee: Dr. Justin Legarsky, Dr. Mihail Popescu, and Dr. Zhihai He, for their acceptance to serve in my thesis committee and for the insightful comments and valuable guidance during my comprehensive exam. I would like to extend my thanks to Dr. Mihail Popescu for helping for the insightful comments, and suggestions to explore other perspectives in my research, and to improve this work.

I would like to thank Dr. Marjorie Skubic for her advice and assistance. My deep gratitude to Andrew Craver (Andy) from Tiger place for his support and helping me use the GAITRite mat and software.

Last but not the least; I would like to give my heartfelt appreciation to my wife, Tahani, my daughter Kinda and my son Yaman for their support and patience along this journey. My deepest thanks to my parents, Ali and Laila, and my in-laws, Suleiman and Samira and my brothers and sisters for their continual support, encouragement and love. 


\section{TABLE OF CONTENTS}

ACKNOWLEDGEMENTS ................................................... ii

LIST OF FIGURES.......................................................... viii

LIST OF TABLES ..............................................................

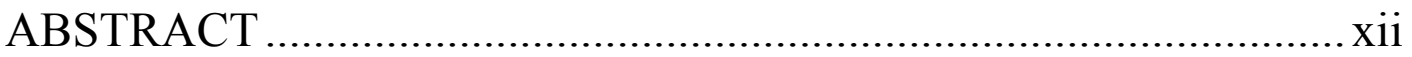

1 Chapter 1: Introduction ..................................................... 1

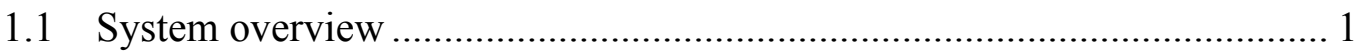

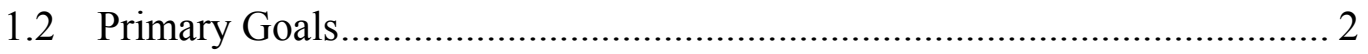

2 Chapter 2: Background ................................................ 5

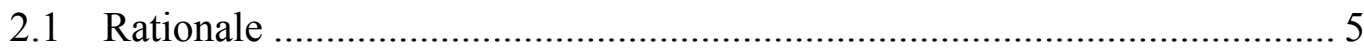

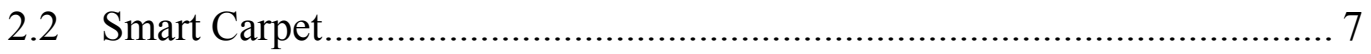

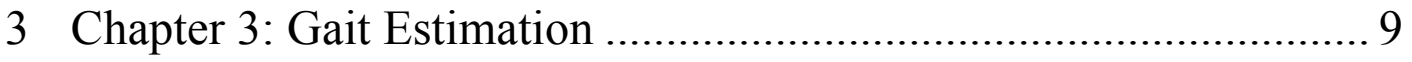

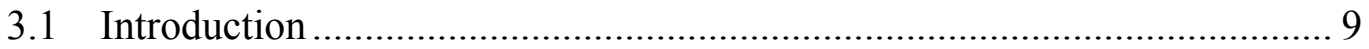

3.2 Manuscript: Estimating Walking Speed, Stride Length, and Stride Time using a Passive Floor Based Electronic Scavenging System.................................... 10

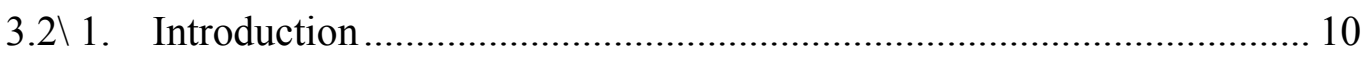

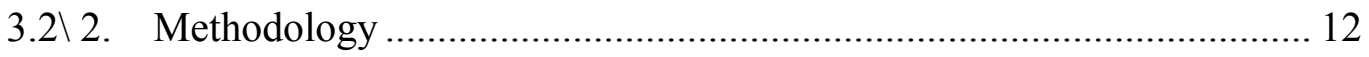


3.2 3. Experimental Results ..................................................................... 18

3.2 4. Discussion and conclusion ............................................................... 20

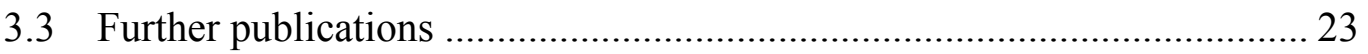

3.4 Details and additional work not yet published …………………................ 24

3.4.1 Preliminary experiments.............................................................................. 24

3.4.2 Subjects Results.................................................................................. 27

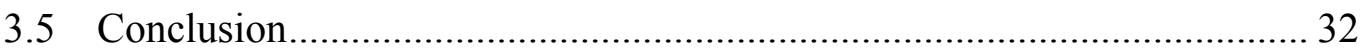

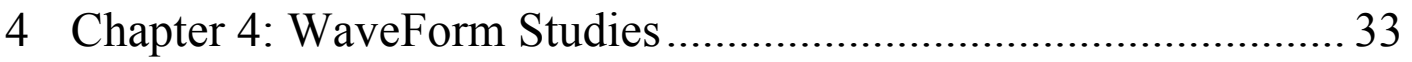

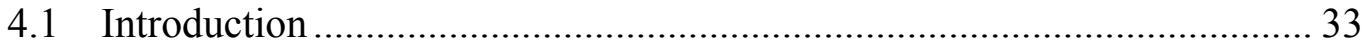

4.2 Waveform Characteristics of Scavenged Charge ....................................... 34

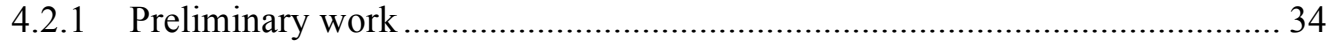

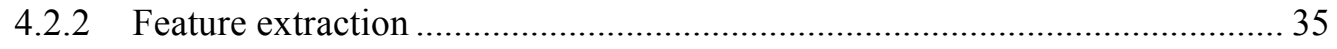

4.2.3 Experimental results and Discussion .......................................................... 40

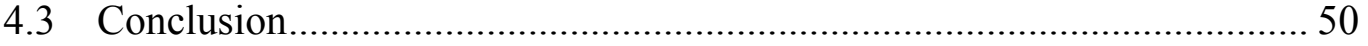

5 Chapter 5: Multiple People Count ….......................................... 51

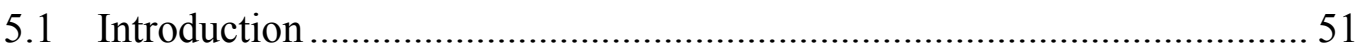

5.2 Manuscript: Counting Multiple People on a Floor Based Array sensor system $\quad 52$

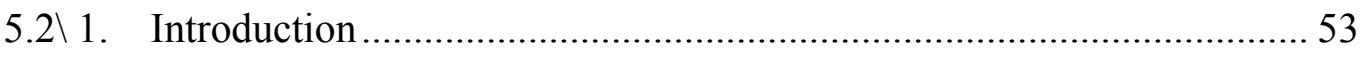

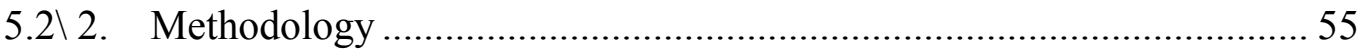

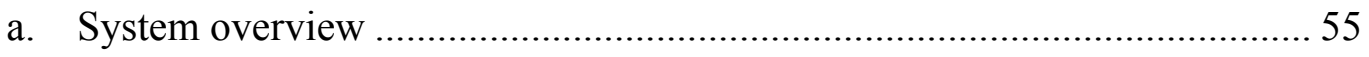

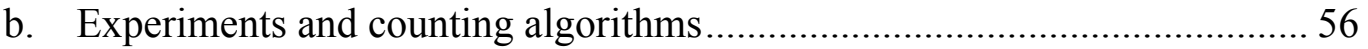


5.2 3. Experimental Results ……………………………......................... 58

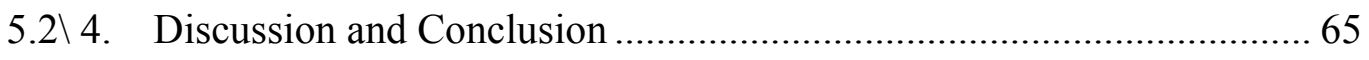

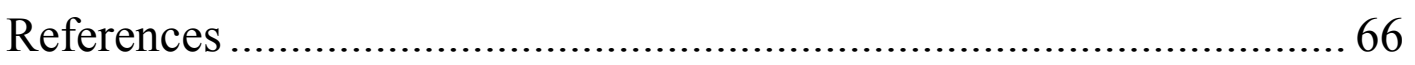

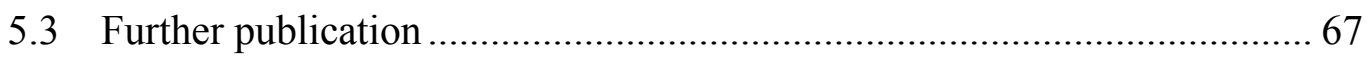

5.4 Details and additional work not yet published ............................................ 67

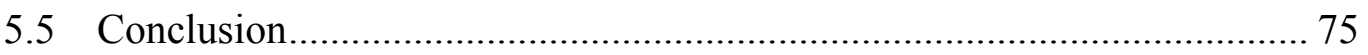

6 Chapter 6: Additional Intelligence, Gait Estimation, and Scavenging

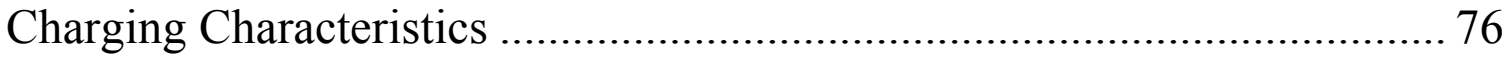

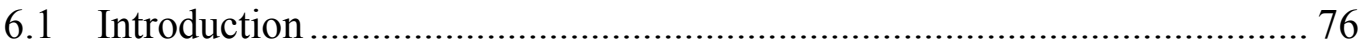

6.2 Manuscript: Floor Based Sensor System: Additional Intelligence, Gait Estimation, and Scavenging Charging Characteristics ........................................... 77

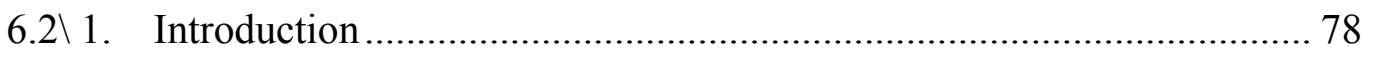

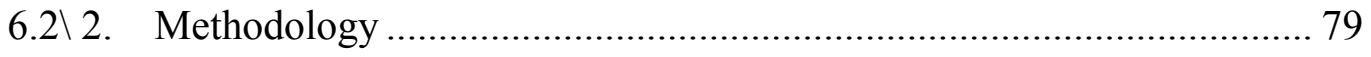

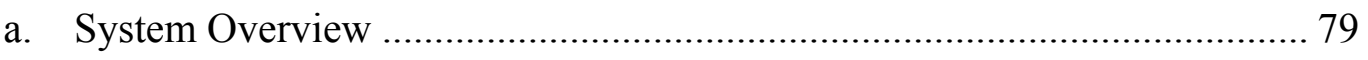

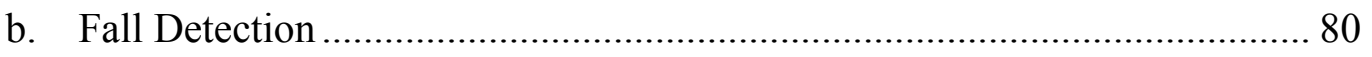

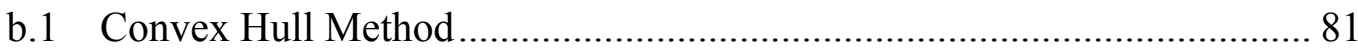

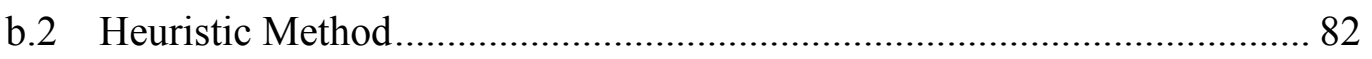

c. Gait Estimation Performance ..................................................................... 82

d. Signal Scavenging Charging Characteristics ………………......................... 85

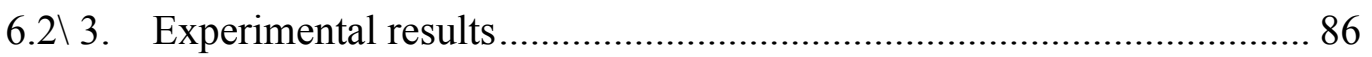

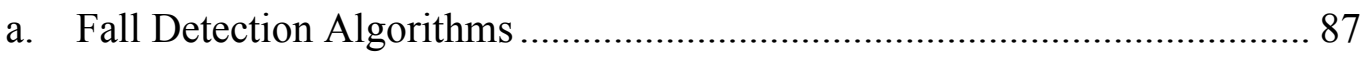


b. Gait Estimation System Performance ............................................................... 87

b. Scavenged Charge Characteristics …………………................................. 90

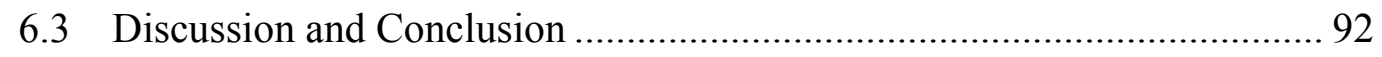

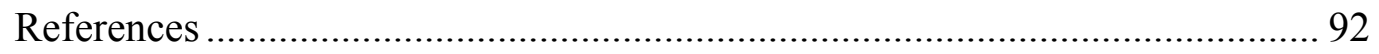

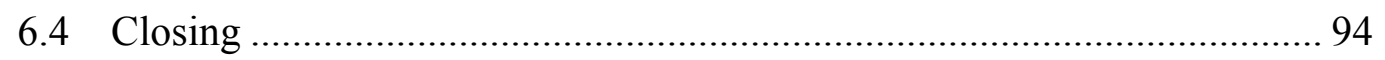

7 Deriving Information from Low Spatial Resolution Floor-Based

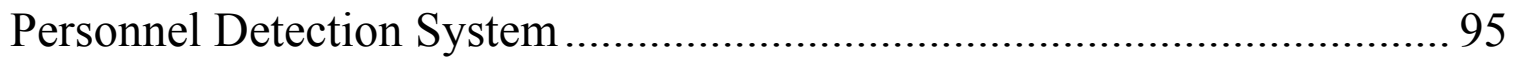

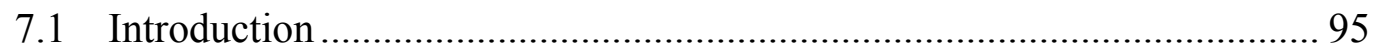

7.2 Manuscript: Deriving Information from Low Spatial Resolution FloorBased Personnel Detection System....................................................................... 96

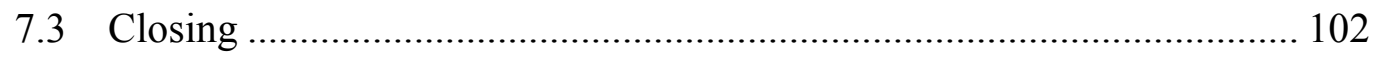

8 Chapter 6: Discussion and Conclusion .......................................... 103

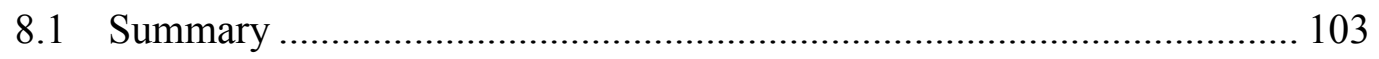

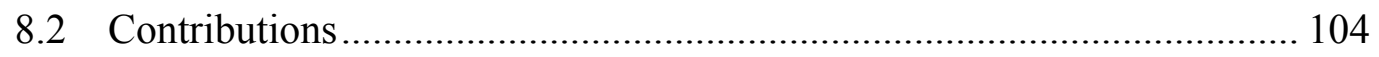

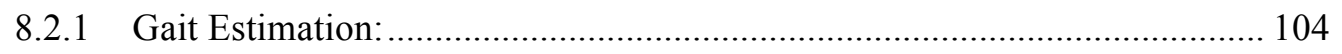

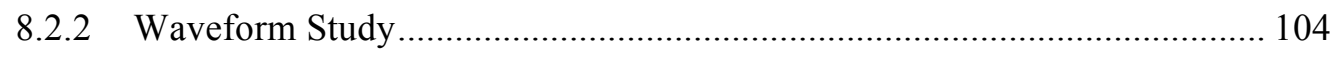

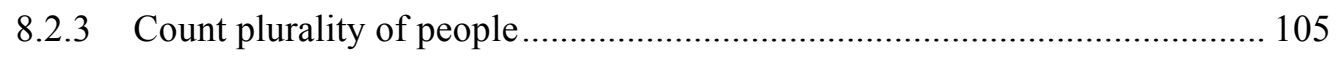

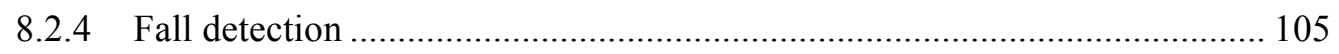

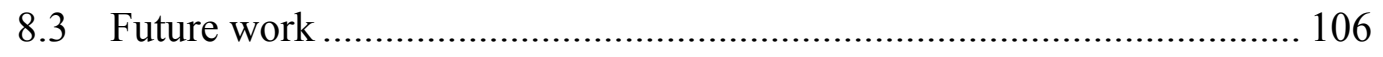

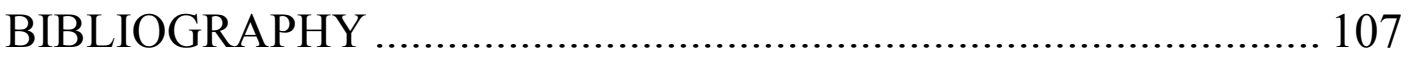




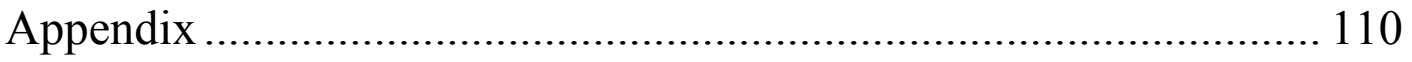

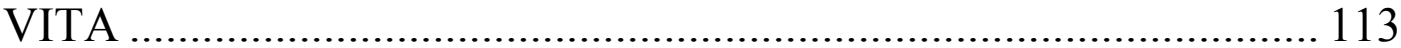




\section{LIST OF FIGURES}

Figure $\quad$ Page

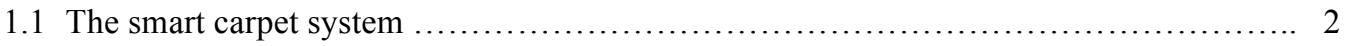

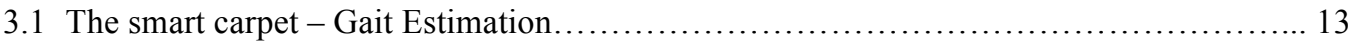

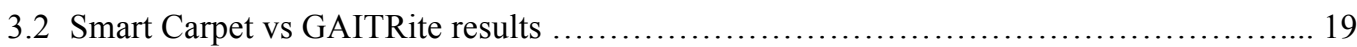

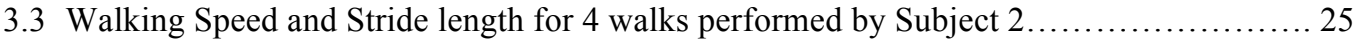

3.4 Box plot of Gait Parameters for Smart Carpet and GAITRite (4 subjects)................. 26

3.5 Box plot of Gait Parameters for Smart Carpet and GAITRite ( 9 subjects)...................31

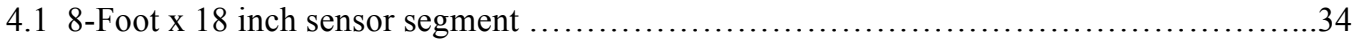

$4.218 \times 18$ inches sensor segment; layout, foot, generated signal …..........................35

4.3 Sample waveforms generated with subject wearing shoes, and barefooted ............... 37

4.4 Step by step processing of the scavenged signal classification ......................... 37

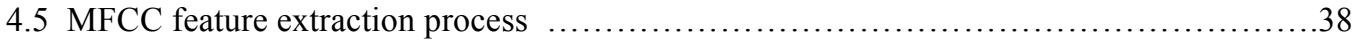

4.6 Samples of scavenged signal (vinyl, cloth, tiles ) surfaces...........................42

4.7 Total power computed for the walks wearing shoes, socks, and barefooted ...............43

4.8 The average walking speed for subject walking wearing shoes, and barefooted ............49

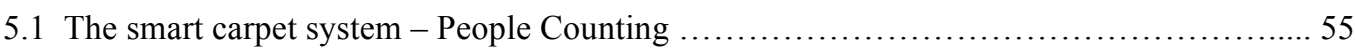

5.2 Carpet layout: Active segments A, B and C. Sensors in $\mathbf{D}$ are turned off..................56

5.3 Active sensors map: Two people walk in Opposite directions ...........................59

5.4 Active sensors map: Two people walk in same directions ............................59

5.5 Active sensors map: Four people walk in Opposite directions ...........................59

5.6 Active sensors map: Full walk $(3,4)$ people walk in same and opposite directions ..........60

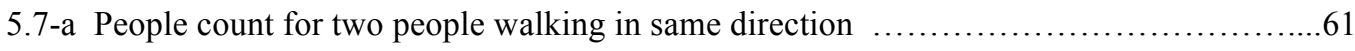

5.7-b People count for three people walking in same direction .............................61

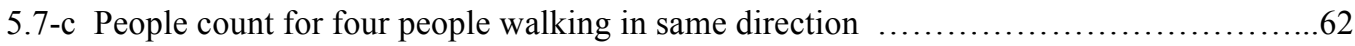

5.8 People count for 10 walks for (WS=9) frames for the Two, Three, Four people ............62

5.9 Individual walk trials in transverse direction. Segment D sensors turned OFF _............63 viii 
5.10 the count of subgroups formed at differing sliding window sizes (WS) for the scenario of two (top), three (middle), and four (bottom) people walking in the same direction

5.11 the count of subgroups formed at differing sliding window sizes $(W S)$ for the scenario of two (top), and four (bottom) people walking in the opposite direction.

5.12 the count of people for two, three, and four people walking in the same and opposite

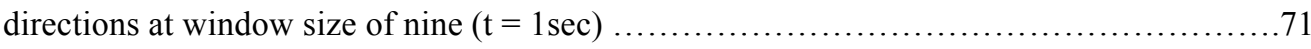

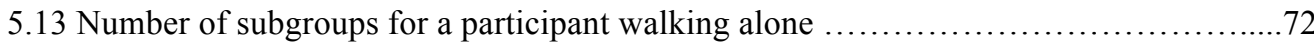

5.14 Number of subgroups for two participants walking same direction ...................73

5.15 Number of subgroups for two participants walking opposite direction …..............73

5.16 Number of subgroups for four participants walking same direction .................. 74

5.17 Number of subgroups for four participants walking opposite direction .................74

6.1 Smart Carpet full system- gait Estimation and Fall detection ......................... 80

6.2 Walk-Fall pattern: Each rectangle represents one frame $\ldots \ldots \ldots \ldots \ldots \ldots \ldots \ldots \ldots \ldots \ldots \ldots 1$

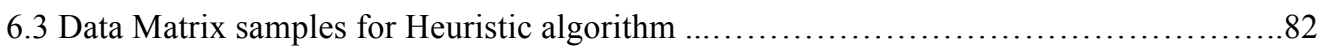

6.4 Histogram of Gait parameters fitted with normal distribution function .................83

6.5 Scatter plot with fitting line of Gait parameters for smart carpet and GAITRite ..........89

6.6 Scavenged signals for adult male walking on sensors covered by carpet (a) Vinyl (b) ...91

7.1 Smart Carpet full system- gait Estimation and Fall detection, people counting...........97 


\section{LIST OF TABLES}

Table

Page

3.1 Difference in walking speed, stride time and length ......................19

3.2 Difference in Walking Speed Smart Carpet and GAITRite)...............20

3.3 Difference in Stride Time Smart Carpet and GAITRite)....................21

3.4 Difference in Stride Length Smart Carpet and GAITRite)................21

3.5 The average of the gait parameters for 4 subjects $\ldots \ldots \ldots \ldots \ldots \ldots \ldots \ldots . \ldots . \ldots . \ldots . \ldots$

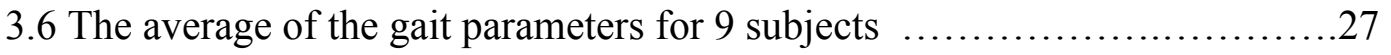

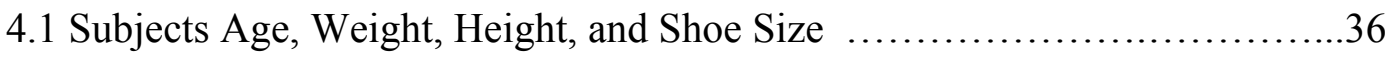

4.2 Average power measured using Vinyl, cloth, and tiles....................40

4.3 Confusion matrix subjects wearing Shoes $(60 \%$ training $) \ldots \ldots \ldots \ldots \ldots \ldots . . . \ldots 45$

4.4 Confusion matrixsubjects Barefooted (60\% training).....................45

4.5 Confusion matrix subjects wearing Shoes $(10 \mathrm{FXV}) \ldots \ldots \ldots \ldots \ldots \ldots \ldots \ldots \ldots \ldots$

4.6 Confusion matrix subjects Brefooted (10FXV) ......................46

4.7 Confusion matrix trained wearing shoes, tested with Barefooted ...........47

4.8 Confusion matrix both wearing shoes and Barefooted (10FXV).............48

4.9 Average Walking Speed for both wearing shoes and Barefooted ............49

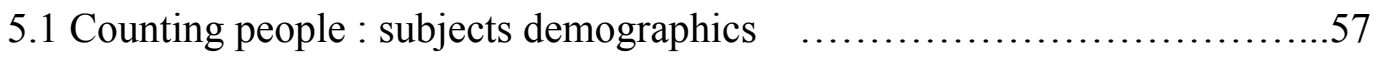

5.2 Counting people : Average active sesnors count $\ldots \ldots \ldots \ldots \ldots \ldots \ldots \ldots . \ldots 64$

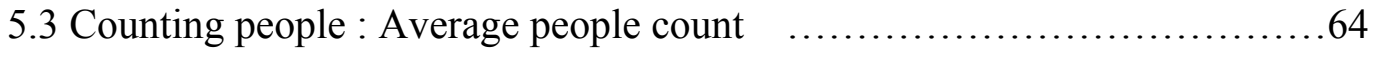

6.1 Statstics for Gait parameters for 75 walks $\ldots \ldots \ldots \ldots \ldots \ldots \ldots \ldots \ldots \ldots . . . \ldots 4$

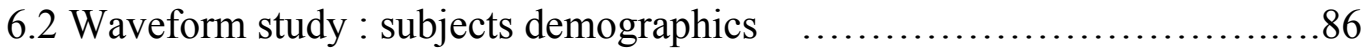


6.3 Fall detection : Performance measures

6.4 Gait Estimation Statistics :ttest-2 .88

6.5 Average power measured using Vinyl, cloth

7.1 Counting people : Average active sesnors and people plurality count.....100

7.2 Gait Estimation : Mean, STDV, and Correlation........................101 


\title{
DERIVING INFORMATION FROM SPATIAL SAMPLING FLOOR-BASED PERSONNEL DETECTION SYSTEM
}

Fadi Muheidat

\author{
Dr. Harry W. Tyrer, Dissertation Advisor
}

\begin{abstract}
Research has shown and identified a clear link between human gait characteristics and different medical conditions. Therefore, a change in certain gait parameters may be predictive of future falls and adverse events in older adults such as physical functional decline and fall risks. We describe a system that is unobtrusive and continuously monitors the gait during daily activities of elderly people. The early assessment of gait decline will benefit the senior by providing an indication of the risk of falls. We developed a low cost floor-based personnel detection system; we call a smart carpet, which consists of a sensor pad placed under a carpet; the electronics reads walking activity. The smart carpet systems is used as a component of an automated health monitoring system, which helps enable independent living for elderly people and provide a practical environment that improves quality of life, reduces healthcare costs and promotes independence. In this dissertation, we extended the functionalities of the smart carpet to improve its ability to detect falls, estimate gait parameters and compared it to GAITRite system. We counted number of people walking on the carpet in order to distinguish the plurality of people from fall event. Additionally we studied the characteristics and the behavior of the sensor's scavenged signal. Results showed that our system detects falls, using computational intelligence techniques, with $96.2 \%$ accuracy and $81 \%$ sensitivity and $97.8 \%$ specificity. The system reliably estimates the gait xii
\end{abstract}


parameters; walking speed, stride length and stride time with percentage errors of $1.43 \%$, $-4.32 \%$, and $-5.73 \%$ respectively. Our system can count the number of people on the carpet with high accuracy, and we ran tests with up to four people. We were able to use computational features of the generated waveform, by extracting the Mel Frequency Cepstral Coefficients (MFCC), and using formal computation intelligence to distinguish different people with an average accuracy of $82 \%$, given that the experiments were performed within the same day.

xiii 


\section{CHAPTER 1: INTRODUCTION}

\subsection{System overview}

The smart carpet system [1,2], shown in Figure 1.1, includes the sensor data acquisition, data manipulating, data reading, storage, display, and communication. The system operates by detecting the person's movement and storing the floor sensor data. The motion on the carpet activates a set of sensors that outputs a voltage signal. The system amplifies the signal, digitizes it, and then translates it into a frame for further processing. The smart carpet data acquisition system scans the sensors at $\mathrm{N}$ frames/second. We parse the frame data into binary image that corresponds to the carpet layout. Where ' 1 ' means the sensor is activated, and ' 0 ' means it is not activated. This image becomes the data structure to perform computations. With the smart carpet, we were able to detect falls using computational intelligence techniques [3]. The software components process the data frames, and use different computational methods process, store, visualize data, and send alerts and notifications. However, because of that good start and promising results, there is a value in investigating and extending the functionality of the smart carpet. This thesis addresses a set of objectives and goals. 


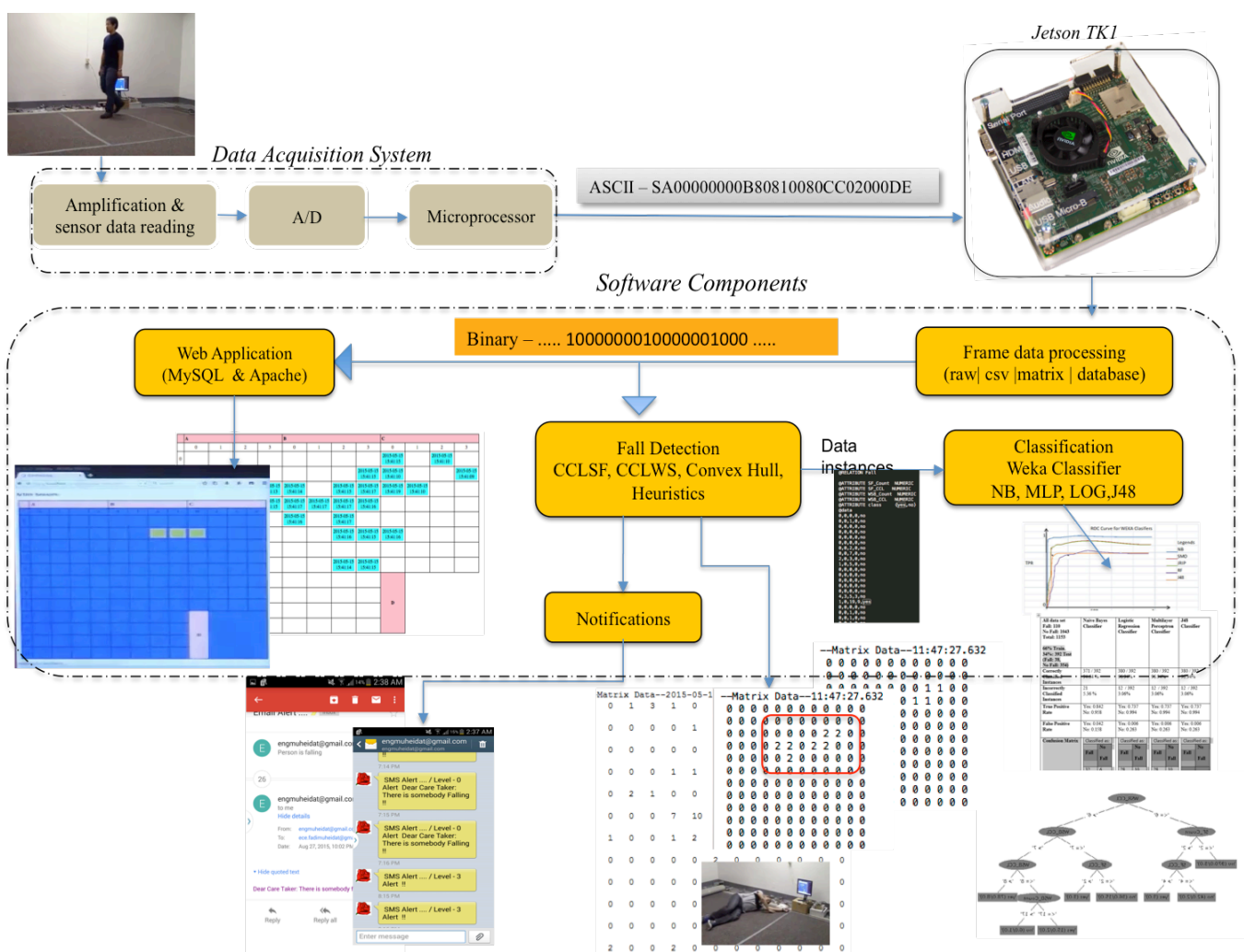

Figure 1-1 The smart carpet system. Data acquisition system, computing power, software components such as Notifications, quasi real-time display, and intelligence classifiers.

\subsection{Primary Goals}

We started with three questions: Can we use the smart carpet to obtain gait estimation? Can we obtain more information from the scavenged sensors signal characteristics? Can we count the plurality of people walking on the carpet? These are the questions we raised and we accomplished them as follows:

We estimated the human gait parameters such walking speed, stride length, and stride time. We counted the number footfalls, and measured the ambulation time, and walking distance. We evaluated the performance of the smart carpet with a ground truth through the use of GAITRite mat. We published a paper titled "Estimating Walking Speed, Stride Length, and Stride Time using a Passive Floor Based Electronic Scavenging System", 
in the IEEE Transactions in Sensors Applications Symposium (SAS) in 2017 [4], and presented on March 15 in Glassboro, New Jersey, USA. Since this was our first thesis effort, we have made it chapter 3. We also submitted an abstract to Alzheimer's Association International Conference (AAIC2017), in UK. It was accepted and will be presented in July 2017. We have developed additional work on gait assessment, which is covered in a paper titled" Floor Based Sensor System: Additional Intelligence, Gait Estimation, and Scavenging Charging Characteristics", that provides statistics and correlation between the GAITRite mat and smart carpet systems. We submitted this paper to ICOMP'17 - The 18th International Conference on Internet Computing and Internet of Things.

We studied the characteristics of the scavenged signal from the smart carpet sensor. We investigated the characteristics of the sensor's accumulated charge or voltage level, measured the analog features like signal power, pule width, number of peaks, etc. We further, extracted computational features using Mel Frequency Cepstral Coefficients technique. This is in chapter 4. Initial work the scavenged signal analog features were submitted to ICOMP'17 conference.

We extended the capabilities of the smart carpet to count the plurality of people walking on it. We developed algorithms to, reliably, count the number of people walking on the carpet and evaluated the performance of the algorithms on a dataset collected from volunteers performed different walking scenario. We produced a paper titled "Counting Multiple People on a Floor Based Array sensor system". We submitted this paper to HIMS'17 - The 3rd International Conference on Health Informatics and Medical Systems, and made up chapter 5. 
We also extended our previous work from the Master's project on fall detection; this included developing and evaluating algorithms for detecting falls with more satisfactory sensitivity and specify [submitted to ICOMP'17]. We Evaluated algorithms on a data set collected by volunteers performed the walk, and falls in controlled lab settings. We submitted this work to ICOMP'17 conference.

The majority of the dissertation is dedicated to elucidating these objectives. We have adopted the practice requested by the committee to use our papers to describe our progress. We have also continued to improve our work and one chapter (4) describes yet unpublished work. 


\section{CHAPTER 2: BACKGROUND}

\subsection{Rationale}

Research focused on older adults continues to promote successful aging, especially regarding how to enhance the overall quality of life and provide adequate medical care while keeping health care cost under control. Technology is a welcome addition to the population of the elderly; it offers the elderly productive and independent lives [7]. An obvious goal, which we understood, was to develop new technology or enhance existing ones to detect falls and so help reduce the consequences of a fall $[2,3]$. All fall detection systems have a common objective of distinguishing a fall from activities of daily living, which tends not to be an easy problem to solve. Fall prediction or fall risk analysis extends the functionality of smart carpet. Fall risk can be assessed by extracting and estimating Gait Parameters [8]. Recent research shows that change in gait parameters may be predictive of future falls and adverse events in older adults such as physical functional decline [9-12] and fall risks [13,14].

Many factors affected the choice of the ideal system; the cost, portability, and active involvement from the user. Many systems are suitable only for lab or clinic settings, e.g. GAITRite Electronic mat, and the Vicon motion capture system. Therefore, there is a need to have systems that continuously measure and report gait parameters during every day activities outside labs. The drawback of lab specific systems is that the individuals feel instructed to walk in a certain way, in other words, not in their natural daily activity that reflects their actual gait behavior. Many technologies have been 
studied, developed and enhanced to be an alternative to the expensive and lab controlled systems.

Wearable sensors systems that consist of accelerometer and /or gyroscopes to measure gait parameters have been proposed [15-18]. Researchers used different setups, number of sensors, and derived a variety of parameters to assess individuals' gait, and fall risks with good agreements compared to gold standards. Some of the of wearable's drawbacks are the need to have the individuals worry about charging, wearing or taking off the sensor, and transferring data from the sensor devices. The position of the sensor in smart phone, for example, is important to achieve good measurement; this will not be possible by simply having them in pockets or hand. People with dementia have limited ability to maintain and use such wearable devices. Studies have shown older adults to prefer non-wearable sensors [19] for in-home monitoring.

The Eldercare technology research group developed and evaluated different systems and technologies and validated their work with GAITRite, and Vicon motion capture systems. A low-cost, environmentally mounted, automated monitoring system based on the Microsoft Kinect has been developed that continuously monitors and reports the gait during normal everyday activities of elderly [20-26]. With its good results and ability to detect falls, assess gait, and other useful daily living functions, it may suffer degraded performance with occlusion, and limited depth range. Floor based sensors which measure the forces that are applied on the floor are widely spread. [27-29]. In [28] the investigators capture the time varying signal to measure weight distribution within certain areas. It requires fixed installation under the metal support structure for the 
sensors and floor tiles. In [29] they measured the ground reaction force of the user's foot by load cells to generate user identification.

\subsection{Smart Carpet}

Our lab uses context-aware, non-computer-vision based human recognition and fall detection system. It is a floor based array sensors system, i.e. smart carpet $[2,3]$, which in obtrusive and preserve privacy. One installs it in the home or apartment and additionally has usefulness in places where traditional sensing system might suffer complications like occlusion. The smart carpet system includes the sensor data acquisition, data manipulating, data reading, storage, display, and communication. The system operates by detecting the person's movement and storing the floor sensor data. The motion on the carpet activates a set of sensors that outputs a voltage signal. The system amplifies the signal, digitizes it, and then translates it with all other bits addressed in a single scan into a frame for further processing. We ran computational intelligence algorithms to detect falls [3] and now to measure and estimate people's gait. Further, our goal here is to accurately recognize, count and monitor the movements of the individuals walking on the smart carpet system.

Continuous tracking and monitoring residents who live alone is important, as some elderly are reluctant to live in nursing homes. These residents may have visitors, which motivates us to expand the functionalities of the smart carpet to be able to detect visitors. We developed algorithms to count the plurality of people by identifying the subgroups formed by the activated sensors when an individual walks on the carpet.

Looking at the problem from different perspective motivated our study of the sensor scavenged signal characteristics to relate to the residents or visitors. We 
constructed a new sensor segment sufficiently large to capture the variability of the signal characteristics, from which we can find power, pulse width, number of peaks, etc. To study the behavior of the smaller sensors we requested a sensor large enough to fit one full foot. Our initial analysis and results showed that signal analog characteristics did not provide discriminatory features, and are sensitive to a variety of conditions, like weather, shoe types, etc. We reevaluated the signal, by extracting more features applying the Mel frequency Cepstral Coefficient (MFCC) as described in [30, 31]. MFCC is useful in many applications such as voice recognition, satellite image identification, face recognition, etc. We are interested in this using MFCC technique to extract features from our 1D sensor signal. This allowed us to discriminate among walkers.

The extension of the smart carpet system demonstrated here will improve its utility and make the 24/7 monitoring and subsequent storage a valuable useful commodity. One can envision a future in which monthly evaluation of the smart carpet data will provide changes in gait, record of resident activity and a record of sociability. 


\section{CHAPTER 3: GAIT ESTIMATION}

\subsection{Introduction}

With this material, we produced a paper titled "Estimating Walking Speed, Stride Length, and Stride Time using a Passive Floor Based Electronic Scavenging System". The paper was published in the IEEE Transactions in Sensors Applications Symposium (SAS) in 2017 [4], and presented on March 15 in Glassboro, New Jersey, USA. We described the development and evaluation of algorithms to measure and estimate gait parameters of walking speed, stride time, and stride length, in a controlled lab settings using the smart carpet, and the GAITRite mat. Experiments with 9 participants were conducted on the smart carpet segments and validated by the GAITRite mat that was laid underneath the carpet. Good agreement between the systems was found. We compared our results to previous work that used existing systems like Kinect, Vicon, and web-camera, and found to be comparable in performance $[23,32-33]$. 


\title{
3.2 Manuscript: Estimating Walking Speed, Stride Length, and Stride Time using a Passive Floor Based Electronic Scavenging System
}

\author{
Fadi Muheidat ${ }^{1}$, Harry W. Tyrer ${ }^{1}$, Mihail Popescu ${ }^{2}$, Marilyn Rantz ${ }^{3}$ \\ ${ }^{I}$ Electrical and Computer Engineering department \\ ${ }^{2}$ Health Management and Informatics Department \\ ${ }^{3}$ Sinclair School of Nursing University of Missouri, Columbia, USA
}

\begin{abstract}
- we have developed a floor based personnel detection system to extract gait parameters including walking speed, stride length and stride time, we call it smart carpet. These parameters are validated with a GAITRite Electronic mat. The smart carpet is laid over the GAITRite mat, and subjects walked across the mat for 9 trials each. The data acquisition system of the smart carpet recorded the location of the active sensors, which were later used to extract the gait parameters. An excellent agreement for walking speed, stride length and stride time between the two systems is achieved. The mean percentage error difference for walking speed is $1.43 \%$, stride length is $-4.32 \%$ and stride time is $5.73 \%$. For walking speed, the standard deviation is 4.39 , which emphasizes that $68 \%$ of the error is within a $5 \%$. We compared our work to the work done by a research group who used a vision based Kinect and web cameras system with excellent agreement.
\end{abstract}

\section{$3.2 \nmid 1$.Introduction}

Clinical research has identified clear links between human gait characteristics and different medical conditions [1]. Recent research shows that change in certain gait parameters may be predictive of future falls and adverse events in older adults such as physical functional decline [2-5] and fall risks [6-8]. We describe a system that is unobtrusive and continuously monitors the gait during daily activities of elderly people. 
The early detection of gait decline will benefit the senior by providing an assessment of the risk of falls.

A variety of technologies are being developed and investigated to assess and estimate the gait parameters. Most of the systems can be categorized into wearable devices, walk-on devices, radar and motion systems, and vision based devices and techniques [9-14]. Many factors affected the choice of the ideal system; the cost, portability, and active involvement from the user. Many systems are suitable only for lab or clinic settings, e.g. GAITRite Electronic mat, and the Vicon motion capture system.

The Eldercare technology research group developed and evaluated different systems and technologies and validated their work with GAITRite, and Vicon motion capture systems. A low-cost, environmentally mounted, automated monitoring system based on the Microsoft Kinect has been developed that continuously monitors and reports the gait during normal everyday activities of elderly [13].

In this research, we focus on state of the art floor based personnel detector system we call the smart carpet. The system uses a signal-scavenging technique wherein a sensor made from a conductive material picks up stray $60 \mathrm{~Hz}$ noise to detect presence of the person. It has sensors installed under the carpet, and the electronics can send sensor activation data, which is modified to produce notifications to cell phones or email through the Internet when a fall or any set of events occur. Our laboratory developed a low cost system to detect human falls on the floor [15-18]. We built new carpet segments with new grounding layout to eliminate the effect of noise on the data collected. The smart carpet has the advantages of unobtrusive, no privacy issues, and relatively 
inexpensive. We now want to continuously measure the gait parameters during the daily activities of residents.

This paper presents a methodology for and results from estimating the gait using a floor based personnel detector system, and validating the system against the GAITRite electronic mat. Section II of this paper provides an overview of the system, and our methodology. Section III contains the experimental results and compared to the GAITRite. Finally, Section IV covers discussion, conclusion and future work.

\section{2\2. Methodology}

\section{a. System Overview}

The personnel detector was used to measure the gait. We call it the smart carpet and it is a floor based personnel detector system. The system consists of two segments each with a 32, 6"x 6", sensors separated by 6" space with a total carpet length of 16 feet. The sensors are connected to data acquisition system that scans at speed of 14 frames per second. Signals converted to digital values with a constant threshold of 600/1023 (10-bit Analog to Digital convertor), and then read by a computer as an ASCII frame of 10 bytes

length. Our software components parse the string and run computational algorithms to measure the gait. Figure 3-1 shows the full system setup. 


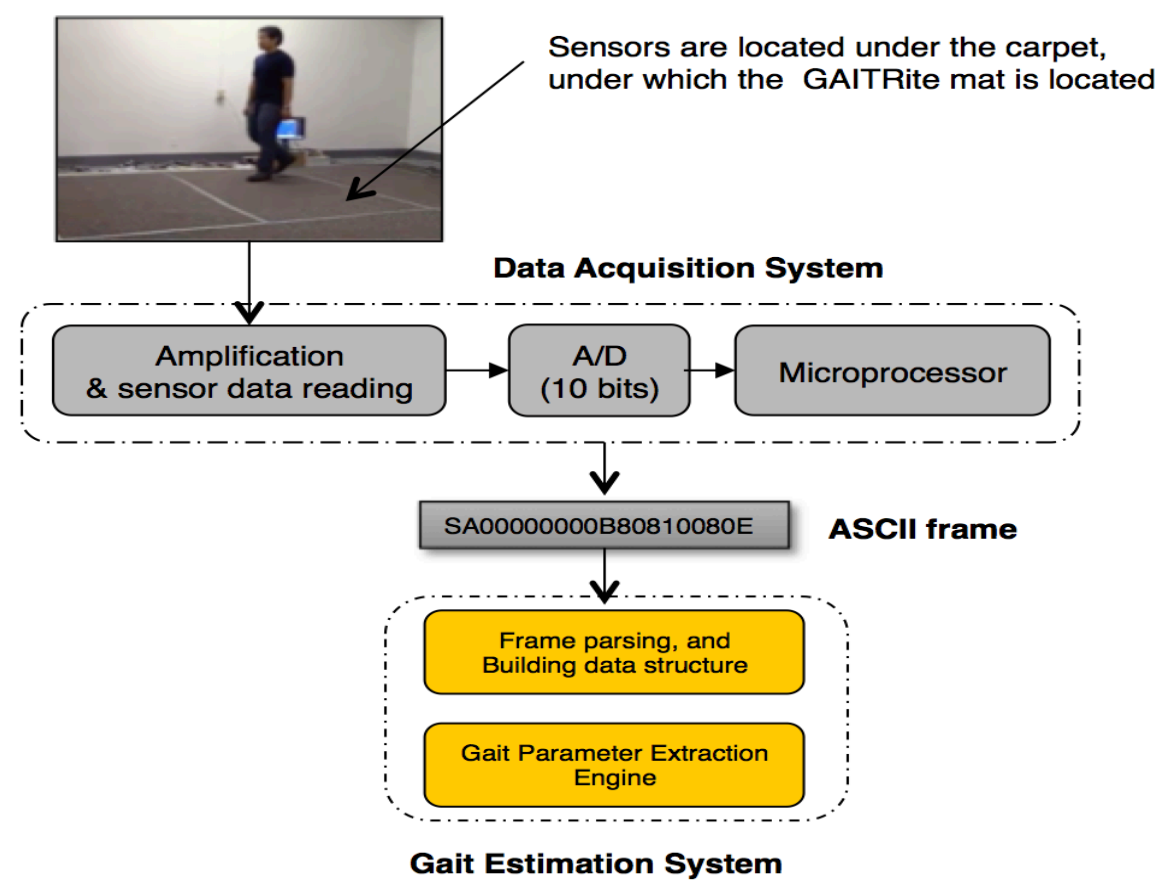

Figure 3-1 The full system developed. Sensors, data acquisition system and software components.

The GAITRite system [9] available commercially from CIR Systems Inc. and the gold standard for Temporospatial Gait Analysis was used to validate our method. The GAITRite System is an electronic walkway; we used it to measure the temporal (timing) and spatial (two-dimension geometric position) parameters by pressure activating its sensors during a walk. The GAITRite has an effective length of 16 feet $(4.9 \mathrm{~m})$. It comprises a series of sensor pads inserted in a grid, placed 0.5 inches $(1.27 \mathrm{~cm})$ apart (total of 18,432 sensors). Footfall data from the activated sensors are collected by a series of on-board processors and transferred to the computer through a serial port. The sampling rate of the system is $120 \mathrm{~Hz}$. The gait parameter definitions used in the GAITRite ${ }^{\circledR}$ instrumented walkway system are listed below: Ambulation time is defined as the time elapsed between first contact of one foot and the last footfall. Walking speed: Distance traveled divided by the ambulation time. Step time: Time elapsed from first 
contact of one foot to the first contact of the opposite foot. The step length of the right foot is defined as the distance between the centers of the left foot to the center of the right foot along the line of progression. Stride time: Time between successive footfalls of the same foot. In addition, the spatial gait parameter of Stride length is the distance between successive footfalls of the same foot.

\section{b. Mesuring Gait Parameters}

In this section, the techniques used to estimate the gait parameters of walking speed, stride time, and stride length from the smart carpet are described. The parameters we measured are distance travelled, and elapsed time. We used computational and heuristics rules to determine the number of footfalls; we used these to calculate the walking speed, stride time and length.

\section{i. Foot Falls}

In addition to the walk time and distance, we found that determining the footfalls is a key factor in measuring and calculating gait parameters. We determined the footfalls by grouping the scanned frames in a window of size $\mathrm{W}_{\mathrm{N}}$, and then counted the number of active sensors. We set a threshold $(\mathrm{TH})$, and sensors count greater than that value we considered a valid footfall. We found that the window size $\mathrm{W}_{\mathrm{N}}$ is determined based on the walking speed at constant scanning rate (14 frames per second). A subject performs two walk sequences to find the average speed (distance/time), and then we determine the proper window size $\mathrm{W}_{\mathrm{N}}$ to determine the footfall.

\section{ii. $\quad$ Walking Speed}


We locate the first and last active sensors on the smart carpet from which we determine the distance walked and the time elapsed. Walking speed $\boldsymbol{V}$ can be calculated by dividing the distance by the time according the equation:

$$
V=\frac{D}{T}
$$

$V$ : walking Speed

$D$ : Distance Travelled, and

T: Time Elapsed

\section{iii. $\quad$ Step and Stride Time}

Step time, which is the time elapsed from first contact of one foot to the first contact of the opposite foot, is computed by dividing the Time Elapsed $(\boldsymbol{T})$ by the number of Steps, $\boldsymbol{N}_{\text {steps }}($ Footfalls -1$)$ as in

$$
T_{\text {step }}=T / N_{\text {steps }}
$$

We use this time to compute the Stride Time:

$$
\boldsymbol{T}_{\text {stride }}=\mathbf{2} * \boldsymbol{T}_{\text {step }}
$$

\section{iv. $\quad$ Step and Stride Length}

Step length $\boldsymbol{L}_{\text {step }}$ is measured by dividing the distance travelled by the number of steps.

$$
L_{\text {step }}=D / N_{\text {steps }}
$$

Similarly, the stride length is computed by doubling the step length. 


$$
L_{\text {stride }}=2 * L_{\text {step }}
$$

We did not distinguish the left and right foot even though we believe we can find the centroid of each footfall to determine the location of left and right footsteps. To obtain the gait parameters we developed algorithm I.

We first read a frame of data (corresponding to one scan of the carpet segments), and then converted into binary to build matrix of "0" and "1", where "1" represents an active sensor. We then placed the frames in a group of lengths $\mathrm{W}_{\mathrm{N}}$, and then find the total count of active sensors, which if within a certain threshold defined experimentally will be marked as footfall.

In rare occasions we have bad walks, where we were unable to detect a footfall in the middle of the walk. This affected our calculations, and we dropped this bad walk trial. However, if we could detect footfalls at the middle of the walk range but not detect the footfall at the beginning or the end of the walk trial, we still considered the walk valid, since we had several valid steps. In this case the number of steps, time elapsed, and distance travelled will be less than measured in GAITRite, but this will not affect computing the walking speed, stride length and stride time. 


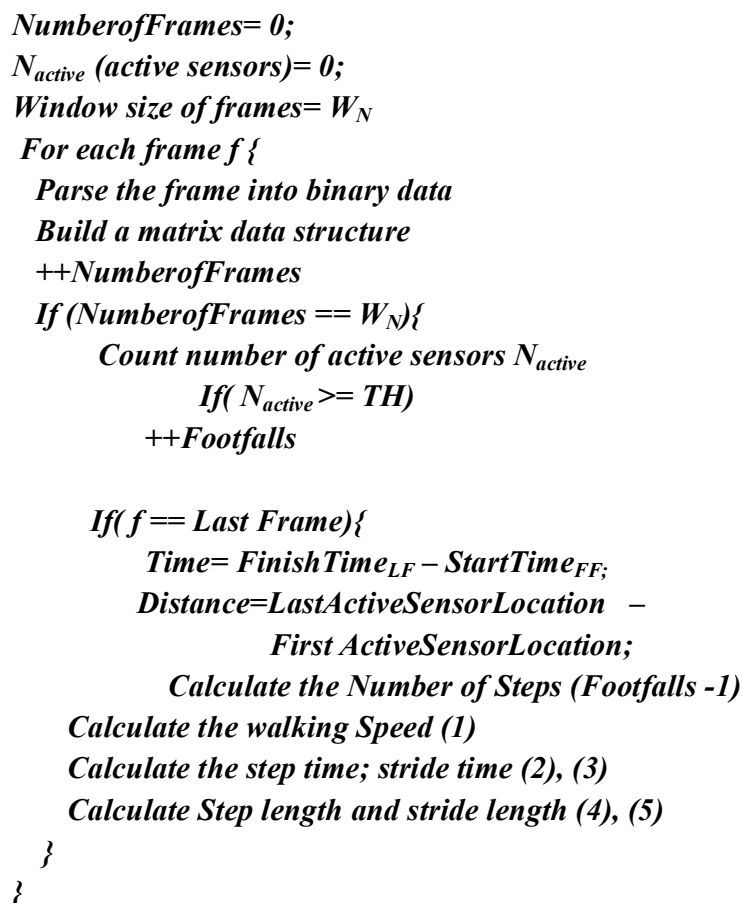

\section{c. Subjects and Repeated Trials}

We placed the two-segment carpet at the top of the GAITRite mat aligned to the active region of the GAITRite mat, and the subjects walked across the carpet. The walk data are recorded in raw file and later used to extract the gait parameters.

Nine subjects participated in the experiment. Each subject was tested multiple times, trying to maintain same walk pace. In total, there are 75 walk sequences. Subjects normally take about 5-10 steps to complete the walkway. As indicated previously, a few (5 out of 80 ) walk sequences were excluded due to noisy sensors that produced clearly abnormal results. 


\section{2\3. Experimental Results}

Gait parameters were extracted and computed after collecting 75 walk sequences from the smart carpet and GAITRite systems. Figure 3-2 shows plots of walking speed, stride time, and stride length for each of the walking sequences as computed for both systems. The walking speeds obtained from the tests cover a large variation ranging from around $26.1 \mathrm{in} / \mathrm{sec}(66.3 \mathrm{~cm} / \mathrm{sec})$ to $53.31 \mathrm{in} / \mathrm{sec}(135.4 \mathrm{~cm} / \mathrm{sec})$.

We computed the percentage differences between our system, the smart carpet, and the GAITRite system for walking speed, stride time and stride length. Table I. shows the percentage difference mean and standard deviation of walking speed, stride time, and stride length for each of the walking sequences as computed for both systems. For walking speed, the mean of $1.43 \%$ is certainly within a $5 \%$ error that might be expected. The standard deviation is $4.39 \%$, which emphasizes that $68 \%$ of the error is within a $5 \%$.

We used the GAITRite as the gold standard; measurements were obtained and used to calculate the average stride length, using both feet of the subject performing the walk. In contrast, the average stride length for the smart carpet system is calculated using equation 5 . We also computed the stride length by multiplying the walking speed by the stride time $(2 *$ step time). There was no significant difference between the two methods for the smart carpet measures. The GAITRite and smart carpet data showed little difference, specifically, mean percentage difference and standard deviation were $0.16 \%$ and $0.66 \%$ respectively.

Experimental results show that for all subjects, the step time is longer than 0.5 seconds independently of the walking speed. Stride time (double the step time) ranges from $1.08 \mathrm{sec}$ to $1.76 \mathrm{sec}$. The percentage difference is large $24 \%$ although the difference 
is only $0.3 \mathrm{sec}$. Results from Table I show close agreement between the stride length and stride time error percentages
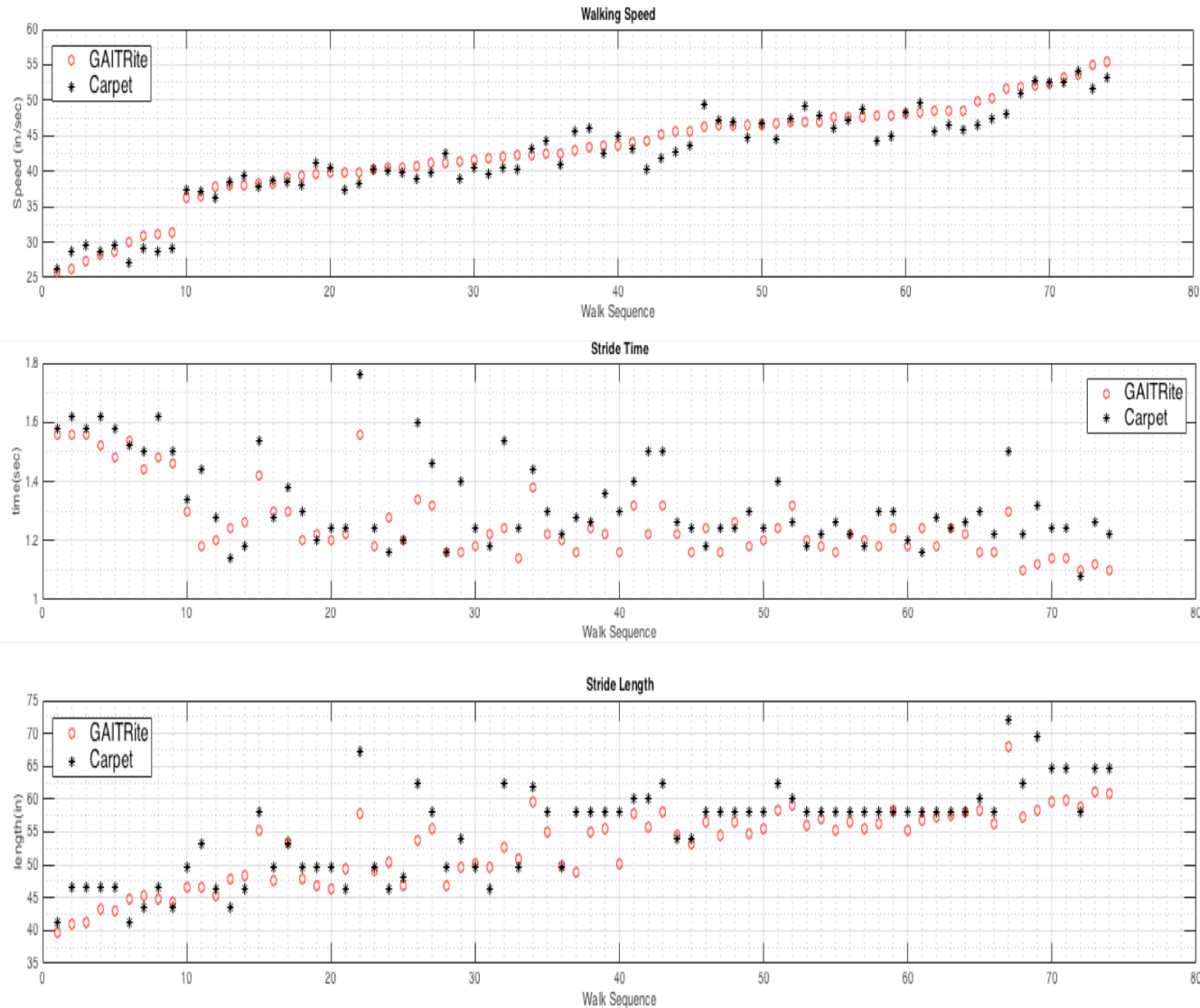

Figure 3-2. Walking Speed, Stride Time, and Stride Length: Smart Carpet vs GAITRite Nine subjects walk with repetition results comparing walking speed from Smart Carpet and GAITRite systems. Walks sorted in ascending order of speed.

TABLe I. Difference in WALKIng SPEed, STRIDE TIME AND LENGTH

\begin{tabular}{|l|c|c|c|}
\hline & Walking Speed & Stride Time & Stride Length \\
\hline Mean \% Diff & 1.43 & -5.73 & -4.32 \\
\hline Std. Deviation & 4.39 & 7.19 & 6.04 \\
\hline
\end{tabular}




\section{2 $\backslash$ 4.Discussion and conclusion}

Gait parameters extracted from the smart carpet achieved an excellent agreement compared to the GAITRite system. In a few walk trials we had errors; that is a difference from the average value of the parameters for each system. For example; for walking speed of one trial, the percentage difference was the highest error of $10.1 \%$. In this walking sequence, the number of footfalls differed from the number of footfalls measured from GAITRite. This is a result of the way sensors were activated on the carpet. The smart carpet measured time 6.869 seconds which is bigger than the actual time on the GAITRite 5.96 seconds; the distance travelled on the carpet as measured by the active sensors locations was 15.5 foot $(472.44 \mathrm{~cm})$ is slightly higher than the one on the GAITRite 14.95 foot $(455.8 \mathrm{~cm})$. These differences we believe arise from the spatial sampling nature of our devices.

We compared our system with other systems developed by the Eldercare Technology research group: Kinect, and web cameras. They used as ground truth the GAITRite and the Vicon motion capture systems shown in TABLE II, TABLE III, and Table IV. The data has a high level of agreement. Data was collected from different systems and environments; lab settings, and smart home in Tiger place, different subjects and different trials.

TABLE II. \% DIFFERENCE IN WALKING SPEED

\begin{tabular}{|l|c|c|c|c|}
\hline & $\begin{array}{c}\text { Smart Carpet vs } \\
\text { GAITRite }\end{array}$ & $\begin{array}{c}\text { Kinect vs } \\
\text { Vicon [19] }\end{array}$ & $\begin{array}{c}\text { Kinect vs } \\
\text { Vicon [20] }\end{array}$ & $\begin{array}{c}\text { Web Camera vs } \\
\text { GAITRite [21] }\end{array}$ \\
\hline Mean \% Diff. & 1.43 & -2.1 & 2.9 & 0.18 \\
\hline Std. Deviation & 4.39 & 1.1 & 1.7 & 2.32 \\
\hline
\end{tabular}


TABLE III. \% DIFFERENCE IN STRIDE TIME

\begin{tabular}{|l|c|c|c|c|}
\hline & $\begin{array}{c}\text { Smart Carpet vs } \\
\text { GAITRite }\end{array}$ & $\begin{array}{c}\text { Kinect vs } \\
\text { Vicon [19] }\end{array}$ & $\begin{array}{c}\text { Kinect vs } \\
\text { Vicon [20] }\end{array}$ & $\begin{array}{c}\text { Web Camera vs } \\
\text { GAITRite [21] }\end{array}$ \\
\hline Mean \% Diff. & -5.73 & -0.1 & 0.6 & -0.63 \\
\hline Std. Deviation & 7.19 & 2.4 & 1.5 & 2.46 \\
\hline
\end{tabular}

TABLE IV \% DIFFERENCE IN STRIDE LENGTH

\begin{tabular}{|l|c|c|c|c|}
\hline & $\begin{array}{c}\text { Smart Carpet vs } \\
\text { GAITRite }\end{array}$ & $\begin{array}{c}\text { Kinect vs } \\
\text { Vicon [19] }\end{array}$ & $\begin{array}{c}\text { Kinect vs } \\
\text { Vicon [20] }\end{array}$ & $\begin{array}{c}\text { Web Camera vs } \\
\text { GAITRite [21] }\end{array}$ \\
\hline Mean \% Diff. & -4.32 & -0.7 & 5.3 & -0.71 \\
\hline Std. Deviation & 6.04 & 2.7 & 1.9 & 3.04 \\
\hline
\end{tabular}

The floor based personnel passive detector system was able to measure and extract gait parameters. Enhancing the footfall detection, and measuring accurate travelled distance will yield better matching and reduced percentage difference compared to GAITRite and Vicon motion capture system.

Future work will look into further developing algorithms to detect the footfalls. In addition, we can use connected component labeling algorithm to have better footfall detection. Building high-resolution carpet by reducing the size of the physical sensor is possible. This will clearly increase the accuracy for detecting the footfall. It will not materially improve of the measured travelled distance or the other global variable we measure. It could some improvement in accuracy, but it may not be needed. 


\title{
Acknowledgment
}

We would like to thank professor Marjorie Skubic for advice and assistance. Our deep gratitude to Andrew Craver (Andy) from Tiger place for his support on using the

\author{
GAITRite mat and software.
}

\section{References}

[1] D. Hodgins, "The Importance of Measuring Human Gait," Medical Device Technology. 2008 Sep;19(5):42, 44-7.

[2] Viccaro LJ, Perera S, Studenski SA. Is timed up and go better than gait speed in predicting health, function, and falls in older adults? J Am Geriatr Soc. 2011;59(5):887 92.

[3] Peel NM, Kuys SS, Klein K. Gait speed as a measure in geriatric assessment in clinical settings: a systematic review. J Gerontol Ser A Biol Sci Med Sci. 2013;68(1):39-46.

[4] Van Kan GA, Rolland Y, Andrieu S, Bauer J, Beauchet O, Bonnefoy M, Cesari M, Donini L, GilletteGuyonnet S, Inzitari M, et al. Gait speed at usual pace as a predictor of adverse outcomes in community-dwelling older people an international academy on nutrition and aging (iana) task force. J Nutr Health Aging. 2009;13(10):881-9.

[5] Rochat S, Büla CJ, Martin E, Seematter-Bagnoud L, Karmaniola A, Aminian K, Piot-Ziegler C, Santos-Eggimann B. What is the relationship between fear of falling and gait in well-functioning older persons aged 65 to 70 years? Arch Phys Med Rehabil. 2010;91(6):879-84.

[6] Rochat S, Büla CJ, Martin E, Seematter-Bagnoud L, Karmaniola A, Aminian K, Piot-Ziegler C, Santos-Eggimann B. What is the relationship between fear of falling and gait in well-functioning older persons aged 65 to 70 years? Arch Phys Med Rehabil. 2010;91(6):879-84.

[7] Taylor ME, Ketels MM, Delbaere K, Lord SR, Mikolaizak AS, Close JC. Gait impairment and falls in cognitively impaired older adults: an explanatory model of sensorimotor and neuropsychological mediators. Age Ageing. 2012;41(5):665-9.

[8] Stone EE, Skubic M. Capturing habitual, in-home gait parameter trends using an inexpensive depth camera. In: Engineering in Medicine and Biology Society (EMBC), 2012 Annual International Conference of the IEEE. IEEE; 2012. p. 5106-9. doi:10.1109/EMBC.2012.6347142.

[9] K. E. Webster, J. E. Wittwer, Julian F. Feller, "Validity of GAITRite Walkway System for the Measurement of Averaged and Individual Step Parameters of Gait", Gait and Posture 22, pp 317-321, 2005.

[10] M. Skubic, G. Alexander, M. Popescu, M. Rantz, and J. Keller, "A Home Application to Eldercare: Current Status and Lessons Learned," Technology and Health Care, in press.

[11] F. Wang, E. Stone, W. Dai, M. Skubic and J. Keller, "Gait analysis and validation using voxel data," 2009 Annual International Conference of the IEEE Engineering in Medicine and Biology Society, Minneapolis, MN, 2009, pp. 6127-6130. doi: 10.1109/IEMBS.2009.5334706

[12] L. Liu, M. Popescu, M. Skubic and M. Rantz, "An automatic fall detection framework using data fusion of Doppler radar and motion sensor network," $201436^{\text {th }}$ Annual International Conference of the IEEE Engineering in Medicine and Biology Society, Chicago, IL, 2014, pp. 5940-5943. doi: 10.1109/EMBC.2014.6944981

[13] E. E. Stone and M. Skubic, "Unobtrusive, Continuous, In-Home Gait Measurement Using the Microsoft Kinect," in IEEE Transactions on Biomedical Engineering, vol. 60, no. 10, pp. 2925-2932, Oct. 2013. doi: 10.1109/TBME.2013.2266341 
[14] Alwan, M.; Rajendran, P.J.; Kell, S.; Mack, D.; Dalal, S.; Wolfe, M.; Felder, R. A smart and passive floor-vibration based fall detector for elderly. In Proceedings of the Second Information and Communication Technologies, Damascus, Syria, 24-28 April 2006; pp. 1003-1007

[15] Neelgund R., "Floor sensor development using signal scavenging for personnel detection system", University of Missouri, Columbia 2010. Masters thesis for fulfillment of MS Degree.

[16] Chinchao Suriyakul," a new circuit for personnel detection using signal scavenging", University of Missouri, Columbia 2012. Masters thesis for fulfillment of MS Degree.

[17] Gadre K., "Fall detection system using low cost computing and online Communication", University of Missouri, Columbia 2012. Masters thesis for fulfillment of MS Degree.

[18] F. Muheidat and H. W. Tyrer, "Can we make a carpet smart enough to detect falls?," 201638 th Annual International Conference of the IEEE Engineering in Medicine and Biology Society (EMBC), Orlando, FL, USA, 2016, pp. 5356-5359. doi: 10.1109/EMBC.2016.7591937

[19] Erik Stone , Marjorie Skubic, Evaluation of an inexpensive depth camera for in home gait assessment, Journal of Ambient Intelligence and Smart Environments, v.3 n.4, p.349 361, December 2011. doi:10.3233/AIS-2011-0124

[20] E.Stone, "Unobtrusive, In-Home Gait Measurement, Fall Risk Assessment, and Fall Detection Using Depth Imagery," Ph.D. dissertation, University of Missouri, Columbia, MO, USA, Dec. 2013

[21] F.Wang, "Motion analysis for in-home gait and balance assessment using inexpensive video sensors," Ph.D. dissertation, University of Missouri, Columbia, MO, USA, Jul. 2011.

\subsection{Further publications}

We have developed additional work on gait assessment, which is covered in a paper titled" Floor Based Sensor System: Additional Intelligence, Gait Estimation, and Scavenging Charging Characteristics" [5], that provides statistics and correlation between the GAITRite mat and smart carpet systems. The paper was submitted to ICOMP'17- The 18th International Conference on Internet Computing and Internet of Things. This work is part of chapter 6 . We investigated whether the differences between the two systems are statistically significant. The Statistical T-Test showed excellent agreement between the smart carpet and the GAITRite in estimating gait parameters. Correlations were calculated between GAITRite and smart carpet data for three gait parameters; walking speed, stride time and stride length. The $r$-squared of 93\% for walking speed showed great agreement between the two systems. Similarly, we had agreement in stride length (80\%), and stride time (63\%). 


\subsection{Details and additional work not yet published}

\subsubsection{Preliminary experiments}

In order to evaluate the use of the smart carpet to measure and estimate gait parameters, our preliminary work used four people (two adults, and two children).

They performed a set of walks on a two 8-foot carpet segments laid over the

TABLE 3-5 THE AVERAGE OF THE GAIT PARAMETERS: WALKING SPEED, STEP, AND STRIDE LENGTH, STEP AND STRIDE TIMES PER SUBJECT

\begin{tabular}{|c|c|c|c|c|c|c|c|c|}
\hline \multirow[t]{2}{*}{ Gait Parameter } & \multicolumn{2}{|c|}{$\begin{array}{l}\text { Adult-Male } \\
\text { (200 lb, 5’9”) }\end{array}$} & \multicolumn{2}{|c|}{$\begin{array}{l}\text { Adult-Female } \\
\text { (150 lb, 5’3”) }\end{array}$} & \multicolumn{2}{|c|}{$\begin{array}{l}\text { Child-Female } \\
\text { (98 lb, 4'8”) }\end{array}$} & \multicolumn{2}{|c|}{$\begin{array}{l}\text { Child-Male } \\
\text { (49 lb, 3'11”) }\end{array}$} \\
\hline & SC & GR & SC & GR & SC & GR & SC & GR \\
\hline \#Steps & 7 & 6 & 8 & 8 & 7 & 6 & 9 & 8 \\
\hline Stride time (sec) & 1.3 & 1.26 & 1.5 & 1.34 & 1.15 & 1.08 & 1.02 & 0.94 \\
\hline Stride length $(\mathrm{cm})$ & 127 & 138.3 & 118.9 & 101 & 132.2 & 126.7 & 111 & 106.3 \\
\hline $\begin{array}{l}\text { Waking Speed } \\
(\mathrm{cm} / \mathrm{sec})\end{array}$ & 99.4 & 106.6 & 77.3 & 77.5 & 115 & 118 & 110 & 109.7 \\
\hline
\end{tabular}

SC: Smart Carpet, GR: GAITRite

GAITRite mat. Each subject performed 4 walk trials. Table 3.5 shows the average results of the gait parameters: walking speed, step, and stride length, step and stride times, and number of steps. Figure 3.3 shows the measurements of the individual walks performed by Subject 2 (adult-Female). We noticed that the good agreement between the two systems in the walking speed (bottom of figure 3.3). This result motivated us to proceed with more formal experiments previously described. Figure 3.4 plots the mean, and variances for the four subjects for walking speed, stride time and stride length. As results show, there 
were outliers. However, there was some variability in the data distributions for different walks. Subject 2, for example, has larger variability in the walking speed. Looking at the individual walk data we found that the subject changed walking pace. Subject 3 has changed step/stride length by walking same distance with longer steps.
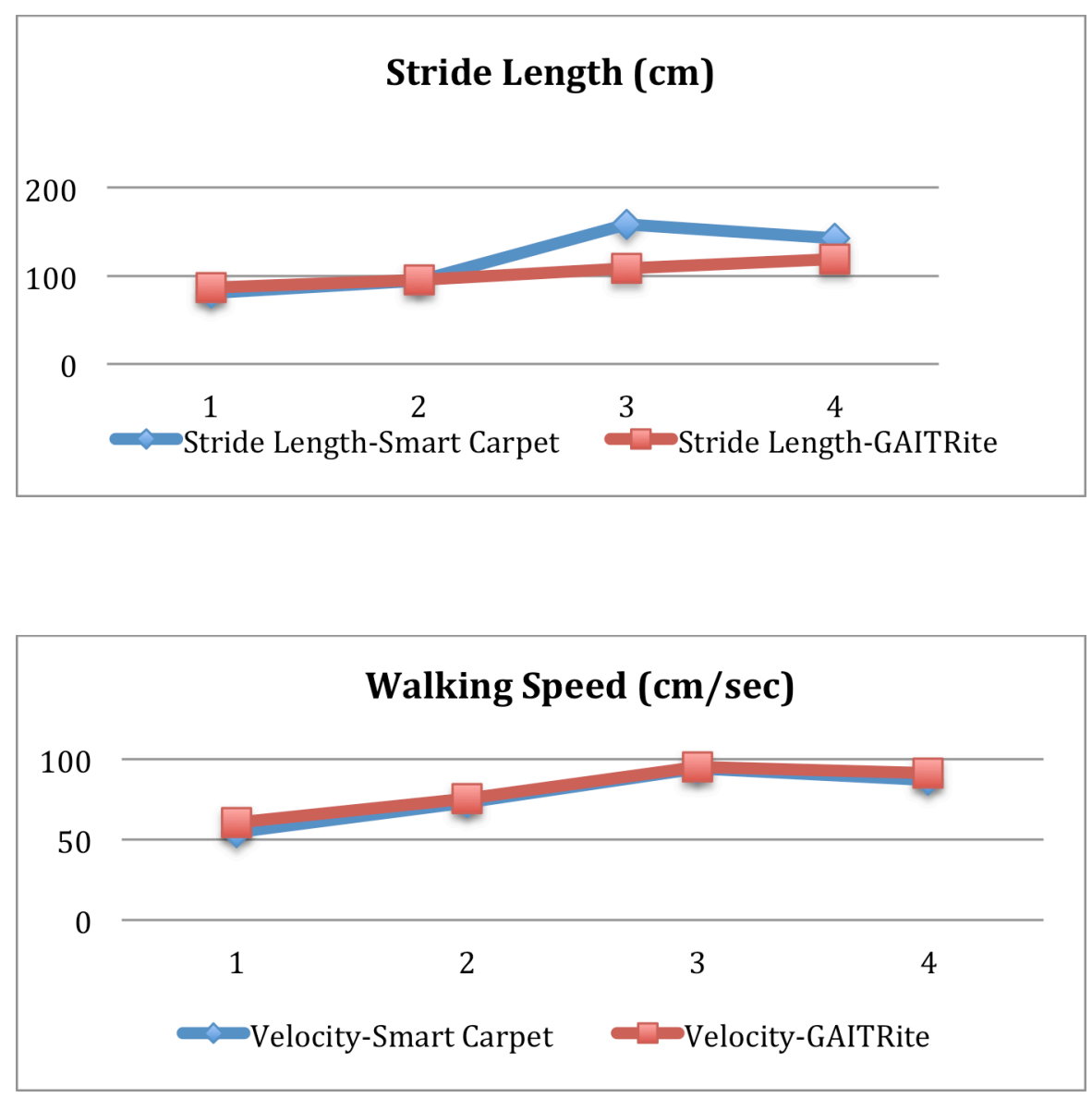

Figure 3.3 Stride length and Walking Speed for subject 2 for 4 different walk sequences. 

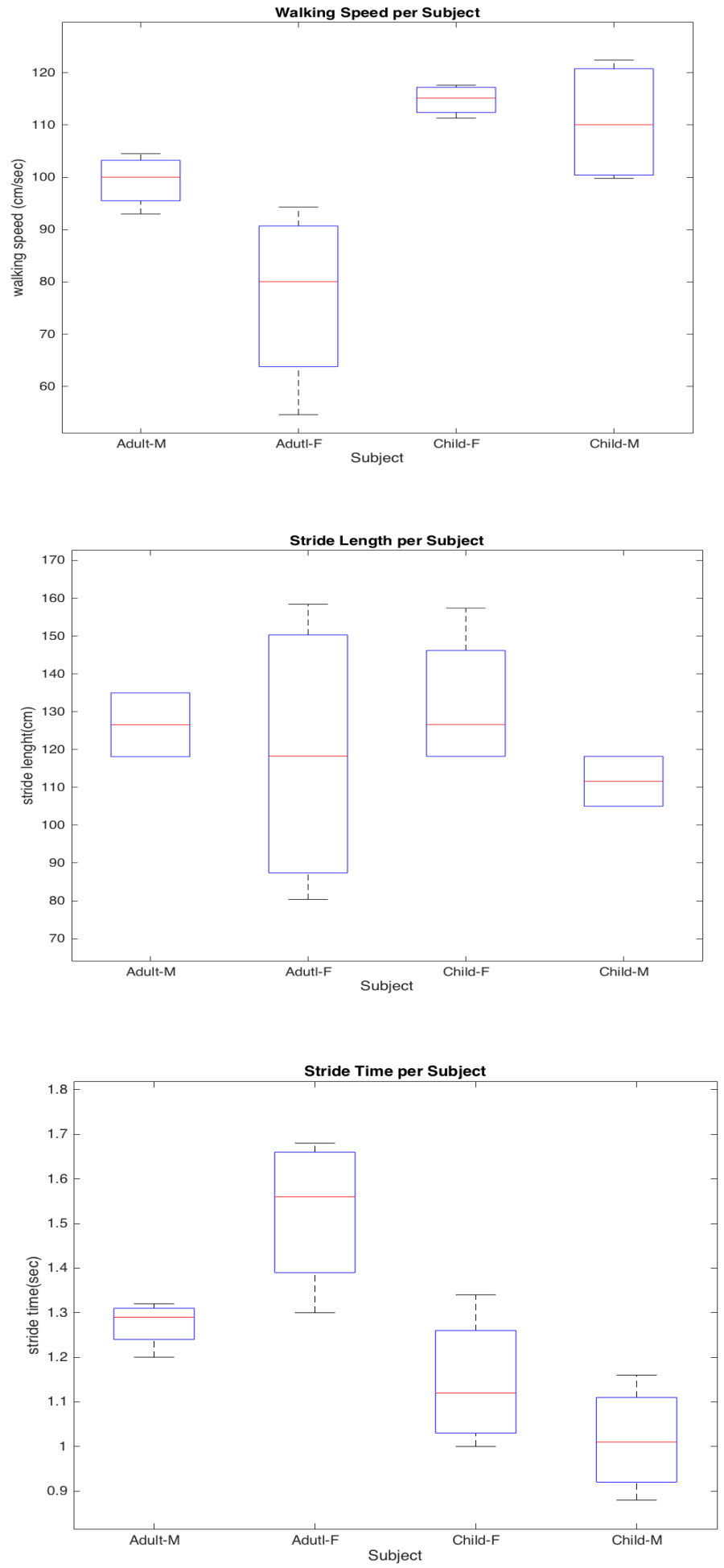

Figure 3.4 Box plot for Walking Speed, Stride Time and Length for four subjects 


\subsubsection{Subjects Results}

Nine subjects whose gait performance we documented previously, we further evaluated the smart carpet gait parameters data. Results presented in Table 3.6 show the average walking speed, stride length, and stride time for each subject. The subjects have different weight, height and walking speed. As shown in Table 3.6; subject 3 has the slowest walking speed, and shortest stride length, and most walking steps. During the experiments the volunteer tended to walk

Table 3.6 PER Subject Walking Speed, Stride Time, and Stride Length

\begin{tabular}{|l|l|l|l|l|l|l|l|}
\hline \multirow{2}{*}{} & \multicolumn{3}{|l}{ Subject 1 } & \multicolumn{2}{l}{ Subject 2 } & \multicolumn{2}{l|}{ Subject 3 } \\
\cline { 2 - 8 } & Carpet & G.Rite & Carpet & G.Rite & Carpet & G.Rite \\
\hline Walk Speed (cm/sec) & 103.34 & 101.5 & 131.78 & 134.58 & 72.33 & 73.26 \\
\hline Stride Time (sec) & 1.25 & 1.21 & 1.23 & 1.12 & 1.57 & 1.51 \\
\hline Stride Length (cm) & 128.66 & 119.91 & 161.04 & 149.86 & 113.51 & 109.41 \\
\hline & Subject 4 & & Subject 5 & \multicolumn{2}{l|}{ Subject 6 } \\
\hline & Carpet & G.Rite & Carpet & G.Rite & Carpet & G.Rite \\
\hline Walk Speed (cm/sec) & 102.93 & 105.07 & 118.2 & 120 & 120.18 & 121.49 \\
\hline Stride Time (sec) & 1.25 & 1.2 & 1.25 & 1.19 & 1.24 & 1.23 \\
\hline Stride Length (cm) & 127.89 & 127.07 & 147.32 & 142.28 & 148.45 & 145.58 \\
\hline & Subject 7 & & Subject 8 & & Subject 9 \\
\hline & Carpet & G.Rite & Carpet & G.Rite & Carpet & G.Rite \\
\hline Walk Speed (cm/sec) & 104.05 & 105.77 & 102.37 & 104.53 & 109.55 & 114.15 \\
\hline Stride Time (sec) & 1.44 & 1.28 & 1.25 & 1.21 & 1.5 & 1.35 \\
\hline Stride Length (cm) & 149 & 138.88 & 127.48 & 126.34 & 163.41 & 152.28 \\
\hline
\end{tabular}

slowly in short steps, which we believe was to be certain to make sure the individual was stepping within the allowed area of both the smart carpet and the GAITRite mat active region. Subject 2 walked a little bit faster than the rest of 
subjects, as he tended to be more relaxed and confident while performing all walk sequences. Both subjects 2 and 9 have the longest stride length, the same average number of steps ( 5 steps), but different walking speeds. Subject 9 is taller and took him longer time to finish the walk. The result in the table shows the average of the trials performed by the subjects. However the variability among the individual walks is shown in Figure 3.5. The Box plot shows the mean, range, and outliers.

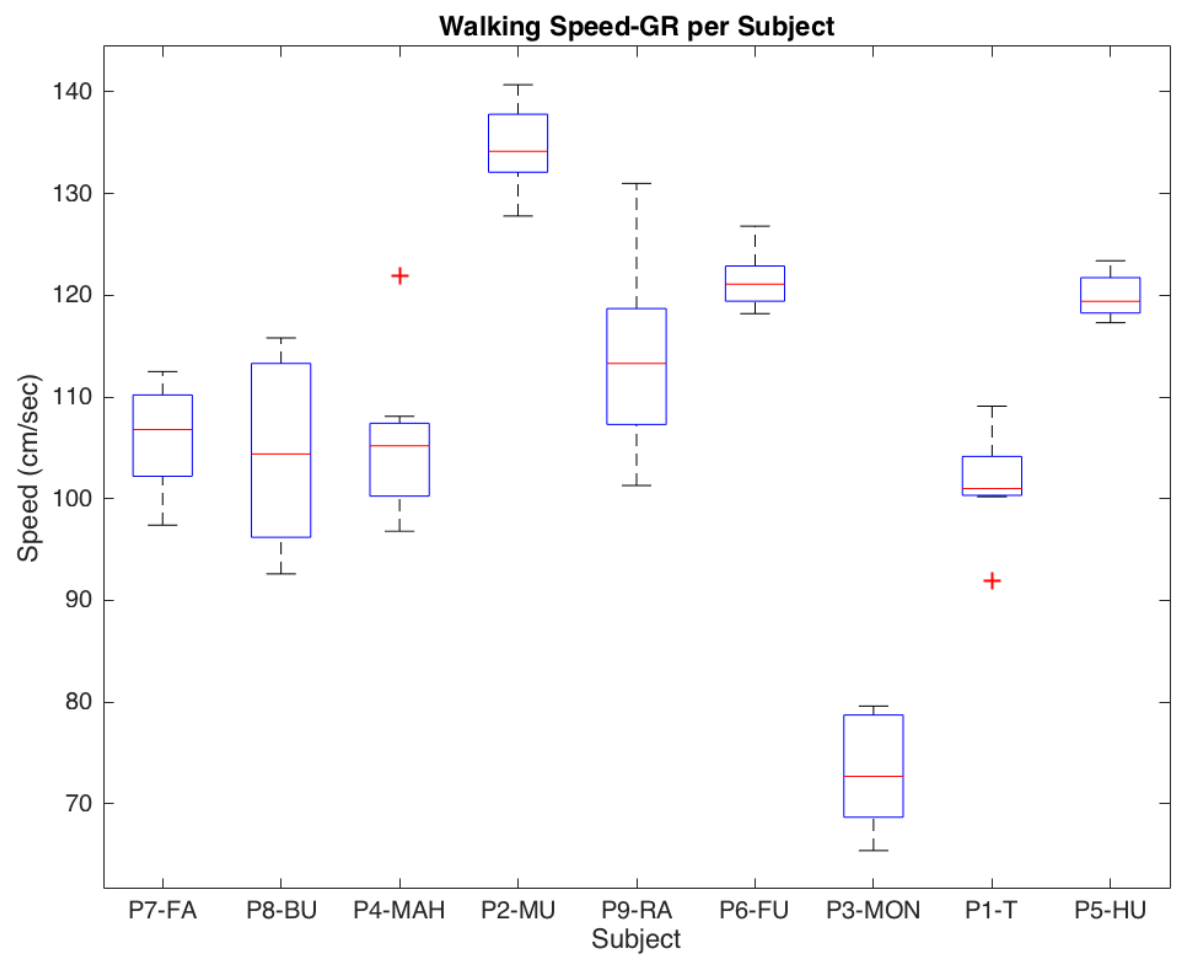

(a) 


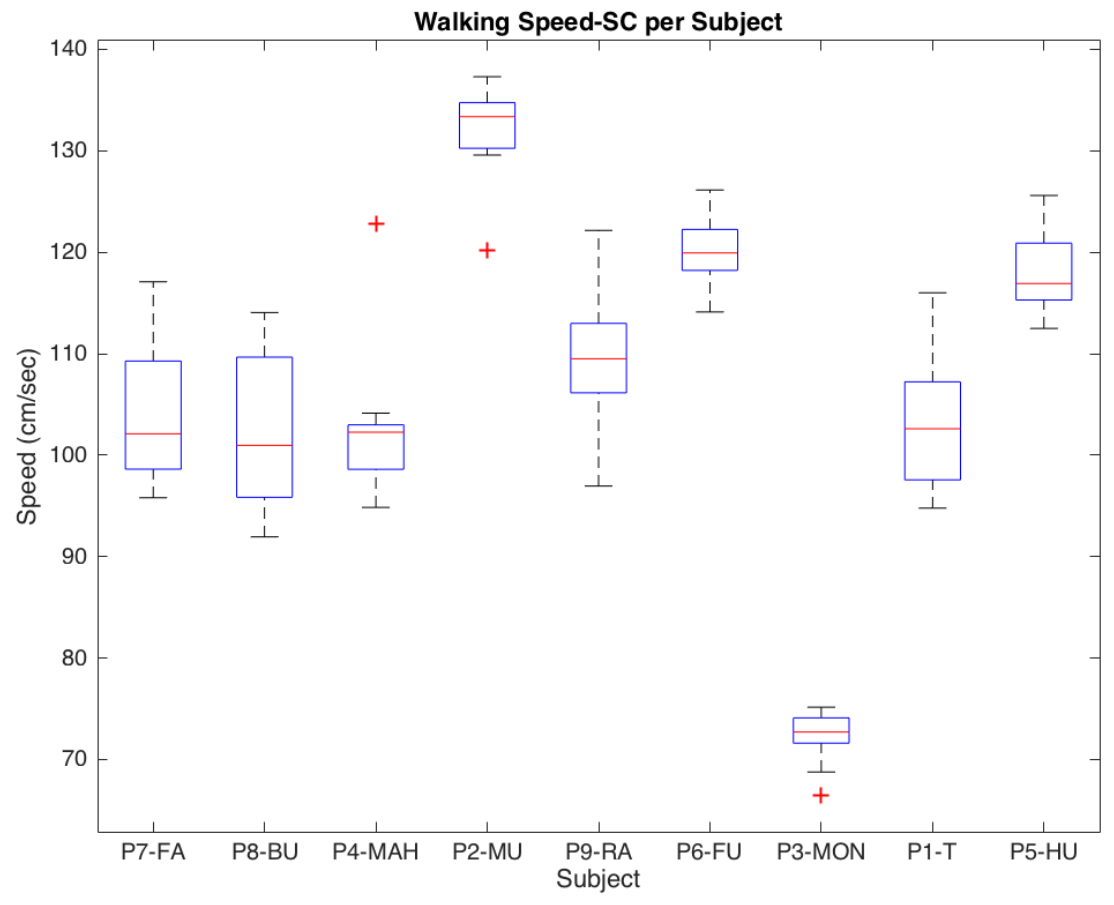

(b) 


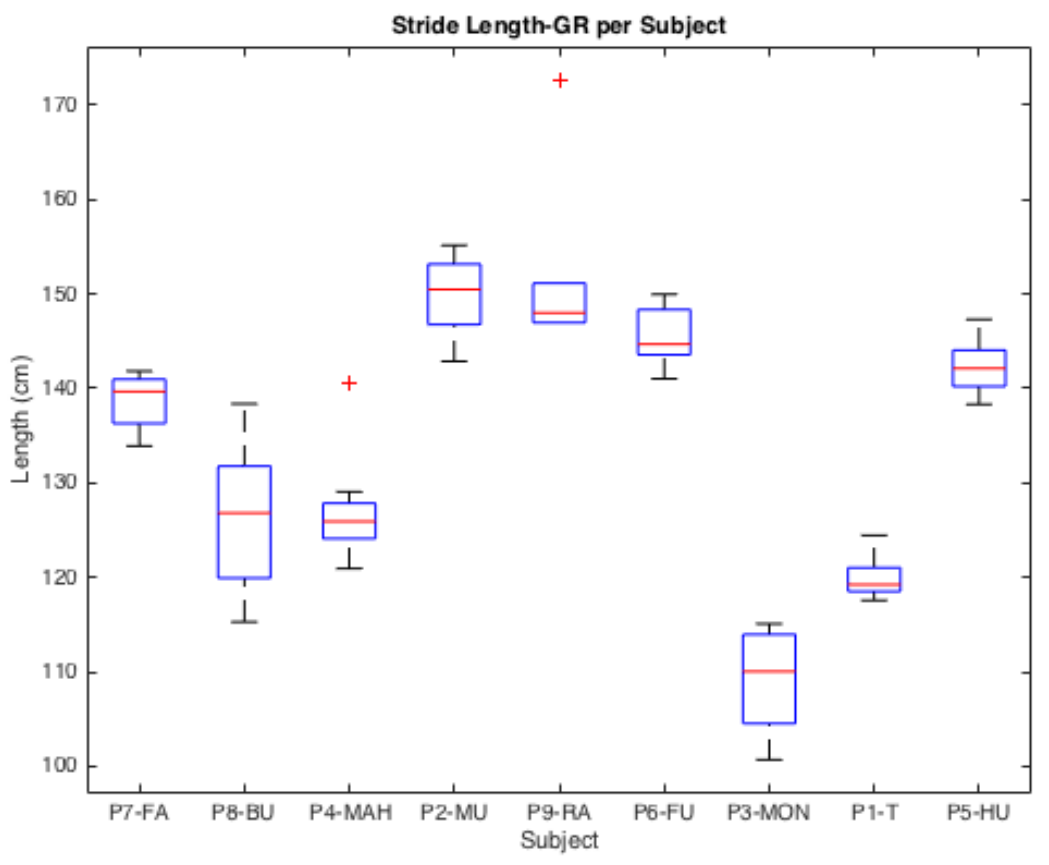

(c)

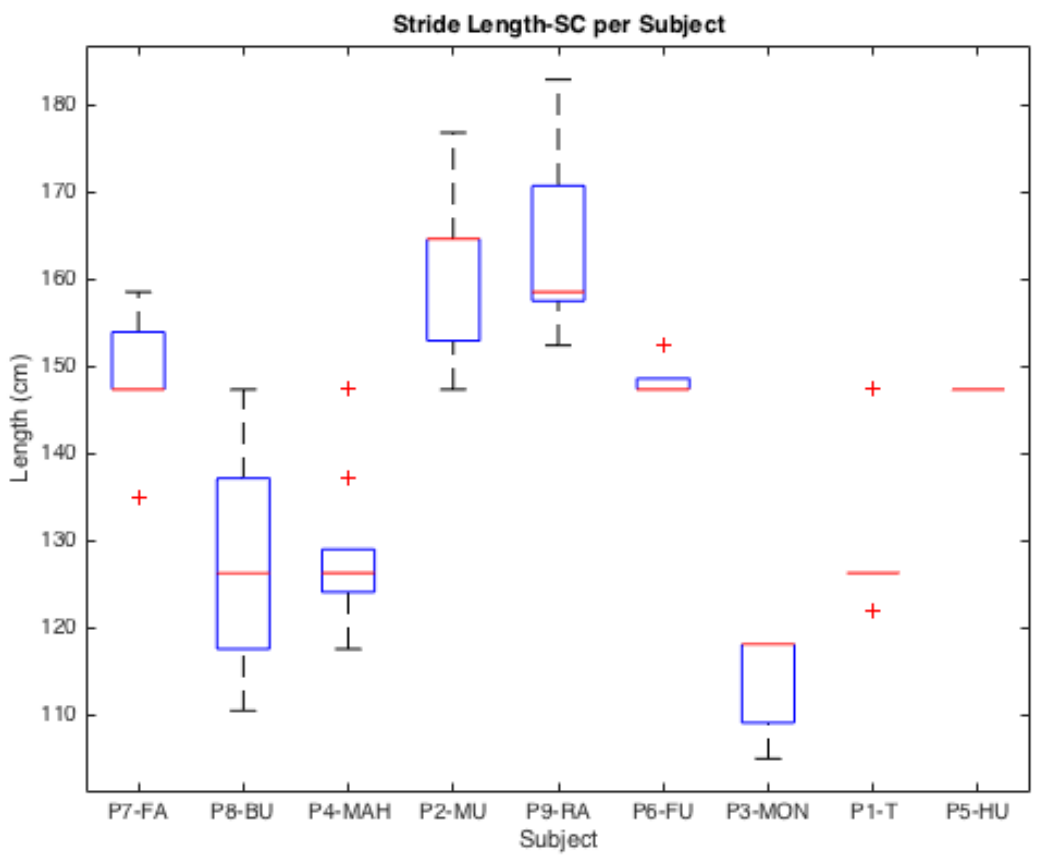

(d) 


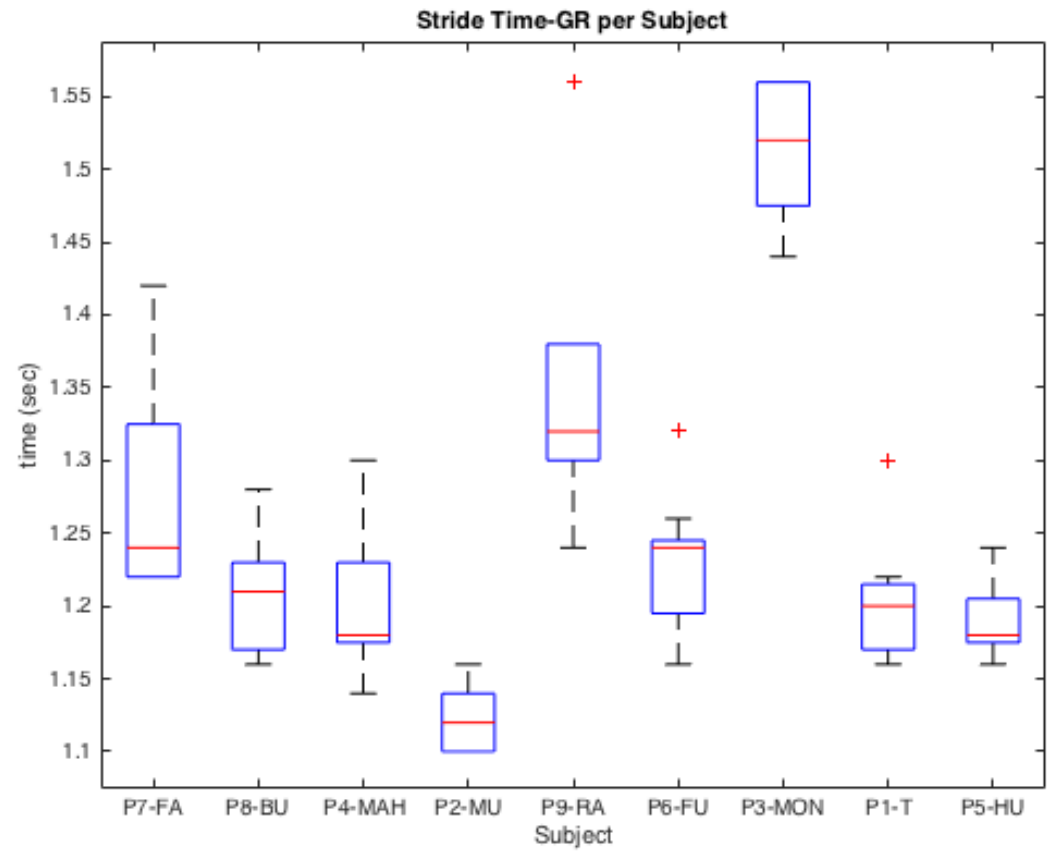

(e)

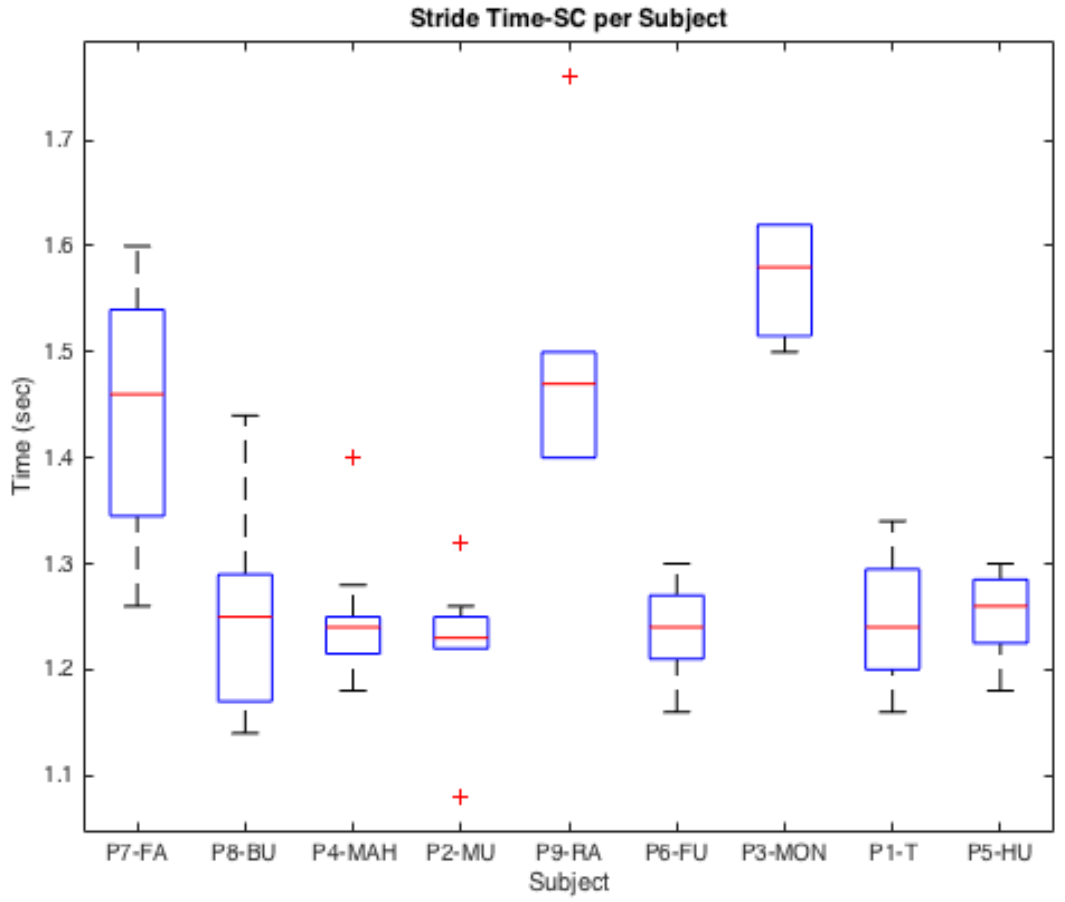

Figure 3-5 The mean, range, and outliers for walking speed, stride length, and stride time for both Smart carpet and GAITRite mat systems (a,b,c,d,e) 


\subsection{Conclusion}

We extracted the gait parameters from the smart carpet, and compared the results to the GAITRite mat, which was used as gold standard. Our algorithms achieved excellent agreement for walking speed, stride length and stride time between the two systems. The mean percentage error difference for walking speed is $1.43 \%$, stride length is $-4.32 \%$ and stride time is $-5.73 \%$. For walking speed, the standard deviation is 4.39 , which emphasizes that $68 \%$ of the error is within a $5 \%$. Correlations were calculated between GAITRite and smart carpet data for three gait parameters; walking speed, stride time and stride length. The $r$-squared of $93 \%$ for walking speed showed great agreement between the two systems. Similarly, we had agreement in stride length $(80 \%)$, and stride time (63\%). To get some improvements in the accuracy of measurements, we need to build higher resolution carpet, and enhance our algorithms to detect the footfalls. 


\section{CHAPTER 4: WAVEFORM STUDIES}

\subsection{Introduction}

Our system, the smart carpet, is designed to scavenge the signal from the environment. Signal scavenging originated from the concept of energy harvesting from the existing energy in the environment. The sensors made from a conductive material picks up stray $60 \mathrm{~Hz}$ noise [2]. When a subject steps or walks on the sensor, a change in the total charge of the conducting sensor sheet occurs causing the generated voltage level to increase or decrease depending on the step in or out of the sensor. The shape of the voltage waveform due to the amount of charge accumulated or discharged during this process is of interest. In previous work; fall detection [3] and gait estimation [5], we were interested on whether the sensor is active or not. However, we believe there may be more information in the scavenged signal. We investigated some of the characteristics of the sensor's accumulated charge or voltage level with the hope to answer the question: What other information can we get from the walk?

We studied the characteristics and the behavior of the sensor's scavenged signal. We constructed a single large sensor, onto which subjects performed multiple walks, and their data recorded and studied. The sensors' voltage waveforms behaved differently corresponding to different people, the material covering the sensors, and the environmental conditions. We performed a set of experiments on different configurations, number and characteristics of participants. To reduce the high variability we found it best to consider two sensors layouts: an18 x18 inch segment where four participants walked on the segment using only one step (right foot), and an 18 inch x 96 inch segment. 


\subsection{Waveform Characteristics of Scavenged Charge}

\subsubsection{Preliminary work}

We built a new sensor segment 8 feet long and 18 inches wide covered by vinyl, cloth carpet, or tiles as shown in Figure 4.1. This sensor segment allowed a participant to perform three steps per walk. This sensor layout allows us to analyze more temporal information rather than a single pulse of the generated voltage signal. Four different people walked on the sensor segment four times, each time maintaining the same walking speed and pace. Participants performed the walks within a single time period to reduce the effect of the weather such as temperature and relative humidity. These environmental conditions have an effect on static charge build. We used MSO4034 Tektronix Oscilloscope to record the generated digital signal for further analysis. We applied proper signal processing techniques; computing the signal's spectral density (Fourier Transform), total power, pulse width, and shape. Covering the sensor with the carpet produced cleaner and less noisy signal compared to vinyl cover. We
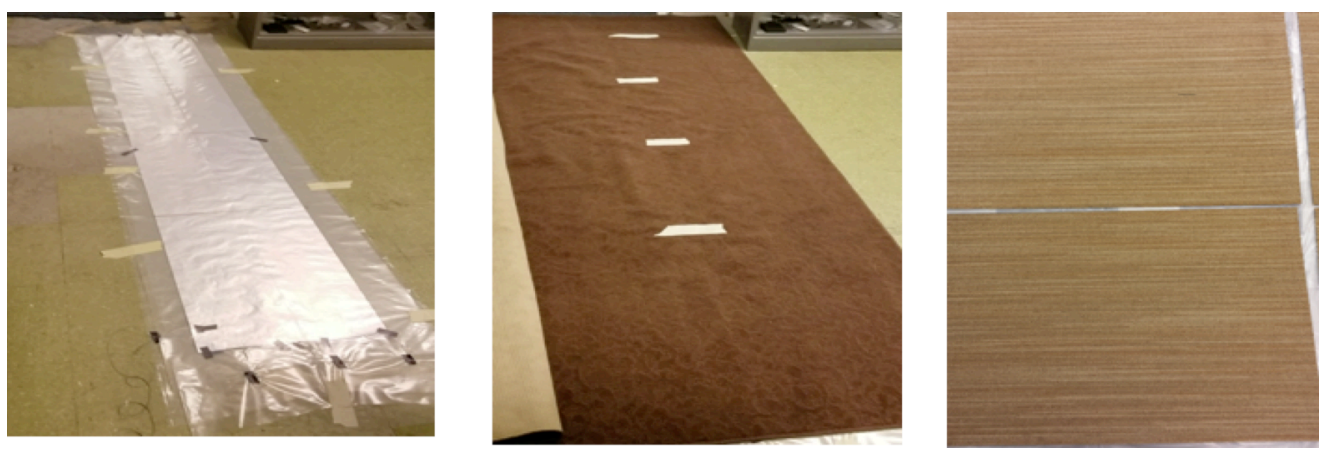

Figure 4-1 an 8-foot by 18-inch sensor segment covered by vinyl (left), cloth (middle), and tiles (right) 
repeated the same experiments for tiled carpet covering the sensors, which were later used for experiments as results showed more stable scavenged signals.

We designed a smaller $18 \times 18$ inches sensor segment. This layout goes naturally with the existing smart carpet system [3]. It is a little bigger than the existing $6 x 6$ inches sensors segments as we tried to get the foot to fit in one segment. Figure 4.2 shows the new sensor layout (left), the step of a participant fits in the sensor's area, and the generated voltage signal(right).
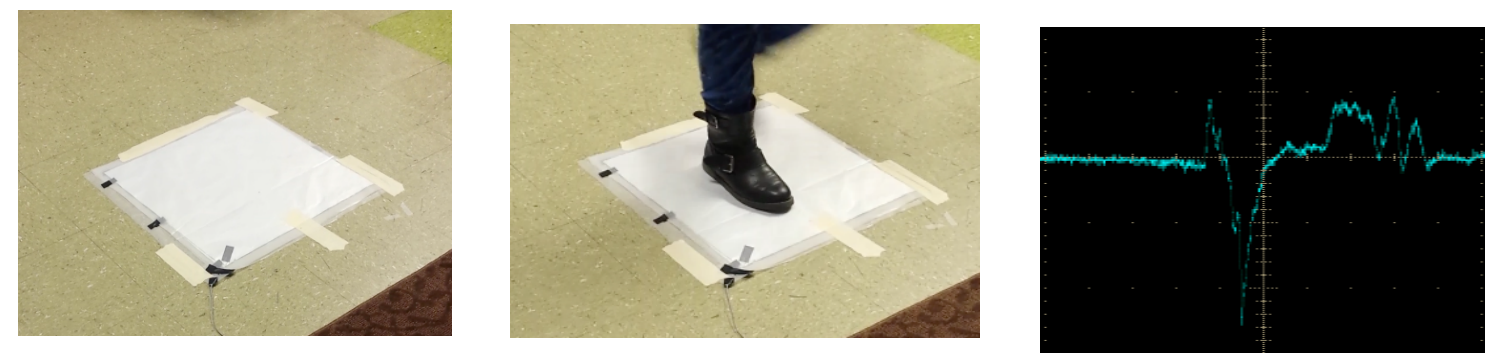

Figure 4-2. 18"x18" segment (left), one participant stepping on it (middle), and the generated signal (right) Our purpose was to see if this voltage had characteristics related to the walker's characteristics such as the weight or height. Clearly, the number of major peaks is related to the number of the steps. However, our first analysis showed that we couldn't relate the characteristics of the person depending on the analog signal generated such as signal power, the area under the curve, the signal width, or height of peaks. We did do better with computational intelligence techniques.

\subsubsection{Feature extraction}

As described in the previous section, we could not use the analog features like power, pulse width, number of peaks, area under the curve, etc. We decided to further investigate the non-analog features of the signals using Mel Frequency Cepstral 
Coefficient (MFCC) [30], which is a common and efficient technique for signal processing. This technique is well known in speech recognition [30,31]. We utilized this technique by extracting the first 13 MFCC features from the voltage signal, constructed training and testing data sets, and performed classification using $K$-Nearest Neighbor with $K$ was set to 1 .

\subsubsection{Experimental setup}

In this experiment we used the 8 -foot sensor segment covered by tiles. Seven adult participants performed 10 walks each in two scenarios; barefooted, and with shoes. We did this to eliminate the effect of the shoes material on the scavenged signal charge builds up. Table 4.1 shows the demographics of the participants.

TABle 4-1 SubJeCt’s Age, weight, HeIGHt, Shoe Size AND tyPe

\begin{tabular}{|l|l|l|l|l|l|}
\hline Subject & Age & Weight (lb.) & Height (inch) & Shoe size (inch) & Shoe type* \\
\hline P1 & 48 & 350 & 66 & 11 & Dress shoes/leather \\
\hline P2 & 33 & 300 & 69 & 10.5 & Leather \\
\hline P3 & 35 & 275 & 71 & 10 & Leather \\
\hline P4 & 35 & 215 & 69 & 9.5 & Tennis shoes leather \\
\hline P5 & 39 & 195 & 69 & 9.5 & Faux-leather heel \\
\hline P6 & 24 & 165 & 71 & 11 & Tennis shoes leather \\
\hline P7 & 55 & 140 & 68.5 & 9.5 & Sandals/leather \\
\hline *Experiments repeated one week later barefooted & & & \\
\hline
\end{tabular}

Same participants were asked to perform the same experiments barefooted, with pants raised near the knee to reduce the external effects on charge build up. Figure 4.3 shows 3 sample waveforms for participant number five (P5) wearing shoes and 
barefooted. We performed the same analog analysis we did previously for the four participants. We could not find discriminatory features. So, we shifted our gears towards extracting the MFCC features using formal computational intelligence techniques.
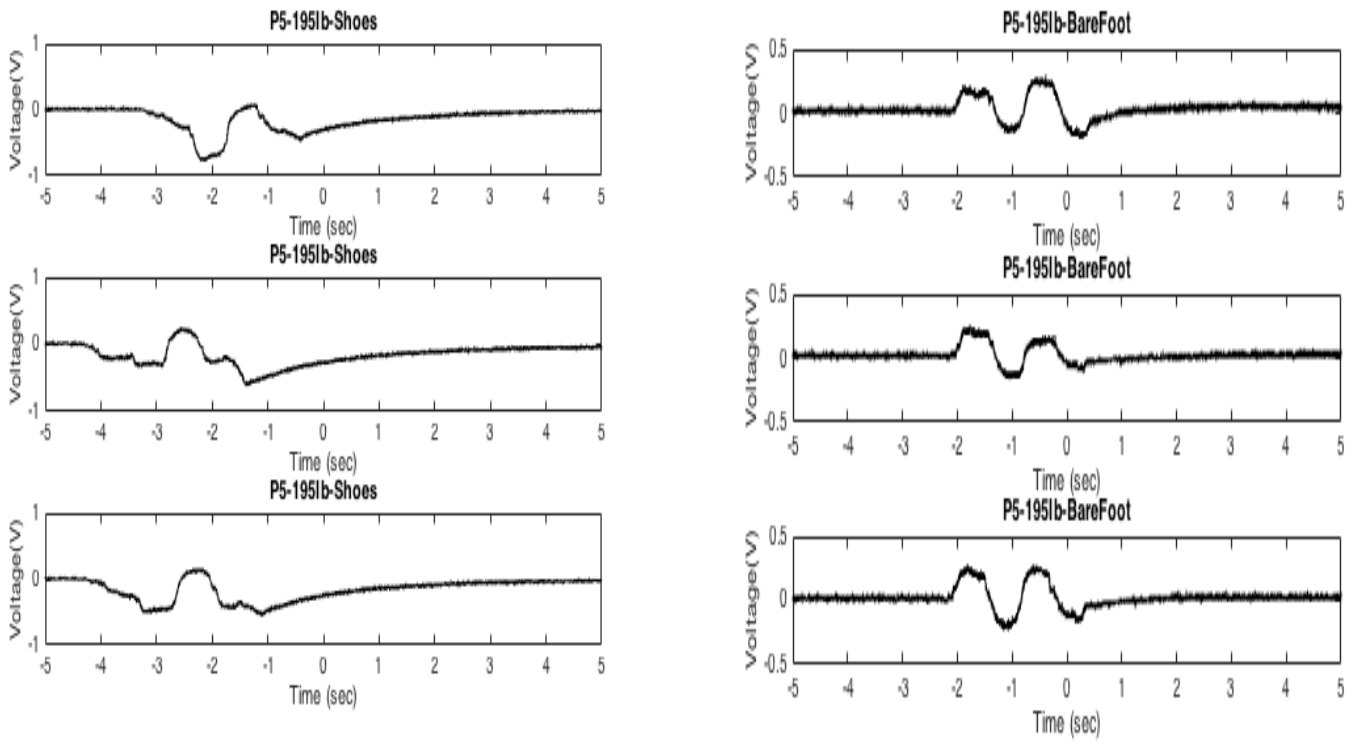

Figure 4-3 Sample waveforms generated for P5 walking 3 times with Shoes (Left), and barefoot (right)

\subsubsection{MFCC Features Extraction}

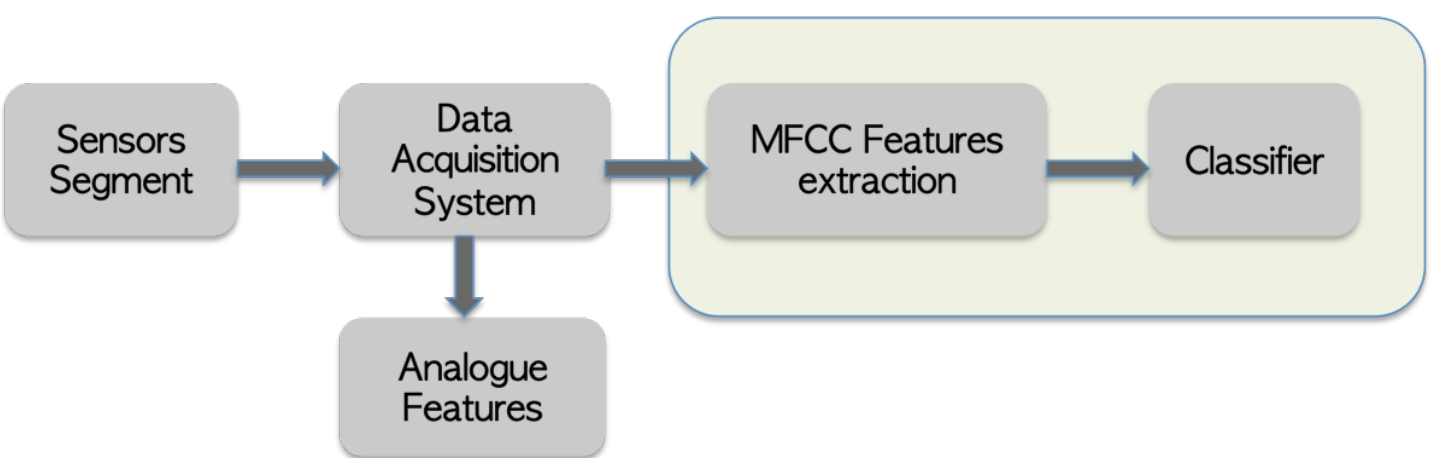

Figure 4-4 Step by step processing of the scavenged signal classification 
Figure 4.4 shows the top-level of the MFCC feature extraction process. A person walks on the sensor segment, and the scavenged signal is amplified and then measured by the oscilloscope that provides a file with digitized voltage values and time. The signal is analyzed using MFCC technique described in Figure 4.5. The output after applying MFCC is a matrix having features vectors extracted from the generated signal for one complete walk. In this matrix the rows represent the corresponding frame numbers and columns represent corresponding feature vector coefficients. The generated dataset is fed into Nearest Neighbor (NN) classifier, which classifies the walks performed by a participant using the distance between the MFCC feature matrices.

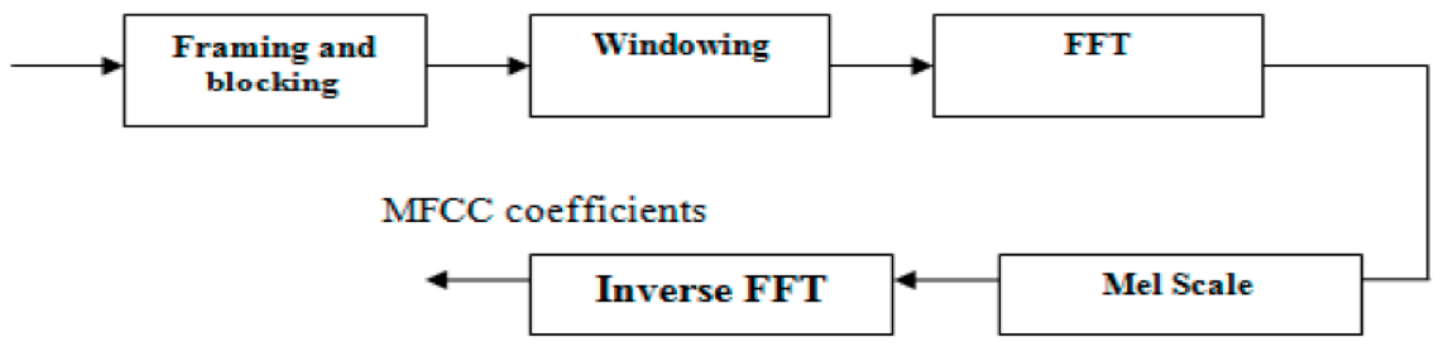

Figure 4-5 MFCC feature extraction process

Figure 4.5 shows the MFCC features extraction process, which can be described as follows:

\section{A. Framing and blocking}

The input signal size is 10,000 samples (sampling frequency $=1000$ samples/second). We segmented the input signal into a number of frames of size 256 samples with overlap of 100 samples. The rolling window feature allows reliable information compared to fixed windows sizes. The number of frames in each walk sample is 98 . 


\section{B. Windowing}

We used hamming window filter [34] represented by equation (1) of size $\mathrm{N}$, where $\mathrm{N}=256$ to smooth and minimize disruption at the start and end of the frames.

$$
w(n)=0.54-0.46 \cos \left(2 \pi \frac{n}{N}\right), \quad 0 \leq n \leq N
$$

\section{FFT (Fast Fourier Transform)}

Converts the time-domain points into the frequency domain to obtain the frequency spectral features of the signal. It is applied to each frame separately.

\section{Mel Scale (Mapping and Filtering)}

The FFT spectrum is mapped to Mel-Scale using equation (2). Frequency $\boldsymbol{f}$ ranges from 0 to $1000 \mathrm{~Hz}$. A triangular overlapping windows (filter banks), which is a set of triangular band pass filters (BPF), are equally spaced according to the Mel frequency. Those filters are multiplied by the FFT spectrum to estimate the energies at each frequency. The log of this energy is the Mel Spectrum, which is used to calculate the 13 coefficients of the Discrete Cosine Transform (DCT). [35]

$$
m_{f}=2595 \log _{10}\left(1+\frac{f}{700}\right)=1127 \ln \left(1+\frac{f}{700}\right)
$$

\section{E. DCT (Discrete Cosine Transform)}


DCT converts the log of Mel Spectrum into time domain. The output of the DCT is the MFCC vector for one frame. We need to repeat steps (D \& E) 98 times to generate the MFCC matrix for all the frames.

\subsubsection{Experimental results and Discussion}

\subsubsection{Analogue features}

The current system easily performs analog measurements, using the standard electronics. So we are very interested in analog voltage peaks, power, pule width, etc. Subjects performed the walks on different sensors surfaces; vinyl, threaded/cloth carpet or tiles. Results showed sensors covered with different material have correspondingly different signal behavior. The measured power (amount of charge accumulated) differs with different material used. Table 4-2 shows the average power measured for all four subjects' trials, performed separately, on vinyl, threaded/cloth carpet, and tiles. Clearly, we could not consistently relate the generated signal power to the weight or height of the

TABLE 4-2 AVERAGE POWER MEASURED USING VINYL, AND CARPET SURFACES

\begin{tabular}{|l|c|c|c|}
\hline Subject & $\begin{array}{c}\text { Vinyl } \\
\text { (Power Unit x1 e6) }\end{array}$ & $\begin{array}{c}\text { Threaded Carpet } \\
\text { (Power Unit x1e6) }\end{array}$ & $\begin{array}{c}\text { Tiles } \\
\text { (Power Unit x1e6) }\end{array}$ \\
\hline $\begin{array}{l}\text { Adult-male } \\
(200 \mathrm{lb} \text {. })\end{array}$ & 25.43 & 16.70 & 8.23 \\
\hline $\begin{array}{l}\text { Adult-female } \\
(150 \mathrm{lb} \text {. })\end{array}$ & 9.51 & 7.04 & 10.67 \\
\hline $\begin{array}{l}\text { Child-female } \\
(100 \mathrm{lb} \text {. })\end{array}$ & 9.29 & 3.33 & 2.40 \\
\hline $\begin{array}{l}\text { Child-male } \\
(50 \mathrm{lb} .)\end{array}$ & 4.11 & 1.73 & 6.0 \\
\hline
\end{tabular}

participants. Figure 4-6 shows samples of the scavenged signals from the 8-foot sensor 
segment covered by vinyl (top), tiles (middle), or threaded carpet (bottom) for a walk performed by a $200 \mathrm{lb}$. adult male. Four subjects repeated the same walks, and each subject generated different waveforms. We have traced the footfalls and observed that as the subjects took steps there was charge build up or discharge.
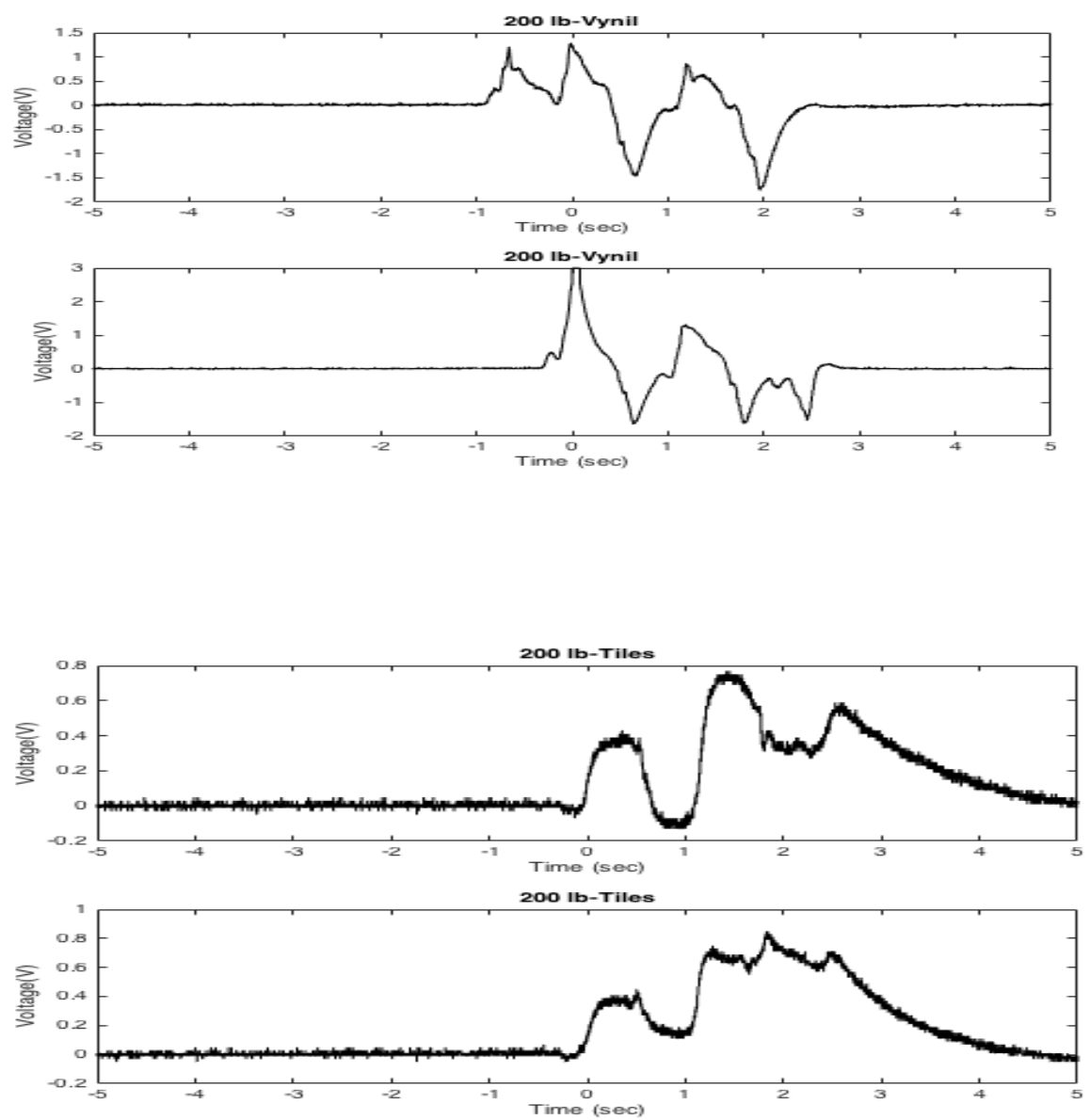

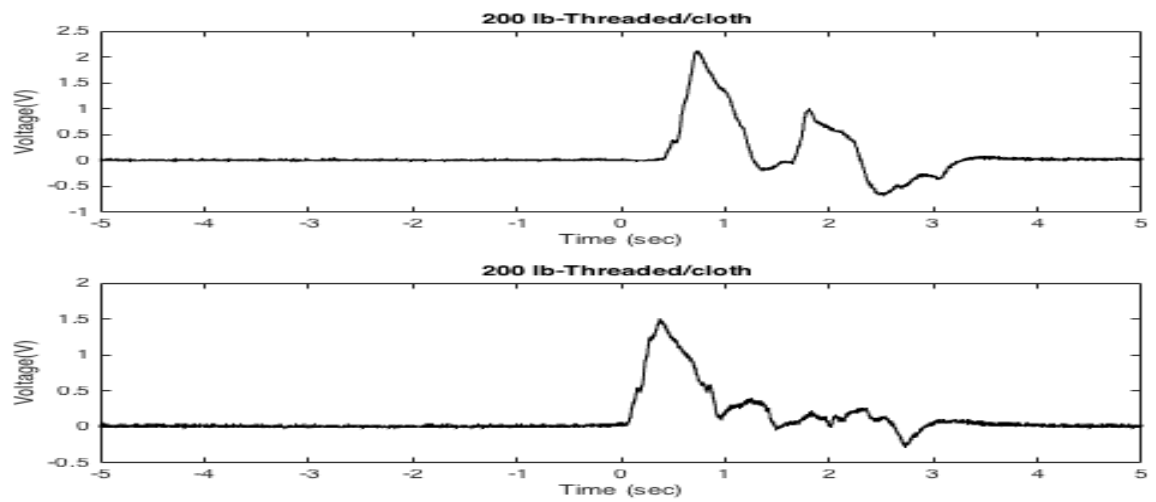

Figure 4-6 Samples of scavenged signal for a 200lb male walking on 8-Foot sensors segment covered by: vinyl (top), threaded/cloth (middle), and tiles (bottom)

However, we need to develop criteria and measurements to understand and interpret the scavenged signal, since different people have different waveforms, and charge build-up characteristics. It seems interesting to investigate considering other factors like weather, shoe type, sensor material, etc. We performed more walks under fixed weather conditions to study the effect of the shoe type, wearing only socks, and barefooted. As expected results showed that the abovementioned factors affect the charge build: Figure 4.7 shows the total power computed for the walks performed by a $200 \mathrm{lb}$. participant wearing shoes, socks, and barefooted. 


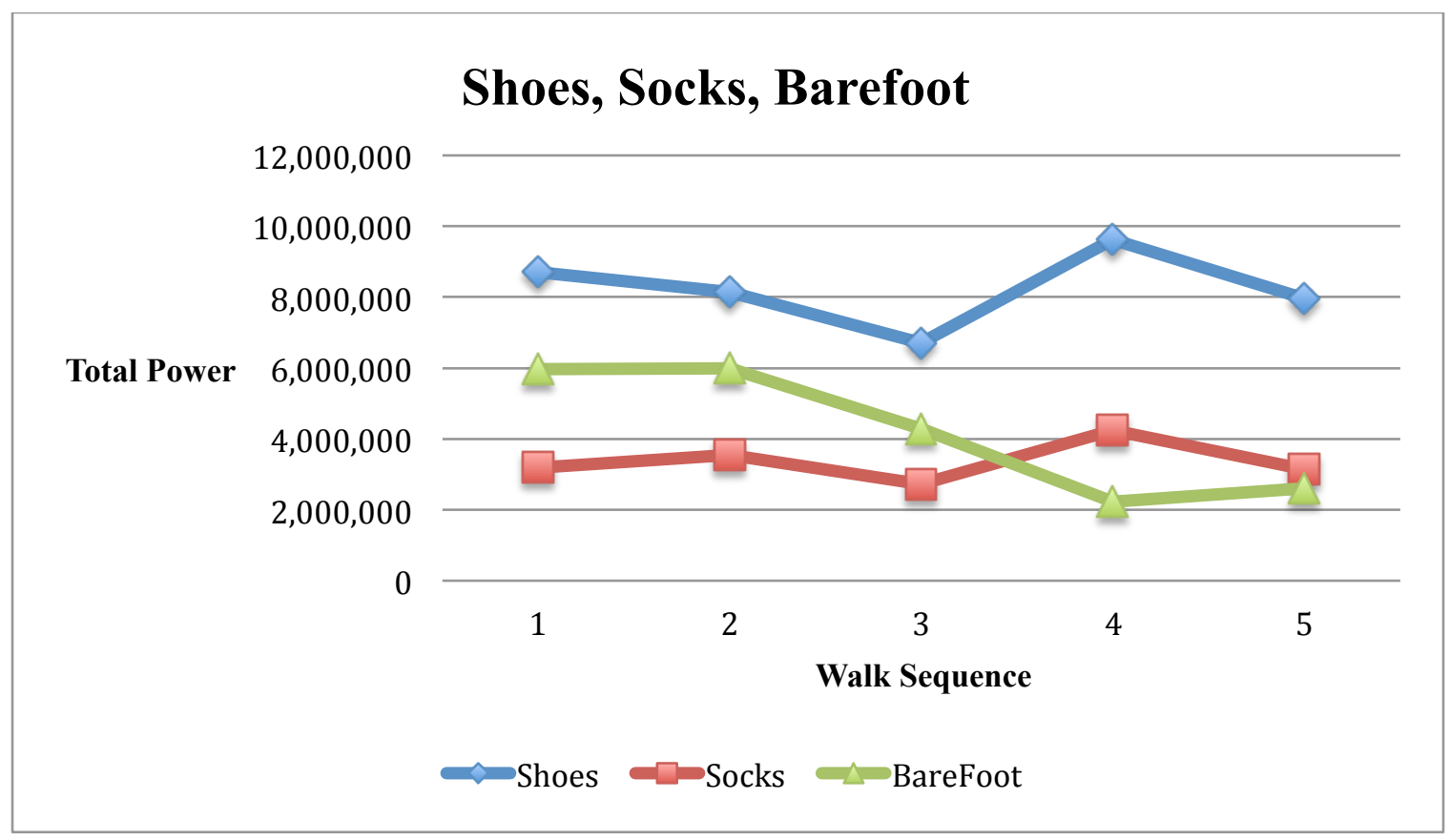

Figure 4-7 The total power computed for the walks performed by a $200 \mathrm{lb}$ subject wearing shoes, socks, and barefooted.

The Experiments performed by the seven volunteers did not change our conclusion or results that the analogue features alone are not enough to characterize a person, or to identify people based upon their scavenged signal.

\subsubsection{MFCC features}

Seven subjects performed 10 walking sequences on the 8 -foot sensor segment covered by tiles. They performed separate walks wearing shoes, and barefooted. The MFCC features matrix (13 (features) x 98 (frames)) is generated for each frame for the 98 frames forming a walking sequence. A total of 70 instances (featured matrices) were computed and aggregated into a dataset used for classification. We generated the dataset in a Weka [36] framework compatible format (.ARFF). Weka frameworks consist of a 
collection of classifiers and clusters algorithm that allowed us to use different classifiers. We used the Nearest Neighbor (1-NN) classifier. We divided the data into testing $(40 \%)$, and training $(60 \%)$. However, Weka allowed us to randomly divide the dataset into testing and training based on a variable percentage. We used three different training and testing schemes with the NN classifier: separate training and testing vectors, 10-fold cross validation, and percentage split. We present our results in a confusion matrix, where the diagonal entries represent the accuracy for the perspective class. Off diagonals are the misclassifications. Table 4.3 shows the confusion matrix for the seven persons walked on the carpet "Wearing Shoes" with $60 \%$ training and $40 \%$ testing scheme. The number of evaluation instances is 28 . The correctly classified instances are $23 / 28$ with accuracy of $82.14 \%$. The incorrectly classified instances are $5 / 28$ with misclassification rate $17.86 \%$. Table 4.4 shows the confusion matrix for the walks performed by the same seven subjects "Barefooted". The correctly classified instances are 19/28 with accuracy of $67.86 \%$. And misclassified instances are 9/28 with $32.14 \%$ misclassification rate. Table 4.5 and Table 4.6 show classifications using 10-fold cross validation scheme for "Wearing Shoes", and "Barefooted" respectively. 
TABLE 4-3 Classification RESUlTS FOR "SubJECTS WEARING SHOES" TRAINING (60\%) AND teST $(40 \%)$ DATA

\begin{tabular}{|l|l|l|l|l|l|l|l|l|}
\hline & P1 & P2 & P3 & P4 & P5 & P6 & P7 & Accuracy \\
\hline P1 & $\mathbf{2}$ & 1 & 0 & 0 & 1 & 0 & 0 & $50 \%$ \\
\hline P2 & 0 & $\mathbf{4}$ & 0 & 0 & 0 & 0 & 0 & $100 \%$ \\
\hline P3 & 0 & 0 & 4 & 0 & 0 & 0 & 0 & $100 \%$ \\
\hline P4 & 0 & 0 & 0 & $\mathbf{4}$ & 0 & 0 & 0 & $100 \%$ \\
\hline P5 & 0 & 0 & 0 & 1 & $\mathbf{3}$ & 0 & 0 & $75 \%$ \\
\hline P6 & 0 & 0 & 0 & 0 & 0 & $\mathbf{3}$ & 1 & $75 \%$ \\
\hline P7 & 0 & 0 & 0 & 0 & 1 & 0 & $\mathbf{3}$ & $75 \%$ \\
\hline \multicolumn{2}{|l|}{ Overall Accuracy } & $\mathbf{8 2 . 1 4} \%$ & \multicolumn{7}{|c|}{ Overall ROC Area } & $\mathbf{0 . 8 8 9}$ & \\
\hline
\end{tabular}

TABle 4-4 Classification RESUlTS FOR “SUbJECTS BAREFOOTED” TRAining (60\%) AND TEST (40\%) DATA

\begin{tabular}{|l|l|l|l|l|l|l|l|l|}
\hline & P1 & P2 & P3 & P4 & P5 & P6 & P7 & Accuracy \\
\hline P1 & $\mathbf{2}$ & 0 & 0 & 1 & 0 & 1 & 0 & $50 \%$ \\
\hline P2 & 0 & $\mathbf{4}$ & 0 & 0 & 0 & 0 & 0 & $100 \%$ \\
\hline P3 & 0 & 0 & $\mathbf{1}$ & 1 & 2 & 0 & 0 & $25 \%$ \\
\hline P4 & 0 & 0 & 1 & $\mathbf{3}$ & 0 & 0 & 0 & $75 \%$ \\
\hline P5 & 0 & 0 & 0 & 0 & $\mathbf{3}$ & 1 & 0 & $75 \%$ \\
\hline P6 & 0 & 0 & 0 & 0 & 0 & $\mathbf{4}$ & 0 & $100 \%$ \\
\hline P7 & 1 & 0 & 0 & 1 & 0 & 0 & $\mathbf{2}$ & $50 \%$ \\
\hline Overall Accuracy & \multicolumn{2}{|c|}{$\mathbf{6 7 . 8 6} \%$} & \multicolumn{7}{|c|}{ Overall ROC Area } & $\mathbf{0 . 8 1 3}$ & \\
\hline
\end{tabular}


TABLE 4-5 CLASSIFICATION RESULTS FOR “WEARING SHOES" 10-FOLD CROSS VALIDATION

\begin{tabular}{|l|l|l|l|l|l|l|l|l|}
\hline & P1 & P2 & P3 & P4 & P5 & P6 & P7 & Accuracy \\
\hline P1 & $\mathbf{4}$ & $\mathbf{2}$ & $\mathbf{0}$ & $\mathbf{0}$ & $\mathbf{3}$ & $\mathbf{1}$ & $\mathbf{0}$ & $40 \%$ \\
\hline P2 & $\mathbf{0}$ & $\mathbf{8}$ & $\mathbf{0}$ & $\mathbf{0}$ & $\mathbf{0}$ & $\mathbf{2}$ & $\mathbf{0}$ & $80 \%$ \\
\hline P3 & $\mathbf{0}$ & $\mathbf{0}$ & $\mathbf{1 0}$ & $\mathbf{0}$ & $\mathbf{0}$ & $\mathbf{0}$ & $\mathbf{0}$ & $100 \%$ \\
\hline P4 & $\mathbf{0}$ & $\mathbf{0}$ & $\mathbf{0}$ & $\mathbf{1 0}$ & $\mathbf{0}$ & $\mathbf{0}$ & $\mathbf{0}$ & $100 \%$ \\
\hline P5 & $\mathbf{1}$ & $\mathbf{0}$ & $\mathbf{0}$ & $\mathbf{1}$ & $\mathbf{8}$ & $\mathbf{0}$ & $\mathbf{0}$ & $80 \%$ \\
\hline P6 & $\mathbf{0}$ & $\mathbf{0}$ & $\mathbf{0}$ & $\mathbf{0}$ & $\mathbf{0}$ & $\mathbf{7}$ & $\mathbf{3}$ & $70 \%$ \\
\hline P7 & $\mathbf{0}$ & $\mathbf{0}$ & $\mathbf{0}$ & $\mathbf{0}$ & $\mathbf{0}$ & $\mathbf{6}$ & $\mathbf{4}$ & $40 \%$ \\
\hline \multicolumn{2}{|l|}{ Overall Accuracy } & $\mathbf{7 2 . 8 6 \%}$ & \multicolumn{2}{|c|}{ Overall ROC Area } & $\mathbf{0 . 8 4 2}$ & \\
\hline
\end{tabular}

TABLE 4-6 CLASSIFICATION RESULTS FOR “BAREFOOTED” 10-FOLD CROSS VALIDATION

\begin{tabular}{|l|l|l|l|l|l|l|l|l|}
\hline & P1 & P2 & P3 & P4 & P5 & P6 & P7 & Accuracy \\
\hline P1 & $\mathbf{7}$ & $\mathbf{1}$ & $\mathbf{0}$ & $\mathbf{1}$ & $\mathbf{0}$ & $\mathbf{1}$ & $\mathbf{0}$ & $70 \%$ \\
\hline P2 & $\mathbf{0}$ & $\mathbf{6}$ & $\mathbf{0}$ & $\mathbf{4}$ & $\mathbf{0}$ & $\mathbf{0}$ & $\mathbf{0}$ & $60 \%$ \\
\hline P3 & $\mathbf{0}$ & $\mathbf{0}$ & $\mathbf{5}$ & $\mathbf{4}$ & $\mathbf{1}$ & $\mathbf{0}$ & $\mathbf{0}$ & $50 \%$ \\
\hline P4 & $\mathbf{0}$ & $\mathbf{0}$ & $\mathbf{2}$ & $\mathbf{8}$ & $\mathbf{0}$ & $\mathbf{0}$ & $\mathbf{0}$ & $80 \%$ \\
\hline P5 & $\mathbf{0}$ & $\mathbf{0}$ & $\mathbf{0}$ & $\mathbf{0}$ & $\mathbf{8}$ & $\mathbf{2}$ & $\mathbf{0}$ & $80 \%$ \\
\hline P6 & $\mathbf{1}$ & $\mathbf{0}$ & $\mathbf{1}$ & $\mathbf{1}$ & $\mathbf{0}$ & $\mathbf{7}$ & $\mathbf{0}$ & $70 \%$ \\
\hline P7 & $\mathbf{1}$ & $\mathbf{0}$ & $\mathbf{0}$ & $\mathbf{0}$ & $\mathbf{0}$ & $\mathbf{0}$ & $\mathbf{9}$ & $90 \%$ \\
\hline Overall Accuracy & \multicolumn{7}{|c|}{$\mathbf{7 1 . 4 3 \%}$} & \multicolumn{7}{|c|}{ Overall ROC Area } & $\mathbf{0 . 8 3 3}$ & \\
\hline
\end{tabular}


We used 'Wear Shoes' data as training set, and then evaluated the classifier with the "Barefooted" data. The result in Table 4.7 shows $18.57 \%$ classification accuracy, and $81.43 \%$ misclassification rate.

TABle 4-7 Classification RESULtS FOR TRAINING WITH “WEAR SHOES” AND TESTING BY "BAREFOOTED"

\begin{tabular}{|l|l|l|l|l|l|l|l|l|}
\hline & P1 & P2 & P3 & P4 & P5 & P6 & P7 & Accuracy \\
\hline P1 & $\mathbf{2}$ & $\mathbf{2}$ & $\mathbf{0}$ & $\mathbf{0}$ & $\mathbf{0}$ & $\mathbf{6}$ & $\mathbf{0}$ & $20 \%$ \\
\hline P2 & $\mathbf{0}$ & $\mathbf{5}$ & $\mathbf{0}$ & $\mathbf{0}$ & $\mathbf{0}$ & $\mathbf{3}$ & $\mathbf{2}$ & $50 \%$ \\
\hline P3 & $\mathbf{2}$ & $\mathbf{6}$ & $\mathbf{0}$ & $\mathbf{0}$ & $\mathbf{0}$ & $\mathbf{3}$ & $\mathbf{2}$ & $0 \%$ \\
\hline P4 & $\mathbf{3}$ & $\mathbf{4}$ & $\mathbf{0}$ & $\mathbf{0}$ & $\mathbf{0}$ & $\mathbf{3}$ & $\mathbf{0}$ & $0 \%$ \\
\hline P5 & $\mathbf{1 0}$ & $\mathbf{0}$ & $\mathbf{0}$ & $\mathbf{0}$ & $\mathbf{0}$ & $\mathbf{0}$ & $\mathbf{0}$ & $0 \%$ \\
\hline P6 & $\mathbf{8}$ & $\mathbf{1}$ & $\mathbf{0}$ & $\mathbf{0}$ & $\mathbf{0}$ & $\mathbf{1}$ & $\mathbf{0}$ & $10 \%$ \\
\hline P7 & $\mathbf{2}$ & $\mathbf{1}$ & $\mathbf{0}$ & $\mathbf{0}$ & $\mathbf{0}$ & $\mathbf{2}$ & $\mathbf{5}$ & $50 \%$ \\
\hline Overall Accuracy & $\mathbf{1 8 . 5 7 \%}$ & \multicolumn{2}{|c|}{ Overall ROC Area } & $\mathbf{0 . 5 3 5 5}$ & \\
\hline
\end{tabular}

We then combined both data sets and used 10-fold cross validation scheme. Results in Table 4.8 shows a $66.43 \%$ accuracy rate with overall ROC area 0.804 . The participants performed the walks in two different dates approximately a week a part but within the same time period of the day.

Participant (P5) performed set of experiments in three different times of one day: morning, afternoon, and evening. Using the same classification settings, as before, results showed an overall accuracy of $86.67 \%$. All Afternoon instances were $100 \%$ correctly classified. 
TABLE 4-8 ClassificATION RESULTS FOR COMBINED DATA SET OF “WEAR SHoes” AND

“BAREFOOTED” USING 10-FOLD CROSS VALIDATION

\begin{tabular}{|c|c|c|c|c|c|c|c|c|}
\hline & P1 & P2 & P3 & P4 & P5 & P6 & P7 & Accuracy \\
\hline P1 & 7 & 3 & 1 & $\mathbf{0}$ & 5 & 3 & 1 & $35 \%$ \\
\hline P2 & 1 & 12 & 1 & 4 & $\mathbf{0}$ & 2 & $\mathbf{0}$ & $60 \%$ \\
\hline P3 & 1 & $\mathbf{0}$ & 15 & 4 & $\mathbf{0}$ & $\mathbf{0}$ & $\mathbf{0}$ & $75 \%$ \\
\hline P4 & $\mathbf{0}$ & 2 & 3 & 15 & $\mathbf{0}$ & $\mathbf{0}$ & $\mathbf{0}$ & $75 \%$ \\
\hline P5 & $\mathbf{0}$ & $\mathbf{0}$ & $\mathbf{0}$ & 1 & 17 & 2 & $\mathbf{0}$ & $85 \%$ \\
\hline P6 & 2 & $\mathbf{0}$ & 1 & 1 & $\mathbf{0}$ & 14 & 2 & $70 \%$ \\
\hline P7 & 1 & $\mathbf{0}$ & $\mathbf{0}$ & 1 & $\mathbf{0}$ & 5 & 13 & $65 \%$ \\
\hline \multicolumn{2}{|c|}{ Overall Accuracy } & \multicolumn{2}{|c|}{$66.43 \%$} & \multicolumn{3}{|c|}{ Overall ROC Area } & 0.804 & \\
\hline
\end{tabular}

\subsubsection{Walking speed}

We calculated the walking speed for the seven people who performed the experiments for the two scenarios "barefooted" and "wearing shoes". All experiments were performed on the sensors segment of 8 -foot size. Each participant walked 3 steps. We calculated the walking speed by dividing the distance travelled over the ambulation time. Table 4.9 shows the average walking speed for the seven participants for both "barefooted" and "wearing shoes". Figure 4.8 shows the average walking speed for the participants who performed the walks wearing shoes, and barefooted. The percentage relative difference is calculated with barefooted used as reference. The average was $6.06 \%$ but one outlier had a maximum percentage difference of $14.8 \%$. We did not 
instruct the subjects who performed the experiments to control or keep the same walking pace.

TABLE 4-9 AVERAGE WALKING SPEED FOR BOtH SCENARIOS: "WEAR SHOES", AND "BAREFOOTED"

\begin{tabular}{|c|c|c|c|}
\hline Subject & $\begin{array}{c}\text { Walking Speed } \\
(\mathbf{c m} / \mathbf{s e c}) \\
\text { Wear Shoes }\end{array}$ & $\begin{array}{c}\text { Walking Speed } \\
\text { (cm/sec) }\end{array}$ & $\begin{array}{c}\text { Percentage relative } \\
\text { Barefooted }\end{array}$ \\
\hline P1 & 72.36 & 75.72 & 4.4 \\
\hline P2 & 76.91 & 76.68 & 0.3 \\
\hline P3 & 84.96 & 92.71 & 8.4 \\
\hline P4 & 80.21 & 83.52 & 4 \\
\hline P5 & 84.07 & 98.73 & 4.8 \\
\hline P6 & 98.32 & 93.78 & 5.7 \\
\hline P7 & 86.46 & 82.81 & $6.06 \%$ \\
\hline \multicolumn{2}{|r|}{} & Average & \\
\hline
\end{tabular}

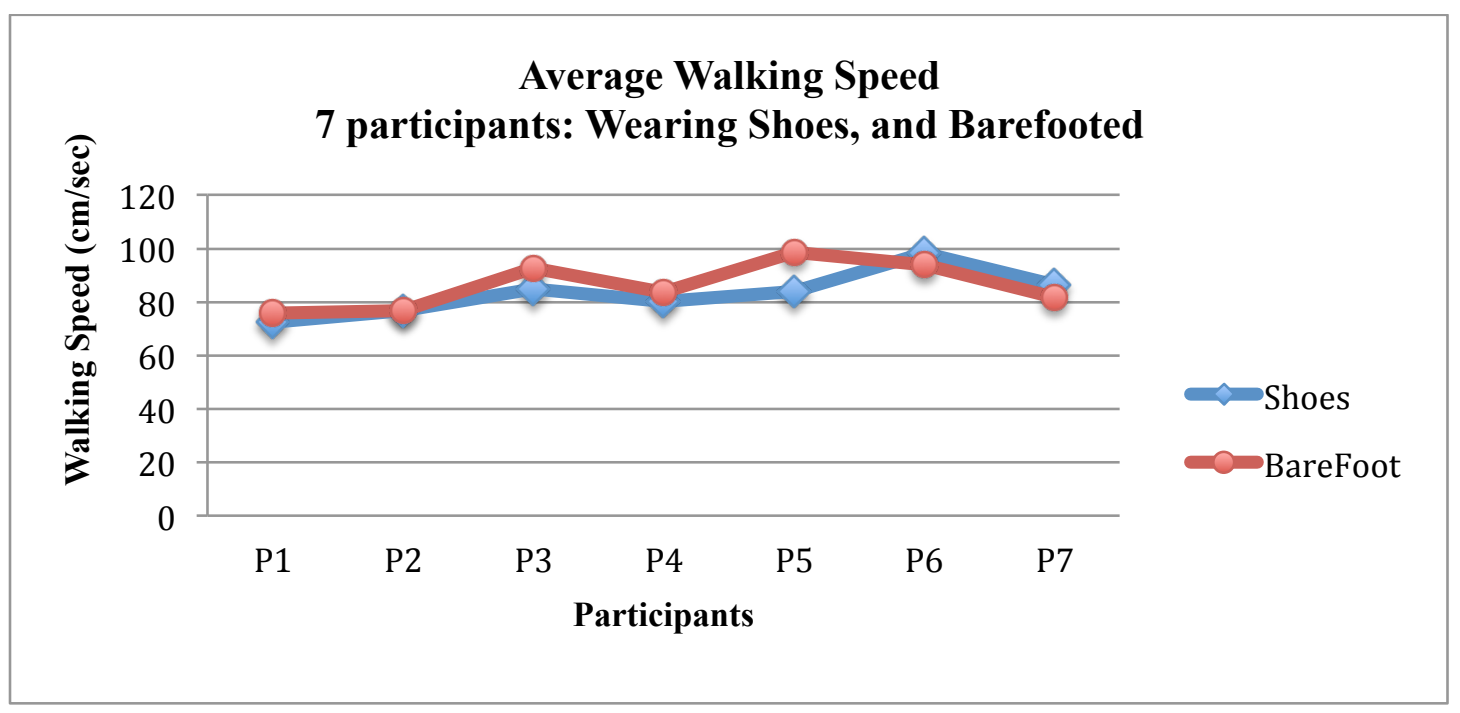

Figure 4-8 The average walking speed for the participant who performed the walks wearing shoes, and barefooted. The percentage relative difference is calculated with barefooted used as reference. 


\subsection{Conclusion}

We studied the characteristics and the behavior of the sensor's scavenged signal. We built a single large sensor, where subjects performed multiple walks on the sensor, and their data recorded and studied. The sensors' voltage waveforms behaved differently corresponding to different people and set of walking trials. By inspection, we could not distinguish different people due to the high variability like the covering material, shoe type, and the environmental conditions, which affect the behavior of the scavenged signal. However, we were able to use computational features, by extracting the Mel Frequency Cepstral Coefficients (MFCC), and using a formal computation intelligence to distinguish different people with an average accuracy of $82 \%$, given that the experiments were performed within the same day. 


\section{CHAPTER 5: MULTIPLE PEOPLE COUNT}

\subsection{Introduction}

With this material, we produced a paper titled "Counting Multiple People on a Floor Based Array sensor system". The paper was submitted to HIMS'17 - The 3rd International Conference on Health Informatics and Medical Systems [6]. We described the development and evaluation of algorithms to count the number of people walking on the smart carpet in controlled lab settings using the smart carpet. Data was collected from 4 participants who performed total of 10-walk sequences per walking scenario. Six testing scenarios developed; Individual, two people walking in the Same direction, two people walking in the Opposite direction, three people walking in the Same direction, four people walking in the Same direction, and four people walking in the Opposite direction. Our results showed that we could count and monitor individual and multiple people walking on the carpet with an accuracy of up to $100 \%$, when doing total counts. 


\title{
5.2 Manuscript: Counting Multiple People on a Floor Based Array sensor system
}

Fadi Muheidat and Harry W. Tyrer

Electrical and Computer Engineering department, University of Missouri, Columbia, USA

\begin{abstract}
We have developed a context-aware system that uses the functionality associated with the Internet of Things (IOT). We have a floor based array sensor system, which we call the Smart Carpet, which recognizes a person walking or falling, reports the fall and stores the data for regular evaluation of the gait parameters. These all have medical benefits. Here we report the improvement, which counts the number of individuals walking on the carpet. We used two methods to perform the count; the number of active sensors at given time, and the number of unique subgroups formed by the activated sensors using the connected component labeling algorithm for varying number of frames in the sliding window mainly 3, 5 and 9 frames. Our results showed that we could count and monitor individual and multiple people walking on the carpet with an average accuracy of $100 \%$. We use the carpet as a component of an automated health monitoring system, which helps enable independent living for elderly people and provide a practical smart home environment that improves quality of life, reduces healthcare costs and promotes independence.
\end{abstract}

Keywords: Sensing Floor, Patient Monitoring, Detecting People, Data Acquisition, Data Mining, Internet of Things (IOT), Smart home. 


\section{$5.2 \backslash 1$.Introduction}

The number of people aged 65 and older is growing worldwide. The US population will have one in five people 65 or older by 2030 [1]. The research community is innovating new technologies to help assistive living. However, the challenge is to have unobtrusive and user-friendly, unobserved, hand-free and affordable system that supports assisted living of elderly in their homes or in nursing houses.

Systems have been proposed and developed which can be categorized into wearable (accelerometer and gyroscope) [2], and non-wearable (context-aware) [3, 4, 5, 6, 7, 8]. These systems can be used to detect falls, estimate gait, monitor elderly activities. In addition, they can recognize, count people, and monitor their activities.

Wearables sensors systems are effective devices to detect and recognize the location and activity of people. However, they are obtrusive, must be worn at all times and need continuous power (batteries). In addition, it is not possible to anonymously count and detect people without previous knowledge of the person's wearable device.

Alternatively, context aware systems overcome some of these issues. In video monitoring systems, the vision techniques filter the images at the device level due to privacy concerns. However, the users still have the feeling that of being watched. Kinect, a videobased system uses skeleton tracking to detect people with good resolution has value [9, 10]. However, it suffers degraded performance with occlusion, and limited depth range. Multifunction radar systems [11] proved to be promising solution in detecting humans and their activities even behind walls or foliage, yet they suffer classifications accuracy for other barriers and movement gestures. Microphone array sensors [7, 12] suffer from noise and multiple interference. 
Our lab uses context-aware, non-computer-vision based human recognition and fall detection system. It is a floor based array sensors system, i.e. smart carpet [13], which is completely private. One installs it in the home or apartment and additionally has usefulness in places where traditional sensing system might suffer complications like occlusion. The smart carpet system includes the sensor data acquisition, data manipulating, data reading, storage, display, and communication. The system operates by detecting the person's movement and storing the floor sensor data. The motion on the carpet activates a set of sensors that outputs a voltage signal. The system amplifies the signal, digitizes it, and then translates it into a frame for further processing. We ran computational intelligence algorithms to measure and estimate people's gait, and detect falls. Our goal is to accurately recognize, count and monitor the movements of the individuals walking on the smart carpet system.

We organized this paper as follows. First, the methodology, which includes an overview of the system we developed and installed in lab settings. We describe algorithms used to count the number of people walking on the carpet. Third, we show experimental results for different walking scenarios performed by volunteers. Finally, we discuss the achieved results, limitations and future work. 


\section{2\2.Methodology}

\section{a. System overview}

The smart carpet system, as shown in Figure 1, consists of the Smart Carpet sensors laid under the mat, data acquisition system, and processor. The signal scavenging sensors connect to the data acquisition system that scans at configurable speeds depending on the size and number of sensors. Signals convert to digital values using 10bit Analog to Digital convertor, and microprocessor further thresholds providing a 1 for

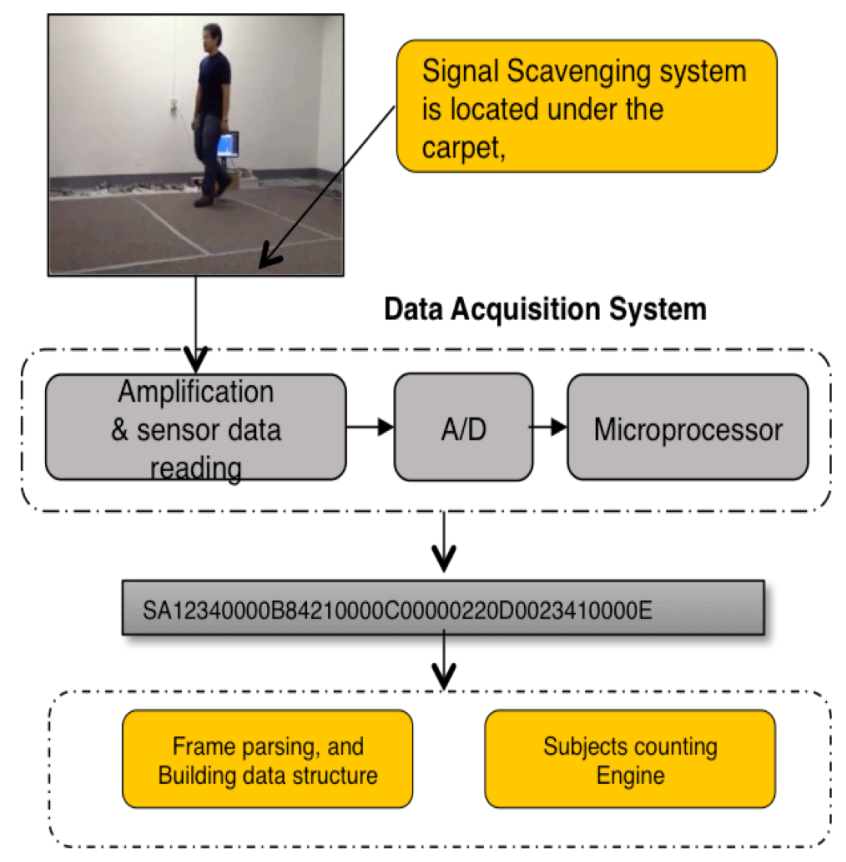

Figure 1 System overview

activated and 0 for not activated sensor, as well as formatting the frame with an $\mathrm{S}$ for start and $\mathrm{E}$ for end. A computer then reads this scan as an ASCII frame. The software components process the data frames, and use different computational intelligence methods to perform the required operations like fall detection, gait estimation, data 
visualization, and notification. Additionally, the system can show the signal data scavenged by the sensor for fine-tuning of the system parameters.

The system consists of sensor array made into four segments $\mathbf{A}, \mathbf{B}, \mathbf{C}$, and $\mathbf{D}$. Each segment is connected to data acquisition system. Each segment has 32 sensors. Segment D was turned off for the sake of this project. The layout of the carpet segments

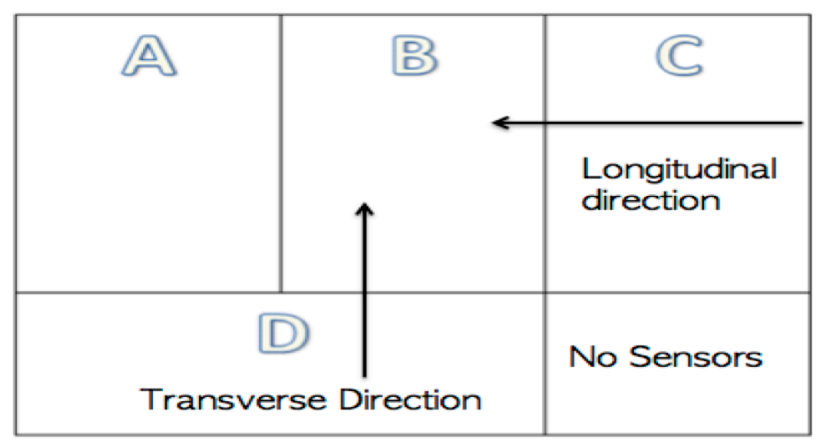

Figure 2. Carpet layout: Active segments A, B and C. Sensors in $\mathbf{D}$ are turned off

shown in Figure 2 Walking across the carpet from $\mathbf{A}$ to $\mathbf{C}$ or $\mathbf{C}$ to $\mathbf{A}$ (Longitudinal Direction) will require longer time, longer travelled distance, and more activated sensors count. Compared to waking from segment D direction bottom up (Transverse Direction). We made use of a binary display of the activated and non-activated sensors on the carpet to see the traversal of the individuals.

\section{b. Experiments and counting algorithms}

We collected data from four different people. As listed in Table I, each person, individually, performed 10 walk trials in traverse direction from bottom of segment $\mathbf{A}$ then to segment $\mathbf{B}$ and back to the beginning. Then, multiple persons participated in 2 people, 3 people and four people walk trials for 10 times each. The smart carpet data acquisition system scans the carpets at 9 frames per second. Each frame consists of 128 
sensors, where all segment D sensors turned off. However, we used them to build $12 \times 12$ binary image. Where ' 1 ' means, the sensor is activated, and ' 0 ' means it is not activated. This image becomes the base data structure to perform computation to recognize people

TABLE I SubJECT’S AgE, WeIGHT, AND HeIGHT

\begin{tabular}{|l|l|l|l|}
\hline \multicolumn{1}{|c|}{ Subject } & \multicolumn{1}{|c|}{ Weight } & \multicolumn{1}{|c|}{ Height } & Age \\
\hline Male - Adult & $200 \mathrm{lb} ., 90.72 \mathrm{Kg}$ & $5^{\prime} 9^{\prime \prime}, 174 \mathrm{~cm}$ & 40 \\
\hline Female- Adult & $150 \mathrm{lb}, 68 \mathrm{Kg}$ & $5^{\prime} 3^{\prime \prime}, 160 \mathrm{~cm}$ & 31 \\
\hline Female- Child & $98.4 \mathrm{lb} ., 44.63 \mathrm{Kg}$ & $4^{\prime} 8^{\prime \prime}, 142 \mathrm{~cm}$ & 12 \\
\hline Male - Child & $49.7 \mathrm{lb} ., 22.5 \mathrm{Kg}$ & $3^{\prime} 11^{\prime \prime}, 119 \mathrm{~cm}$ & 8 \\
\hline
\end{tabular}

on the carpet. We used Connected Component Labeling (CCL) algorithm as described in [14], we applied the same procedure for both single frames and window size of frames encompassing variable number of frames: 3, 5, and 9. Each window corresponds to time (WS $=3$ frames correspond to 0.2 seconds, WS $=5$ frames correspond to 0.5 seconds, and WS $=9$ frames corresponds to 1 seconds) $\boldsymbol{W S}=$ total number of frames corresponds to total travel / ambulation time). We used to 8-connect neighborhood [15] for our experiments to ensure we do not ignore the effect of interference among the sensors, and so we have biased results. We used a hybrid model of both the number of subgroups formed by the neighboring activated sensors, and the count of the subgroups formed by individual activated sensor that are not direct neighbors (outside the 8 neighborhood).

Another method we used is the count of the total sensors activated for full walk, and then divided by the average number of sensors activated per individual(s) who performed the walk. For example, the "Two people walking in opposite directions", we 
took the average count of active sensor performed by the two people when they walk alone on the carpet, and then divided the total active sensors of the full walk by this average number to count number of people.

\subsection{Experimental Results}

We performed 10 experiments for each scenario: individual, two people (same and opposite directions), Three people (same direction), and four people (same and opposite detections). Figure 3 shows the binary image for "Two People Walking in Opposite Directions" scenario. It took 4 seconds to perform the walk. The left image shows the start of the walk ( $\mathrm{t}=1$ second). Two subgroups of size 4 each (four connected neighbors) are shown. The middle image shows the subgroups at the end of the walk ( $\mathrm{t}=4$ seconds). There are three subgroups of size one, and two subgroups of size greater than one. The computational algorithm ignores these individual sensors. Hence, we got two subgroups. The Right image represents the full walk and shows the path that each person followed. Figure 4 shows the binary image for "Two People Walking in the Same Direction" scenario. It took 4 seconds to perform the walk. The left image shows the start of the walk ( $t=1$ second). Two subgroups of size 3 each (three connected neighbors) are shown. The middle image shows the subgroups at the end of the walk ( $t=4$ seconds). There were one subgroups of size one, and one subgroup of size three. If the computational algorithm ignores the individual sensors, then the middle image would show only one person. However, the speed of walking of the two persons is different and hence one finishes before the other. So, if we change the time frame we would see the second person. The Right image represents the full walk and shows the path that each 


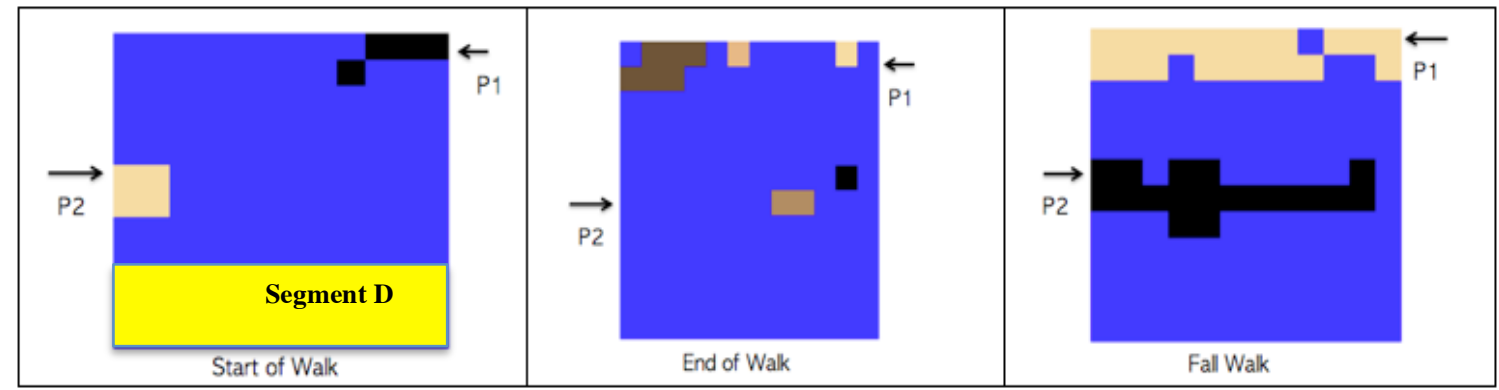

Figure 3 Active sensors map: Two people walk in Opposite directions: Longitudinal Direction $($ A <--> C), frames are grouped in a window of size 9 frames/sec (i.e. 1 second ambulation time). All sensors in Segment D are turned OFF. This applies for all experiments and results.

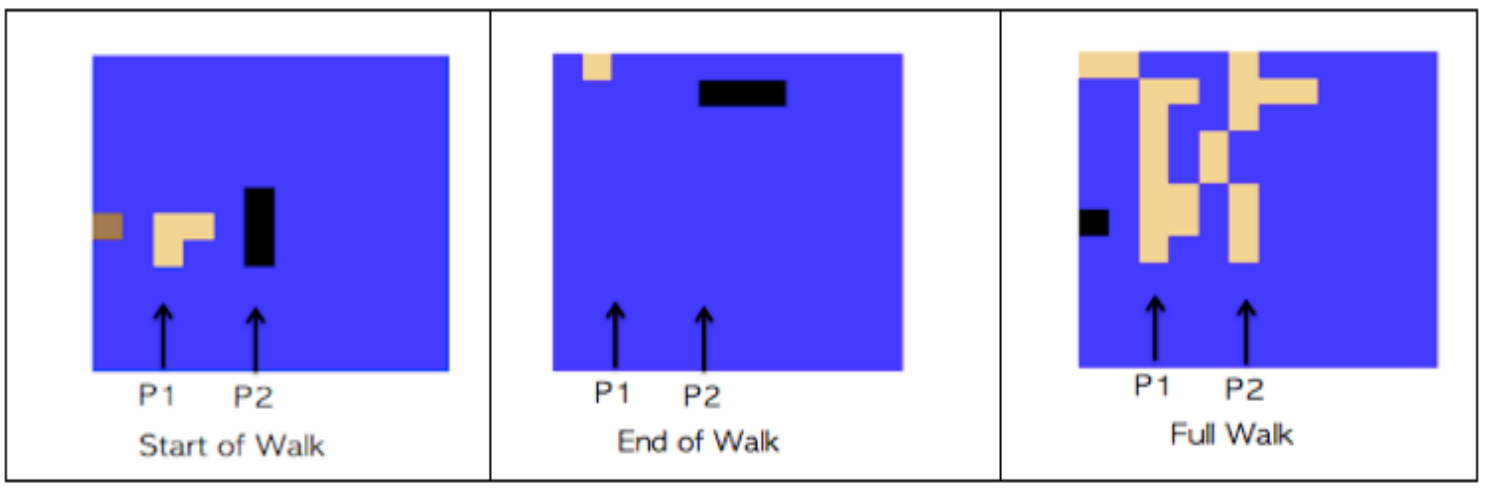

Figure 4 Active sensors map: Two people walk in the Same directions: Transverse Direction (A $\uparrow \mathrm{B}$ ), frames are grouped in a window of size 9 frames/sec (i.e. 1 second ambulation time). Segment D sensors turned OFF

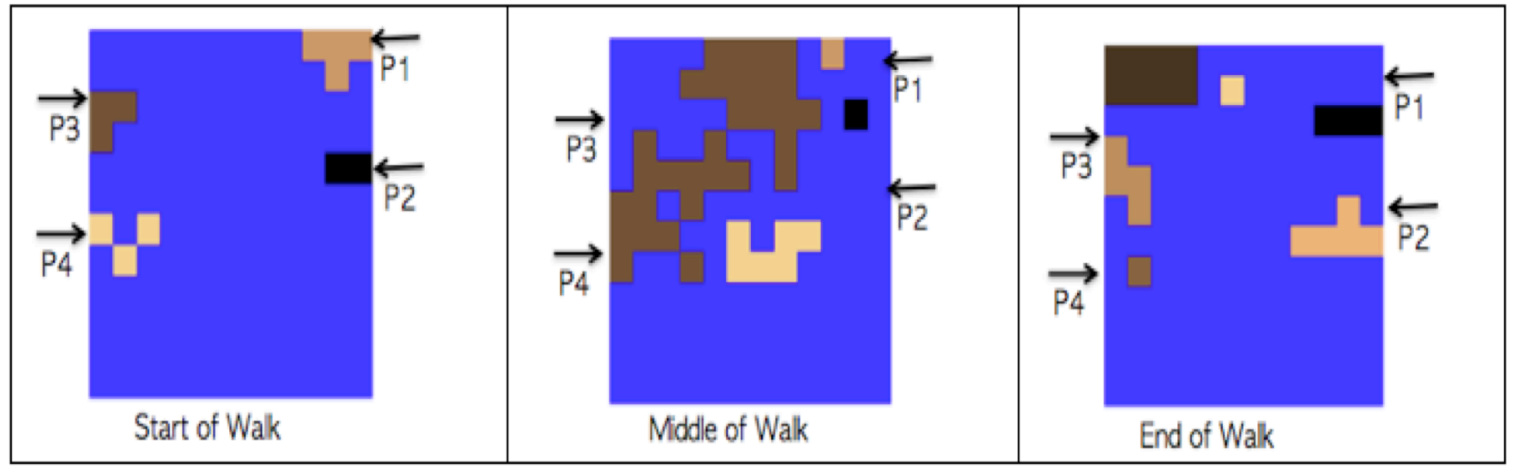

Figure 5 Active sensors map: Four people walk in Opposite directions: Longitudinal Direction (A <--> C), frames are grouped in a window of size 9 frames/sec (i.e. 1 second ambulation time). Segment D sensors turned OFF 
person was in a contiguous segment meaning the distance between the two segments is of one foot, and hence some sensors got activated due to interference. If Person 2 walks on

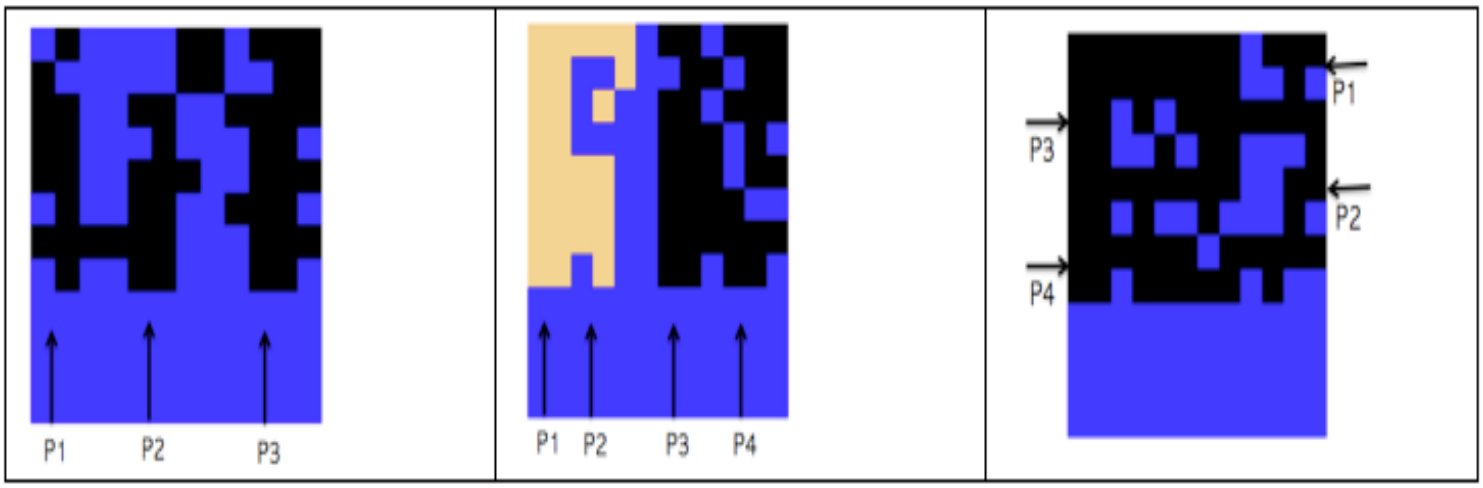

Figure 6 Active sensors map: FULL Walk Sensors distributions for: (Left) Three people walk in Same directions: Transverse Direction (A $\uparrow$ B C), (Middle) Four people walk in Same directions: Transverse Direction (A $\boldsymbol{\text { B }}$ ( C), (Right) Four people walk in Opposite directions: Longitudinal Direction $(\mathrm{A}<-->\mathrm{C})$ frames are grouped in a window of size 9 frames/sec (i.e. 1 second ambulation time). Segment D sensors turned OFF

Segment C, which is greater than five foot apart, such behavior did not exist. For example, in the opposite scenario shown in Figure 3 does not have this problem. In Figure 5 four people walk in opposite directions. At the start of the walk left the persons were at separable distance. They were recognized and by their own subgroup. However, as shown in the middle, they became closer and were not clearly separated. Then when they reached the end of the walk, right, they were separable. Figure 6 shows full image, all activated sensors during the walk, for more walk scenarios (three and four people in the same directions, and four people in the Opposite directions). We further studied one scenario for two, three and four people walking in the same direction (transverse 
direction). We ran the hybrid algorithm for different window sizes of frames. We applied the algorithm for 10 walk trials. Figure $7(\boldsymbol{a}, \boldsymbol{b}, \boldsymbol{c})$ shows the count of people for "two people walking in same direction", "three people walking in same direction", and "four people walking in same direction" scenarios for different sliding windows of frames. Results showed that we could reliably count the number of people for the "two" and "three" people scenarios. However, when number of people increased for the same size of the carpet used, it became difficult to count the people reliably (accuracy of 20\%). Accuracy is proportional the ratio of the number of people walking on the carpet and the carpet size. We could not determine the optimal window size of frames that fits all scenarios, especially when the ratio of the number of people to the carpet size is big.

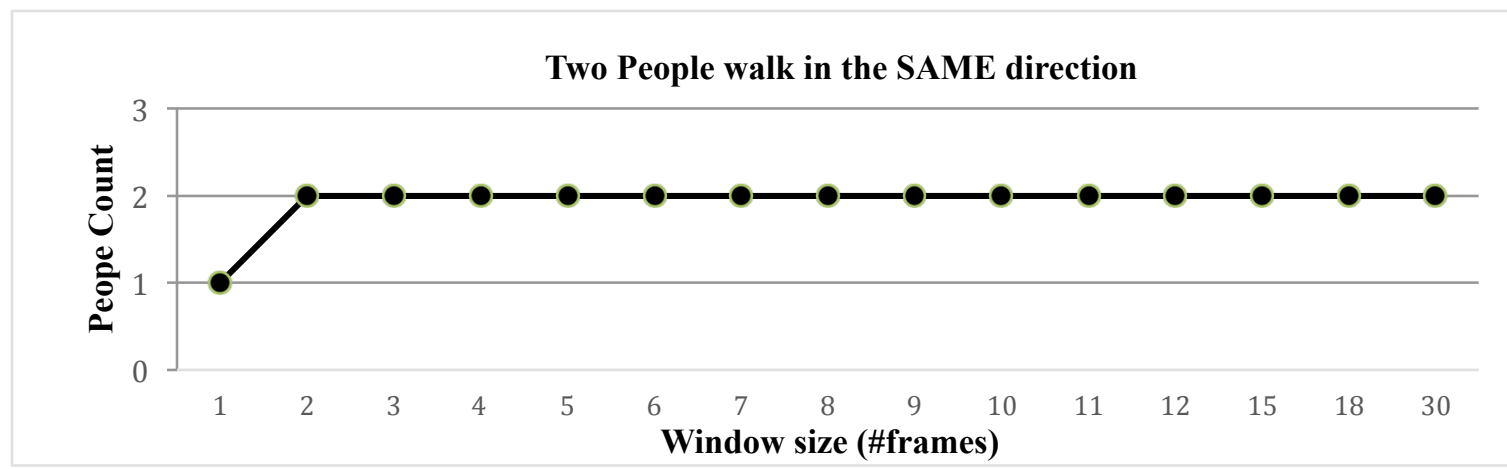

Figure 7-a People count for "two people walking in same direction" for different sliding windows of frames.

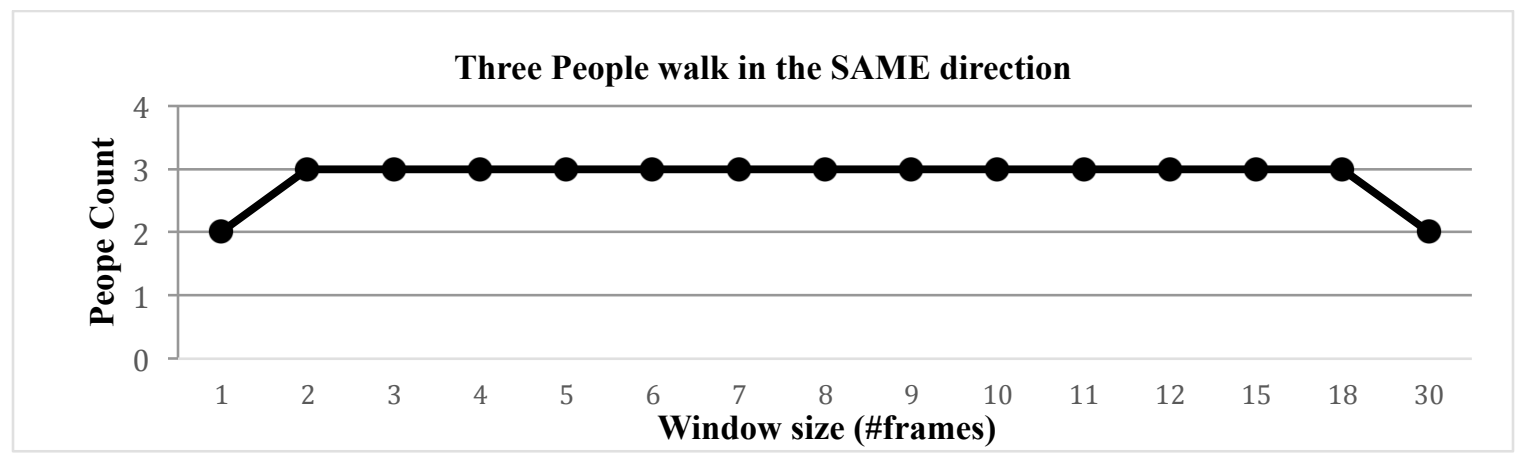

Figure 7-b People count for "three people walking in same direction" for different sliding windows of frames. 


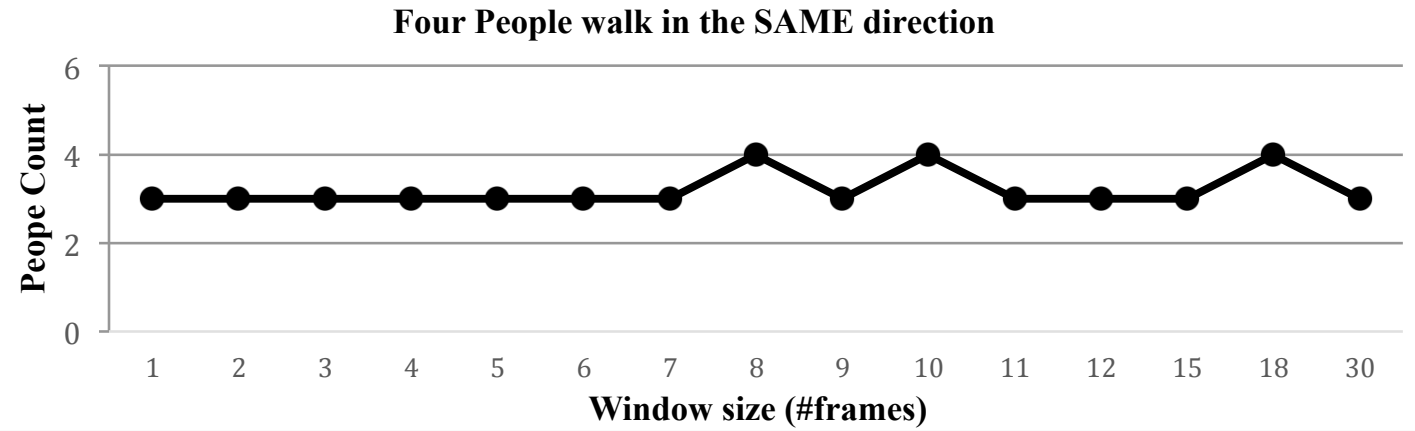

Figure 7-c People count for "Four people walking in same direction" for different sliding windows of frames.

Figure 8 shows that the count of people for two, three, and four people walking in the same direction at window size of nine ( $W S=9$ frames, i.e. the algorithm determines the count of people at time intervals of one second). As Figure 8 shows that at $W S=9$ frames, the accuracy of counting people is $100 \%$ for two people, $90 \%$ for three, and $30 \%$ for four people.

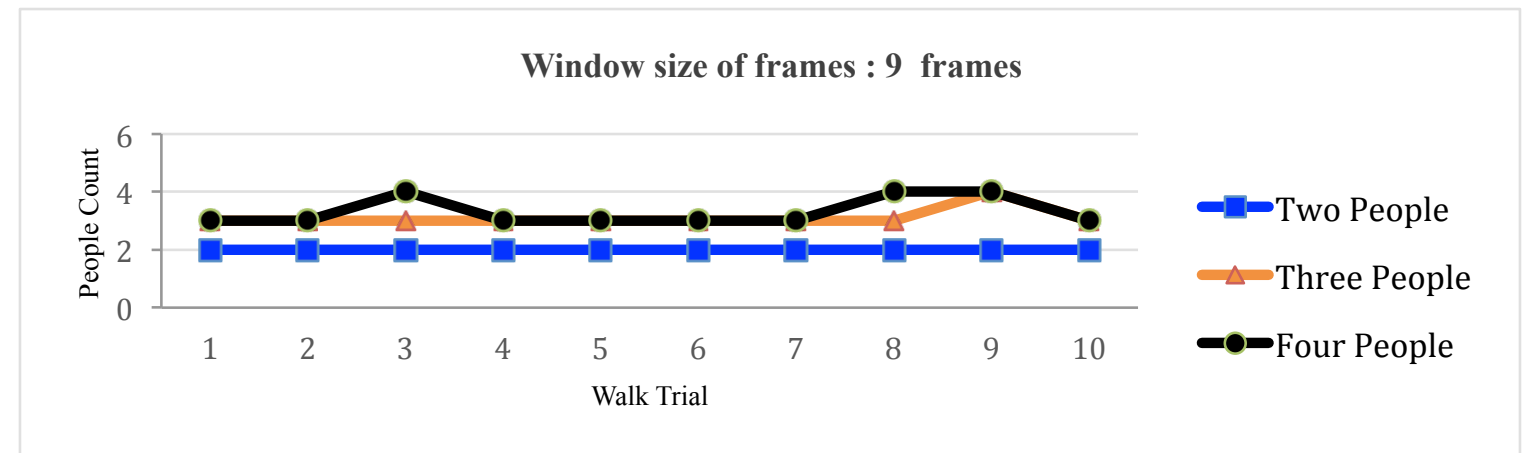

Figure 8 People count for 10 walk trials average for Windows size of 9 frames for the Two, Three, and Four people walking on the carpet 
We evaluated the binary image by the count of the ' 1 ' pixel value, which corresponds to an active sensor. Figure 9 shows the path we used to identify the average number for activated sensors for the four persons who performed the experiments.

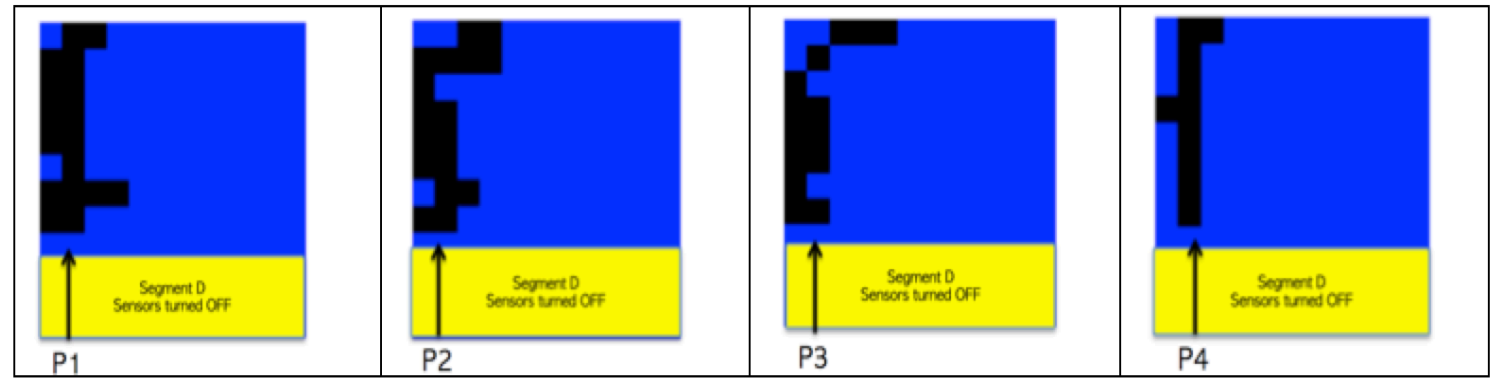

Figure 9 Individual walk trials in transverse direction. Segment D sensors turned OFF

Table II shows the activated sensors count for each scenario, and the average time it took to perform the scenario. It is clearly evident that the bigger the area is the more people walking on the carpet. Special direction. The count of the active sensors is 72 rounded to whole number. Comparing this to the 58 for the same number of people but in the same direction. The carpet layout and the time spent for the opposite directions (6 seconds) activate more sensors than and the same directions (4 seconds) of walking on the carpet. We calculated the average number of sensors activated by each walk. We then divided by the mean of active sensors produced by individual walks for the persons who performed the walk. We rounded the result to obtain the count of people. This allows us to determine the count of unknown people walking on the carpet. Table III shows the count of people using the average active sensors count, per individual(s) performing the walk trials, as the denominator for the total active sensors in the full walk trial. 
TABLE II AVERAGE ACTIVE SENSORS COUNT, AND TRAVEL TIME

\begin{tabular}{|l|c|c|}
\hline Walk Scenario & $\begin{array}{l}\text { Average active sensors } \\
\text { count-rounded }\end{array}$ & $\begin{array}{l}\text { Travel time } \\
\text { seconds }\end{array}$ \\
\hline One Person & 14 & 6.5 \\
\hline Two persons- & 27 & 4 \\
Same direction & 25 & 4 \\
\hline Two persons-Opposite direction & 49 & 4 \\
\hline Three persons -Same directions & 58 & 6 \\
\hline Four persons -Same direction & 72 & 4 \\
\hline Four persons - Opposite direction & & \\
\hline
\end{tabular}

TABLE III AVERAGE ACTIVE SENSORS COUNT, AND COUNT OF PEOPLE WALKING ON THE CARPET

\begin{tabular}{|c|c|c|c|}
\hline Walk Scenario & $\begin{array}{l}\text { Average active } \\
\text { sensors count }\end{array}$ & $\begin{array}{l}\text { Average active } \\
\text { sensors count } \\
\text { for persons' } \\
\text { walk }\end{array}$ & People Count \\
\hline One Person & 13.62 & 13.62 & 1 \\
\hline $\begin{array}{l}\text { Two persons- } \\
\text { Same direction }\end{array}$ & 27 & 14.20 & 2 \\
\hline Two persons-Opposite direction & 25 & 14.20 & 2 \\
\hline Three persons -Same directions & 49 & 14.10 & 3 \\
\hline Four persons - Same direction & 58 & 13.62 & 4 \\
\hline Four persons - Opposite direction & 48 & 13.62 & 4 \\
\hline
\end{tabular}




\subsection{Discussion and Conclusion}

In this paper, we extended the functionality of the smart carpet to count the number of people in addition to fall detection a, and gait estimation. Falls for an individual are a rare but high impact event, and affect a large fraction of the population. So, it is important to monitor, but not act until necessary. Further the system generates data $24 / 7$ and it can be recorded and processed to emphasize changes in activity as obtained by gait. Since a plurality of people walking on the floor can mimic a fall event, it is important that the system distinguish between a plurality of people and a fall event. We monitored the activity of volunteers walking on the carpet. Our algorithms were able to count the number of people at any given time with accuracy range $55 \%$ to $100 \%$. This result affected by the number of people walking, and the spatial distance that separates them. We used the total count of activated sensors compared to the average of individual walks sensors count, and were able to count the number of people with close $100 \%$ accuracy. Our results show high performance count and detection accuracy. Since our algorithms do not depend on the spatial location or dimensions of the sensors on the floor, we can count and track people on any sensors distributions. Future work will involve using the centroid in two settings; the directions of the centroid of the connected component, and, spatially, by computing the centroid of the actual dimensions and physical locations of the sensors in order to find the minimum distance by which we recognize different people. We believe more information can be deduced using this technique like gait parameters (walking speed, stride length, and step length). 


\section{References}

[1] U.S. Department of Health and Human Services: Administration on Aging, "A Profile of Older Americans: 2011,”2011.

[2] D. Giansanti, G. Maccioni and V. Macellari, The development and test of a device for the reconstruction of $3 \mathrm{D}$ position and orientation by means of a kinematic sensor assembly with rate gyroscopes and accelerometers, IEEE Trans. Biomed. Eng

[3] M. Addlesee, A. Jones, F. Livesey and F. Samaria, The ORL active floor, IEEE Personal Communications 4 (1997), 35-41. doi:10.1109/98.626980.

[4] M. Alwan, P.J. Rajendran, S. Kell, D. Mack, S. Dalal, M. Wolfe and R. Felder, A smart and passive floor-vibration based fall detector for elderly, in: 2nd IEEE Int. Conf. on Inf. \& Comm. Tech., Damascus, Syria, Apr. 24-28, Vol. 1, 2006, pp. 1003- 1007.

[5] D. Anderson, R.H. Luke, J. Keller, M. Skubic, M. Rantz and M. Aud, Linguistic summarization of activities from video for fall detection using voxel person and fuzzy logic, Computer Vision and Image Understanding 113(1) (Jan. 2009), 80-89. doi: 10.1016/j.cviu.2008.07.006.

[6] A. Sixsmith, N. Johnson and R. Whatmore, Pyrolytic IR sensor arrays for fall detection in the older population, J. Phys. IV France 128 (2005), 153-160. doi: 10.1051/jp4: 2005128024.

[7] Y. Li, K. C. Ho and M. Popescu, "A Microphone Array System for Automatic Fall Detection," in IEEE Transactions on Biomedical Engineering, vol. 59, no. 5, pp. 1291-1301, May 2012.doi: 10.1109/TBME.2012.2186449

[8] M. Popescu, Y. Li, M. Skubic and M. Rantz, An acoustic fall detector system that uses sound height information to reduce the false alarm rate, in: 30th Int. IEEE EMBS Conf, Vancouver, BC, Aug. 20-24, 2008, pp. 4628-4631

[9] E. E. Stone and M. Skubic, "Fall Detection in Homes of Older Adults Using the Microsoft Kinect," in IEEE Journal of Biomedical and Health Informatics, vol. 19, no. 1, pp. 290-301, Jan. 2015.doi: 10.1109/JBHI.2014.2312180

[10] H. Y. Lin, Y. L. Hsueh and W. N. Lie, "Abnormal Event Detection Using Microsoft Kinect in a Smart Home," 2016 International Computer Symposium (ICS), Chiayi, 2016, pp. 285-289.doi: 10.1109/ICS.2016.0064

[11] Ram M. Narayanan, Sonny Smith, and Kyle A. Gallagher, "A Multifrequency Radar System for Detecting Humans and Characterizing Human Activities for Short-Range Through-Wall and Long-Range Foliage Penetration Applications," International Journal of Microwave Science and Technology, vol. 2014, Article ID 958905, 21 pages, 2014. doi:10.1155/2014/958905

[12] Y. Li, Z. Zeng, M. Popescu and K. C. Ho, "Acoustic fall detection using a circular microphone array," 2010 Annual International Conference of the IEEE Engineering in Medicine and Biology, Buenos Aires, 2010, pp. 2242-2245.doi: 10.1109/IEMBS.2010.5627368

[13] Neelgund R., "Floor sensor development using signal scavenging for personnel detection system", University of Missouri, Columbia 2010. Master's thesis for fulfillment of MS Degree. 
[14] https://en.wikipedia.org/wiki/Connected-component_labeling. Accessed 4/4/2017

[15] https://www.mathworks.com/help/images/ref/bwlabel.html. Accessed 4/4/2017

\subsection{Further publication}

We have developed preliminary work, which is covered in a paper titled" Deriving Information from Low Spatial Resolution Floor-Based Personnel Detection System"[37], that provides the count of multiple people walking on the smart carpet. The paper was submitted to CHASE'17- The Second IEEE/ACM Conference on Connected Health: Applications, Systems and Engineering Technologies. This work is part of chapter 7. We assessed and developed algorithms to reliably determine the count of unknown number of people walking on the carpet, which will help distinguishing between a plurality of people and a fall event.

\subsection{Details and additional work not yet published}

Four people performed 10 walking sequences for different scenarios: individual, two, three and four persons walking in the same and opposite directions. We investigated the collected data, and then developed algorithms and set criteria that helped us determine the count of people walking on the carpet. In our previous paper [6], we studied thoroughly the "walking in the same direction" scenario. In this section, we extended the work to "individual, two, three and four people walking in the opposite directions". Due the spatial sampling of the carpet, and the nature of the working of the sensors, and the possible interference between sensors, we studied the data using three criteria. We used the connected component-labeling algorithm to count the number of subgroups formed 
by the activated sensors. We used the 8-connected neighborhood configuration for all experiments. We counted the number of subgroups formed regardless of their sizes to include groups of only one activated sensor. We then limit the algorithm to count the number of subgroups with greater than one activated sensor. Last; we adopted a hybrid model of both the number of subgroups formed by the neighboring activated sensors, and the count of the subgroups formed by individual activated sensor that are not direct neighbors (outside the 8 neighborhood) as described in Algorithm II. We applied these criteria for different sliding windows sizes of frames. Figure 5.10, and Figure 5.11 show

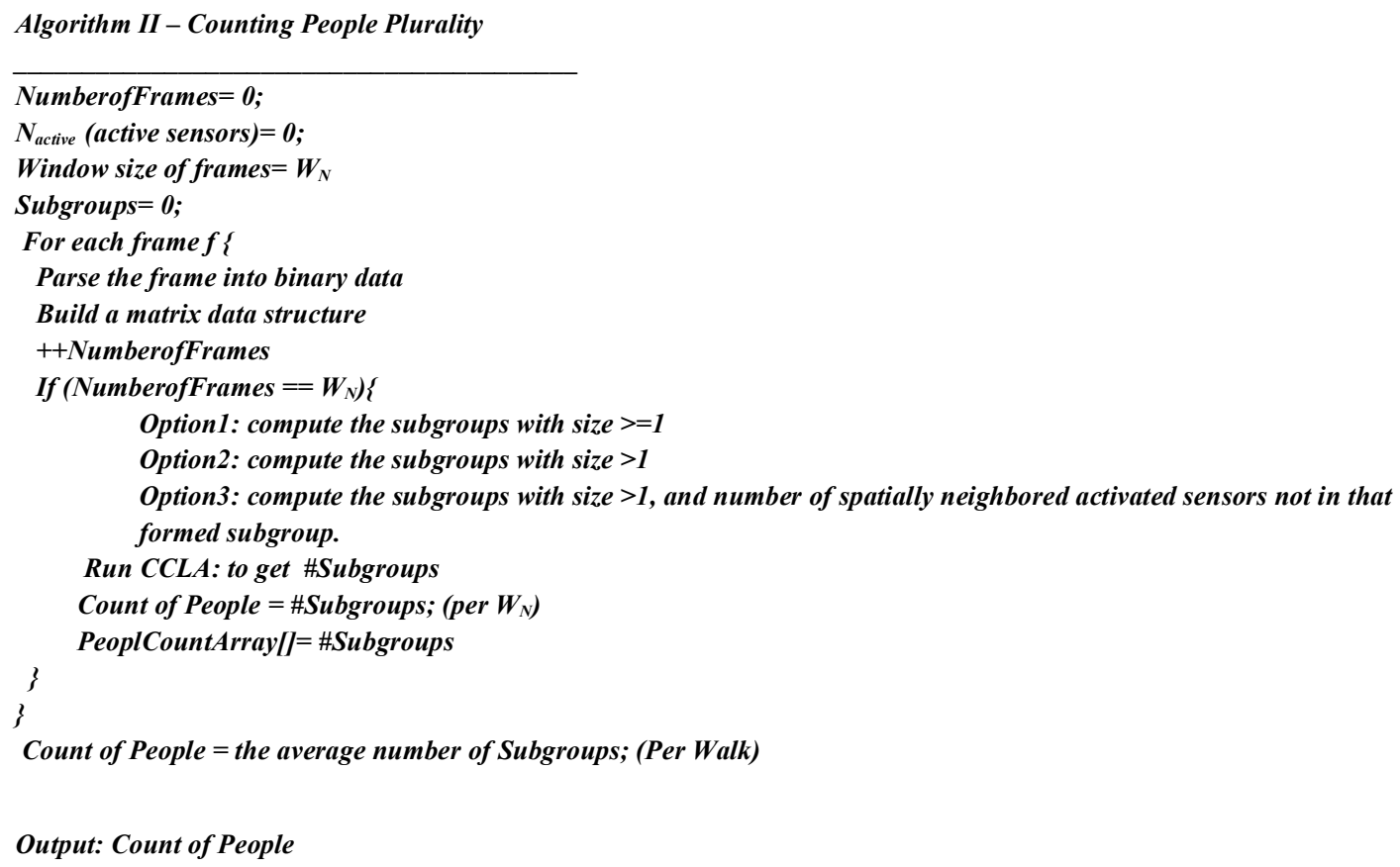

the count of subgroups formed at differing sliding window sizes (WS) for the scenario of two, three, and four people walking in the same and opposite directions. 

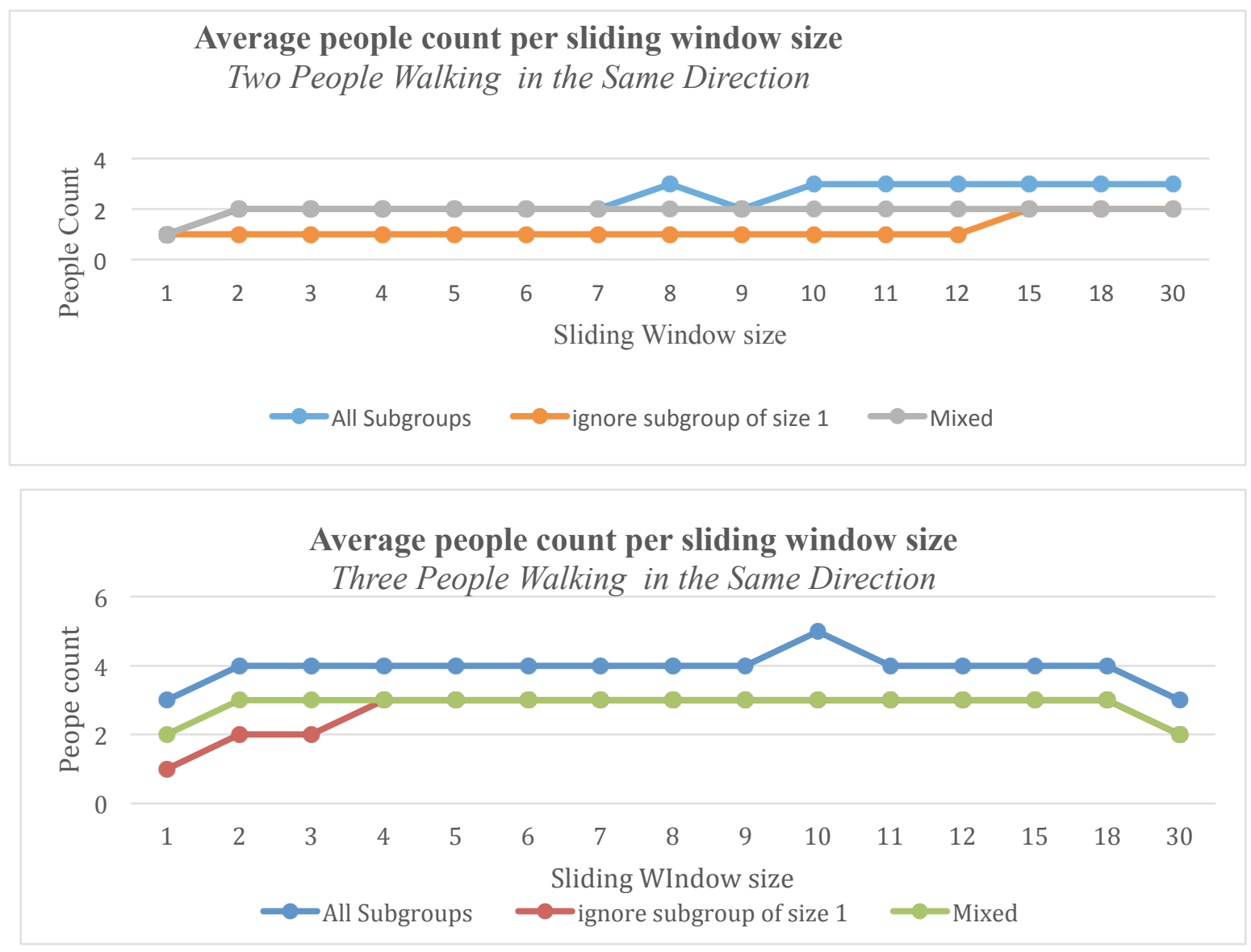

Clearly, the hybrid

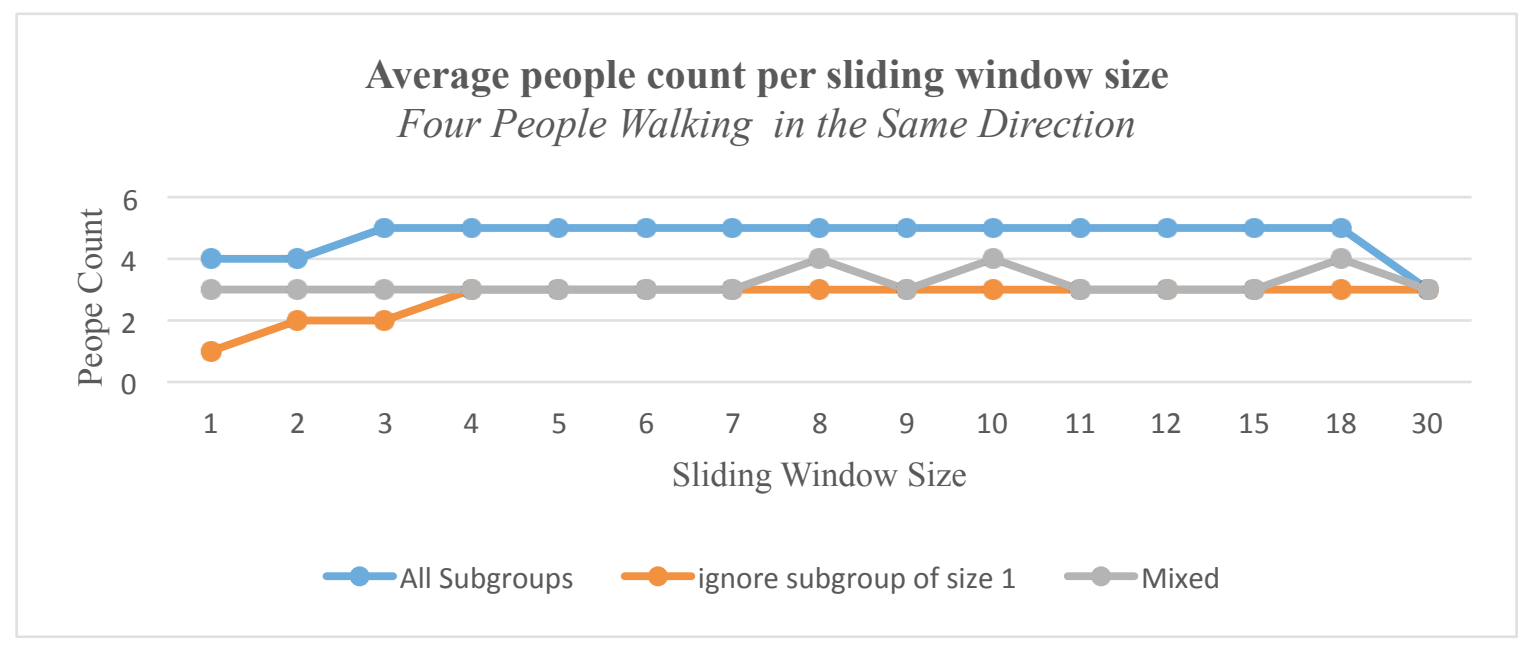

Figure 5-10 the count of subgroups formed at differing sliding window sizes (WS) for the scenario of two (top), three (middle), and four (bottom) people walking in the same direction. 

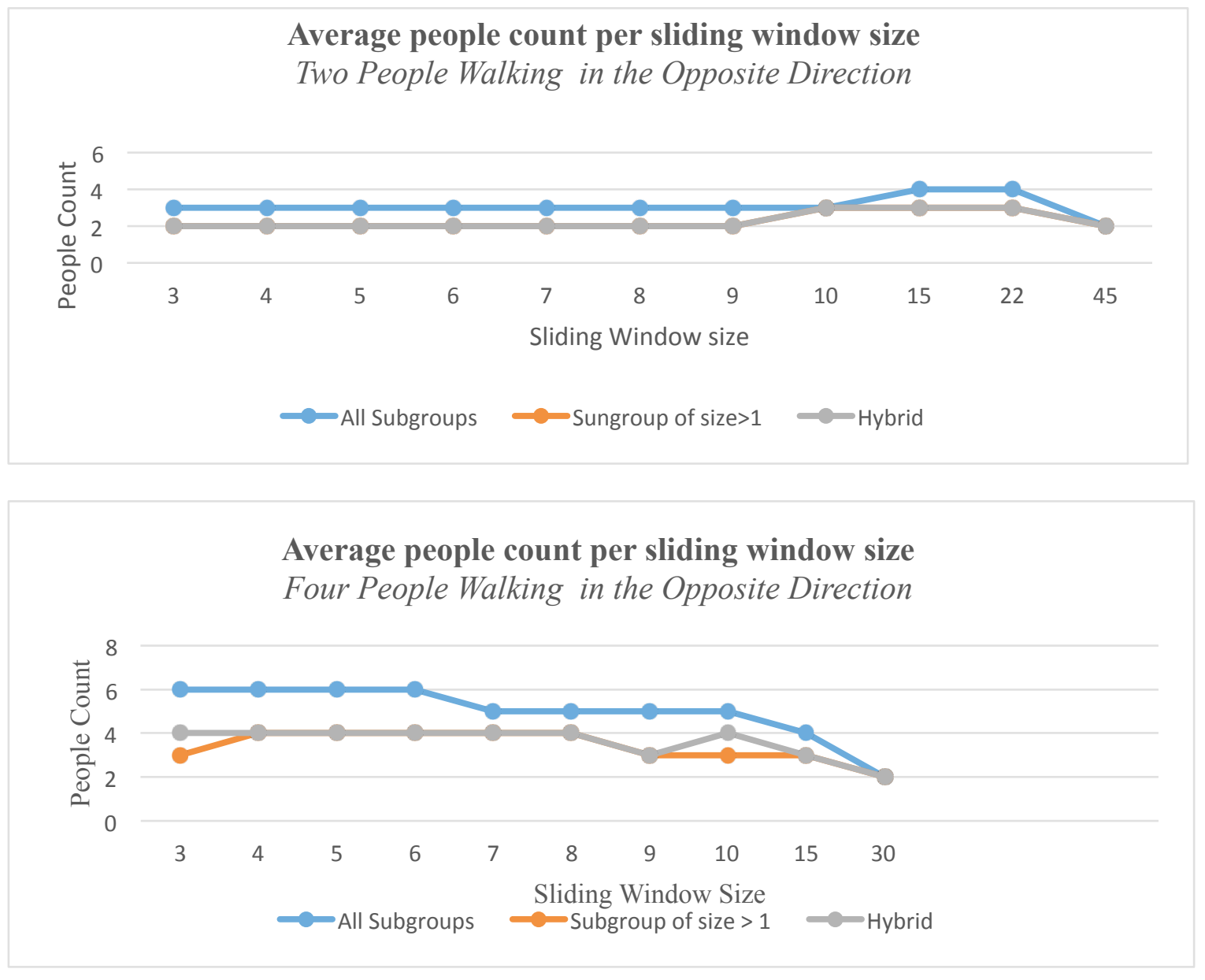

Figure 5-11 the count of subgroups formed at differing sliding window sizes (WS) for the scenario of two (top), and four (bottom) people walking in the opposite direction.

model gave consistent results, as it did not ignore the frequency of the individually activated sensors. Figure 5.12 shows the count of people for two, three, and four people walking in the same and opposite directions at window size of nine ( $W S=9$ frames, i.e. the algorithm determines the count of people at time intervals of one second). As Figure 5.12 shows that at $W S=9$ frames, the accuracy of counting people is $100 \%$ for two people same direction and $60 \%$ for opposite direction, $90 \%$ for three in the same direction, and $30 \%$ for four people in the same direction, and $20 \%$ for four people in opposite directions. The more people walk on a fixed size carpet, the lower the accuracy to count 
them. In addition, changing the sliding window size per scenario would achieve better results. For example Four People in the opposite direction, achieved better accuracy $(70 \%)$ for $W S=7$, and $W S=5)$.

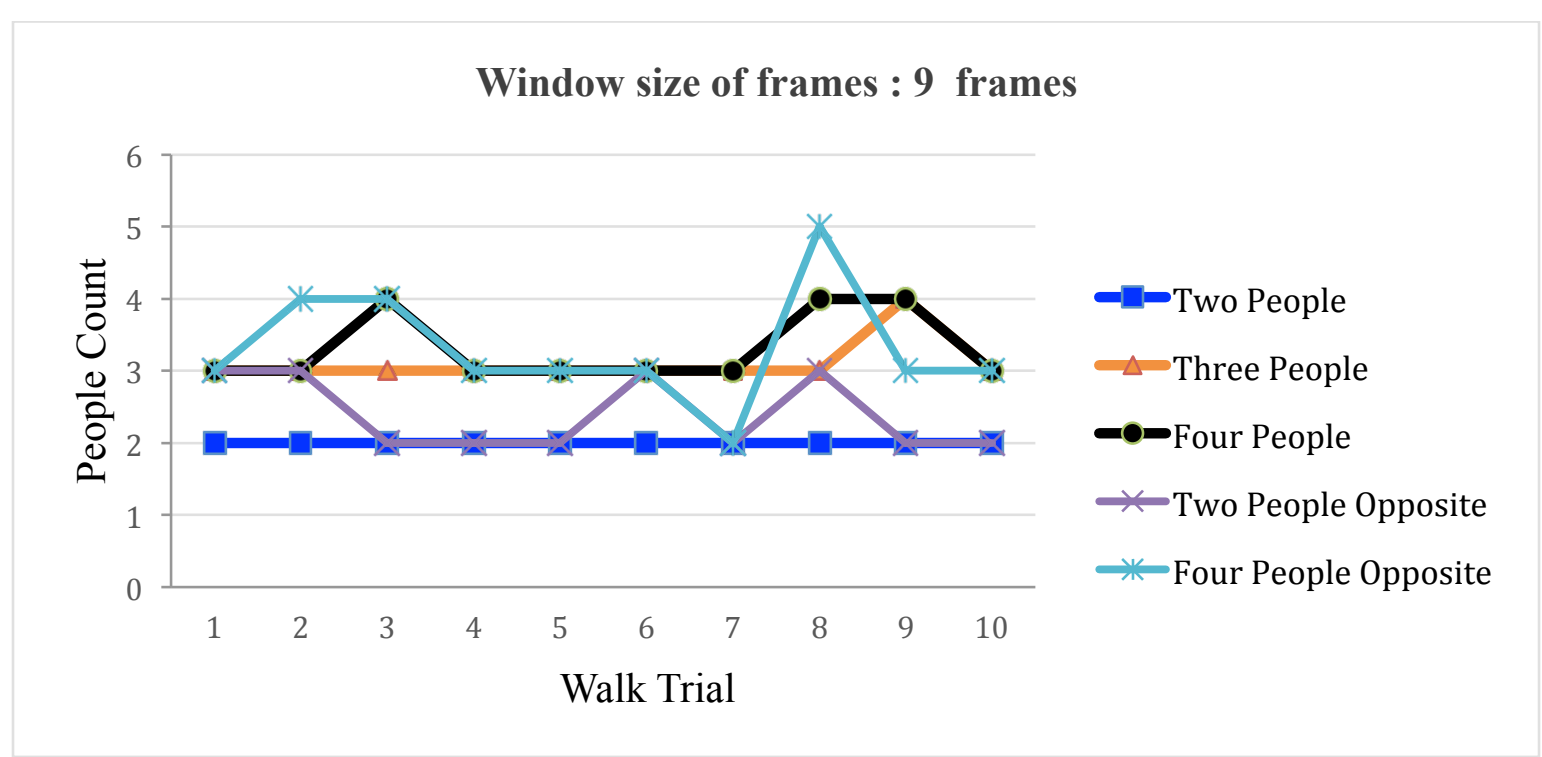

Figure 5-12 the count of people for two, three, and four people walking in the same and opposite directions at window size of nine (WS $=9$ frames, i.e. the algorithm determines the count of people at time intervals of one second).

We looked at the data as it progress, as a time series, and then evaluated the count of number of people. We combined the nine walk trials performed by subject 1 (Adult male) into one time series, and we ran the algorithm for the counting total number of subgroups using the mixed (hybrid) model per different window sizes. Figures 5.13- 17 shows the result for different sliding window sizes. 

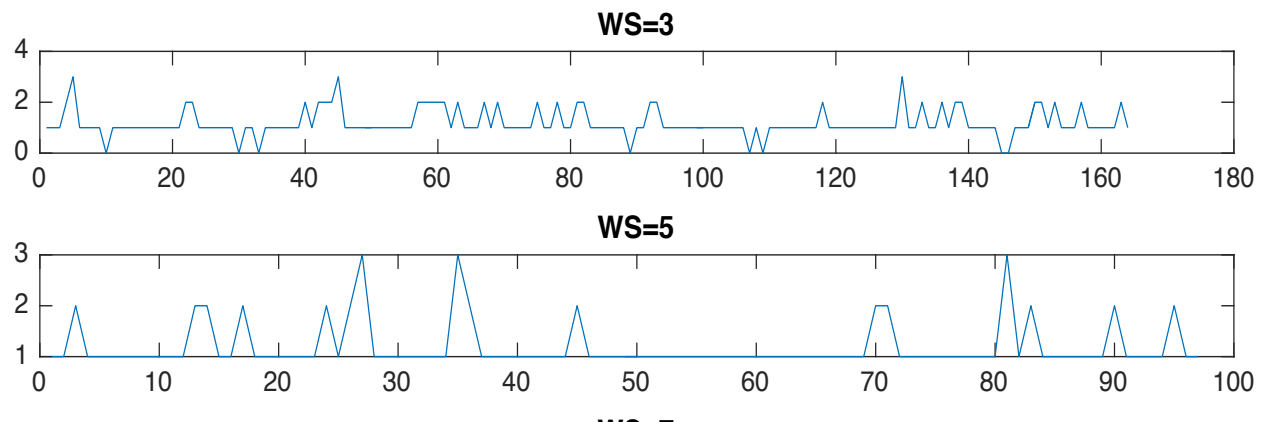

WS=7
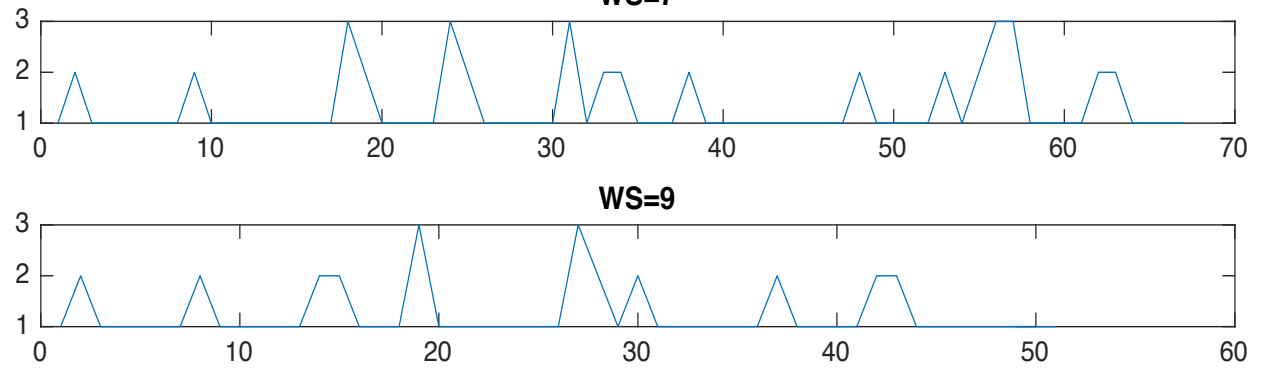

WS=12

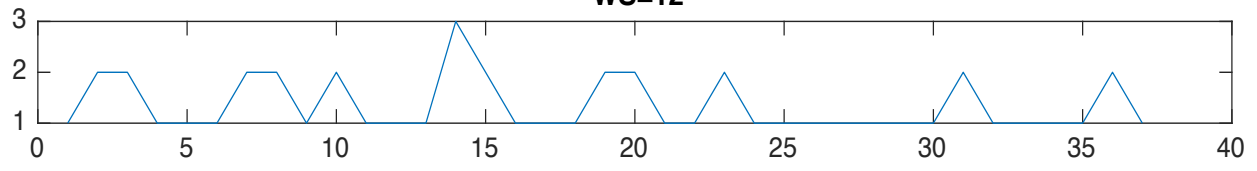

WS $=15$

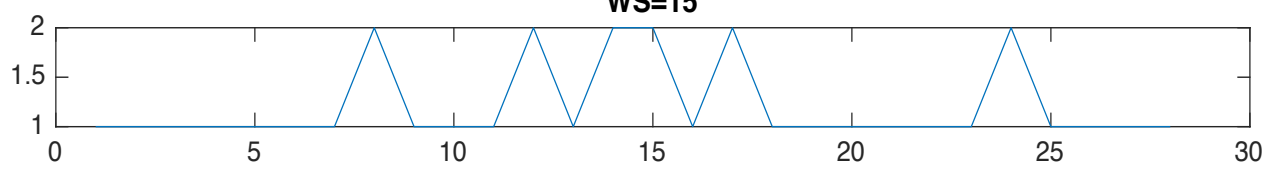

WS $=30$

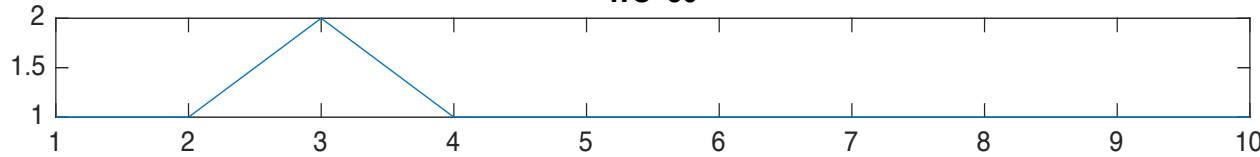

Figure 5-13 The number of subgroups formed by a participant walking alone on the carpet. 


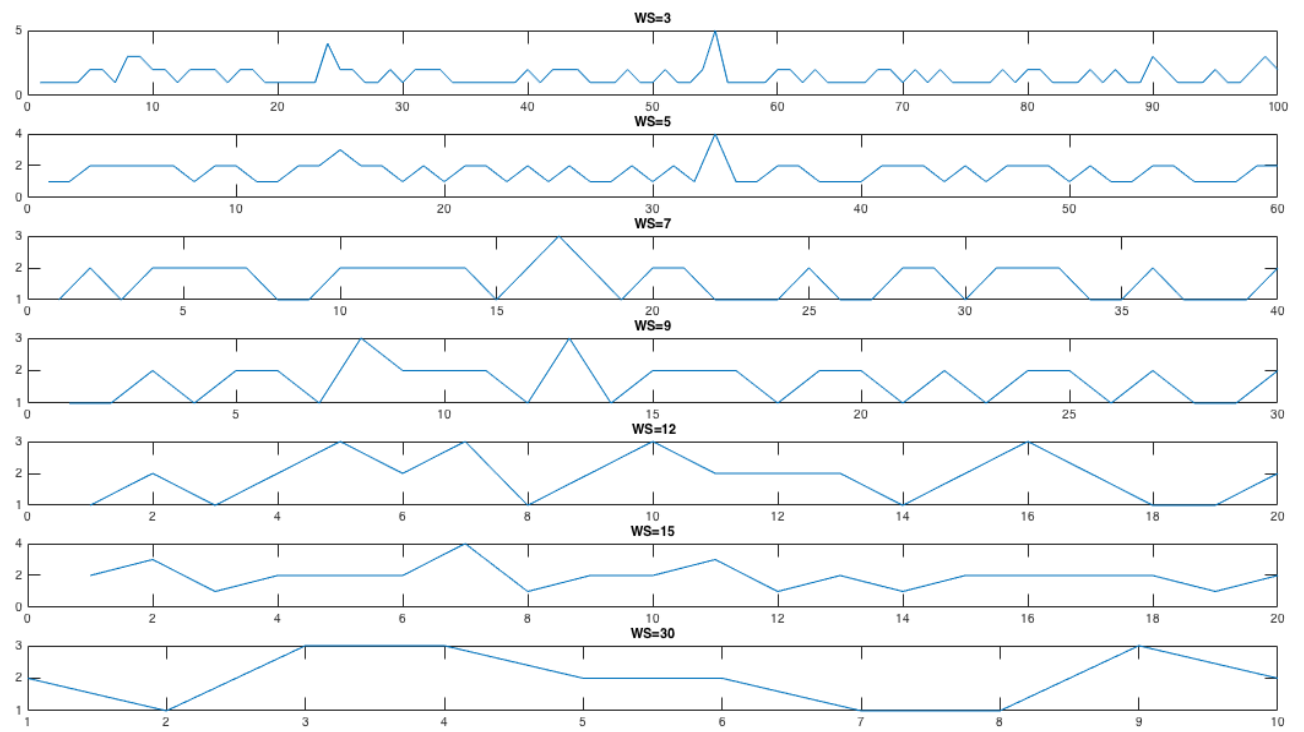

Figure 5-14 The number of subgroups formed by two participants walking in the same direction
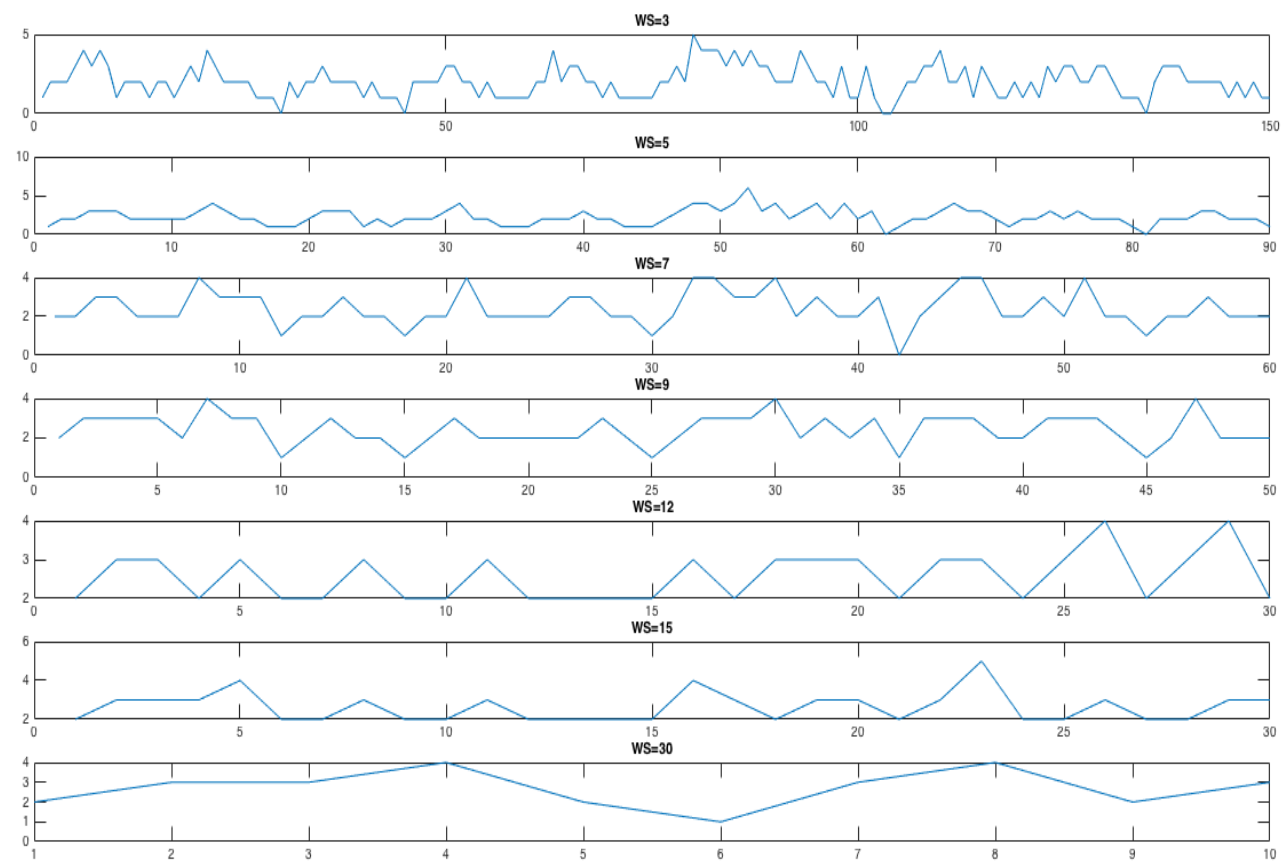

Figure 5-15 The number of subgroups formed by two participants walking in the opposite direction 


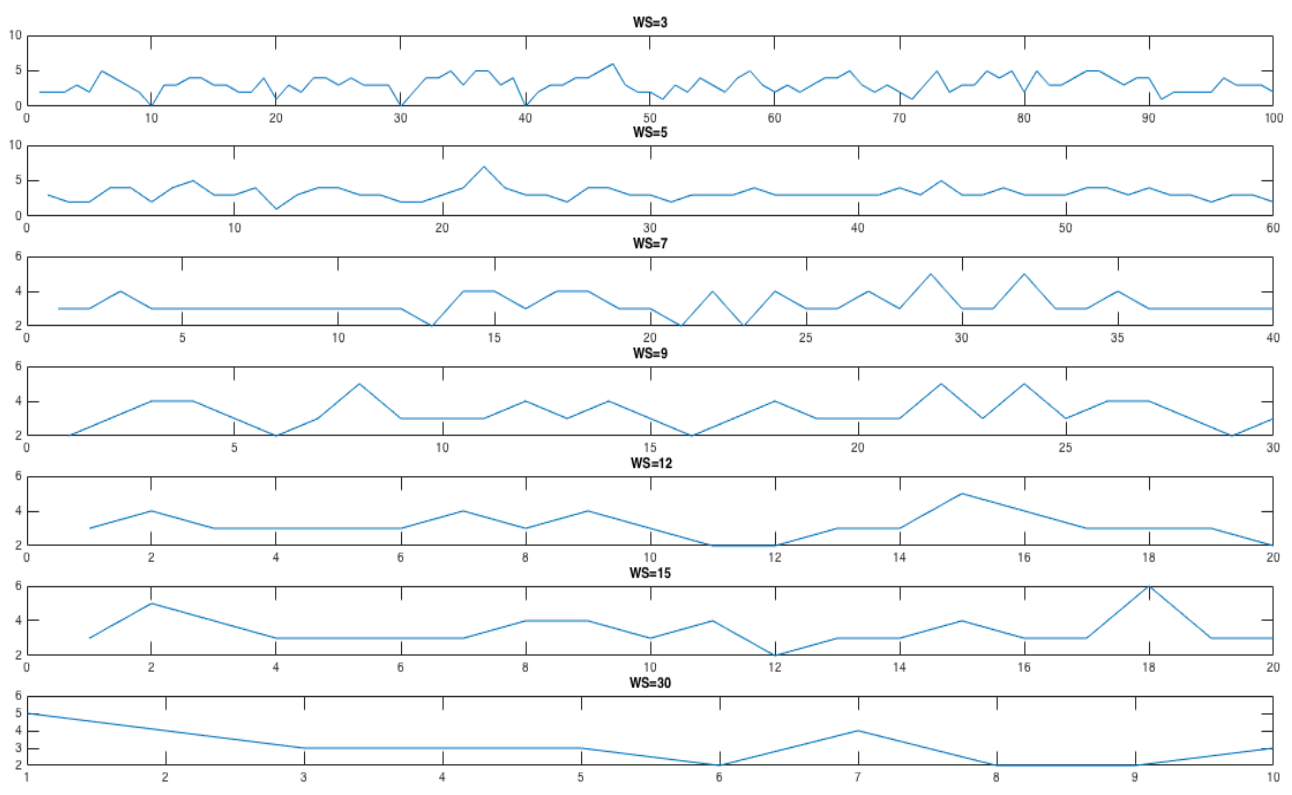

Figure 5-16 The number of subgroups formed by four participants walking in the same direction
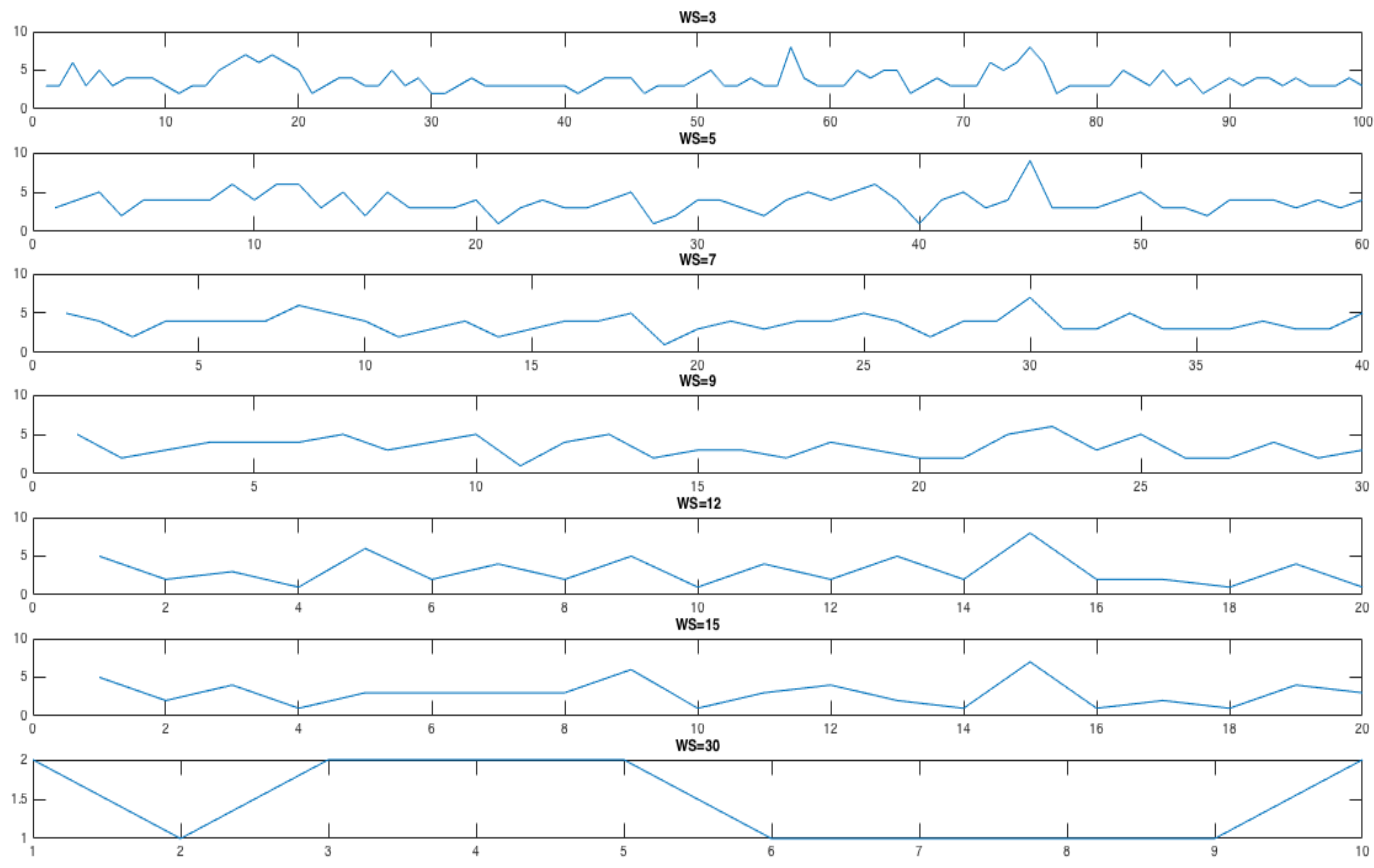

Figure 5.17 The number of subgroups formed by four participants walking in the opposite direction 


\subsection{Conclusion}

We monitored the activity of volunteers walking on the carpet for different walking scenarios. Our algorithms were able to count the number of people at any given time, and at the best we did was constantly have an average accuracy of $100 \%$. The number of people walking, the spatial distance that separates the people, and the ratio of the number of people walking to the actual carpet size influenced the results. We found that the bigger the ratio the lower the counting accuracy. For each scenario, we used the total count of activated sensors, but also the average count of activated walk sensors. Our results show high performance count and detection accuracy. Since our algorithms do not depend on the spatial location or dimensions of the sensors on the floor, we can count and track people on any sensors distribution. 


\section{CHAPTER 6: ADDITIONAL INTELLIGENCE, GAIT ESTIMATION, AND SCAVENGING CHARGING CHARACTERISTICS}

\subsection{Introduction}

We produced a paper titled" Floor Based Sensor System: Additional Intelligence, Gait Estimation, and Scavenging Charging Characteristics"[5], which describes additional fall detection algorithms, statistics and correlation between the GAITRite mat and smart carpet systems, and smart carpet sensors scavenged signal. The paper was submitted to ICOMP'17The 18th International Conference on Internet Computing and Internet of Things. In this paper we expanded our work on gait analysis to measure how much the results we obtained agrees with the gold standard, the GAITRite. In addition, we looked at the historical data collected from previous work $[1,3]$ and evaluated new fall detection algorithms. We shared our initial work and thought about the scavenged signal characteristics and the value of studying it in the context of profiling people walking on the smart carpet. 


\title{
6.2 Manuscript: Floor Based Sensor System: Additional Intelligence, Gait Estimation, and Scavenging Charging Characteristics
}

\author{
Fadi Muheidat and Harry W. Tyrer, \\ Electrical and Computer Engineering department, University of Missouri, Columbia, USA
}

\begin{abstract}
In this paper we propose further in-depth analysis to our smart carpet, a floorbased personnel detecting system. We have added more intelligence by enhancing fall detection algorithms. Both a convex hull, and heuristic algorithms were developed to detect falls. The proposed algorithms detected fall with $95 \%$ sensitivity and $85 \%$ specificity when combining both methods exclusively. We extracted and estimated gait parameters, comparing our system to the GAITRite system, which is used as gold standard; here we investigate whether the differences between the two systems are statistically significant. The Statistical T-Test showed excellent agreement between the smart carpet and the GAITRite in estimating gait parameters. With $(\mathrm{P}=0.55)$, the walking speed differences of the two systems are not statistically significant. Additionally, we studied the characteristics and the behavior of the sensor's scavenged signal. We designed and built a single large sensor, where subjects performed multiple walks on the sensor, and their data recorded and studied. The sensors' voltage waveforms behaved differently corresponding to different people and set of walking trials. The covering material, and the environmental conditions affect the behavior of the scavenged signal. More detailed study and experimental trials are needed.
\end{abstract}

Keywords: Signal Scavenging, Fall Detection, Gait Estimation, Sensor, and Eldercare 


\section{2 $\$ 1.Introduction}

Research focused on older adults continues to promote successful aging, especially regarding how to enhance the overall quality of life and provide adequate medical care while keeping health care cost under control. Technology is a welcome addition to the population of the elderly; it offers the elderly full productive and independent lives [1].

The continued increase in longevity will yield a steep rise in the old-age dependency ratio, defined as the ratio of the number of elderly people to those of working age. This ratio is expected to double from $11.7 \%$ to $25.4 \%$ in the next 35 years [2]. The number of people aged 80 and over is going to triple in the next 35 years [3]. Approximately 28$35 \%$ of people aged 65 and over fall each year increasing to $32-42 \%$ for those over 70 years of age [4]. Severe fall injuries can also lead to deaths [5]. Several studies have shown that better outcomes are correlated with rapid initiation of medical intervention immediately after a fall [6].

Research, then, to develop new technology or enhance existing ones to detect falls and can help reduce the consequences of a fall. All fall detection systems have a common objective, to distinguish a fall from activities of daily living, which tends not to be an easy problem to solve. However, falls to an individual is a rare event even though the elder population fall frequency is high. Fall prediction or fall risk analysis extends the functionality of smart carpet by extracting and estimating Gait Parameters [7]. Recent research shows that change in gait parameters may be predictive of future falls and adverse events in older adults such as physical functional decline [8-11] and fall risks [12-14]. 
Privacy is major concern, so there is a need for context-aware sensor systems that passively detect human presence or activities. Multiple passive systems were developed to detect falls [15-21]. Each of these systems has limitations and/or complex implementation, and high cost. Our system, the smart carpet, is designed to scavenge the signal from the environment. The sensor made from a conductive material picks up stray $60 \mathrm{~Hz}$ noise to detect presence of the person without much complexity [22-25].

This paper describes development in fall detection algorithms, analysis of the gait estimating system, and study of the charging characteristics of the scavenged signal. Section 2 of this paper provides an overview of the system, and our methodology. Section 3 contains the experimental results. Finally, the major points of this paper are discussed along with future work.

\section{2\2. Methodology}

\section{a. System Overview}

Our system, as shown in Figure 1, consists of the Smart Carpet, which is a floor based personnel detector, data acquisition system, and processor. The signal scavenging sensors can be built and produced in different sizes and shapes. All sensors function the same. The sensors are connected to data acquisition system that scans at configurable speeds depending on the size and number of sensors in one segment. Signals converted into digital values with a constant threshold using 10-bit Analog to Digital convertor, and then read by a computer as an ASCII frame. The software components process the data frames, and use different computational intelligence methods to perform the required operations like fall detection, gait estimation, data visualization, and notification. 
Additionally, the system can show the signal data scavenged by the sensor for fine-tuning of the system parameters.
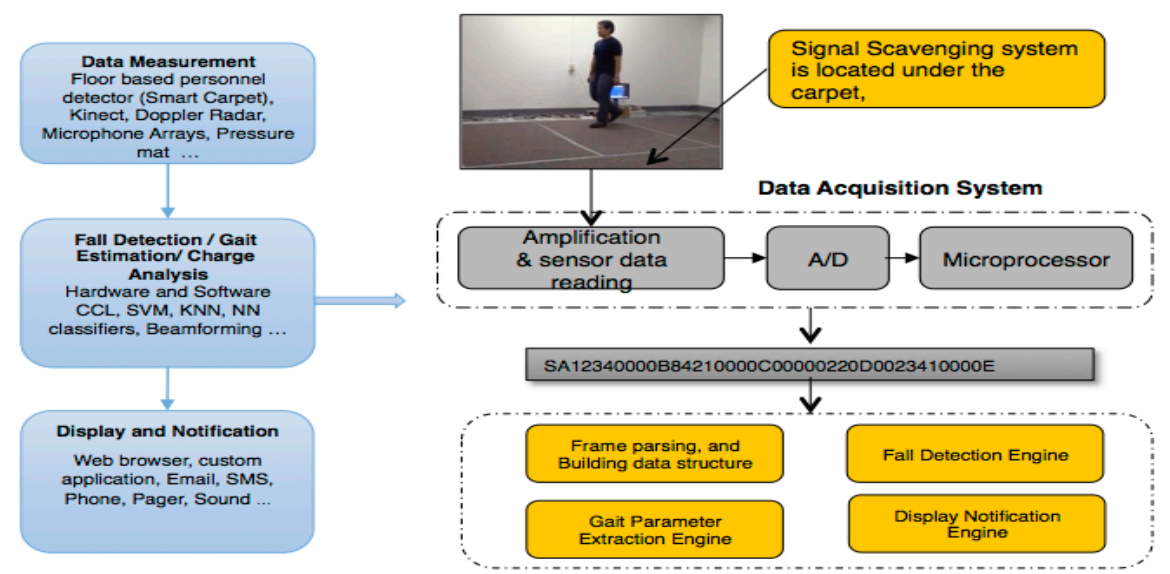

Figure 1 System overview

\section{b. Fall Detection}

In order to detect falls, we need to detect motion on the carpet. To achieve this goal, we built sensors and organized them into 4 segments with 32 sensors each. The data acquisition system in Figure 1 reads a total of 128 sensors. After a person walks or has mobility on the carpet, the voltage generated by the sensors that exceeds certain threshold, determined experimentally, is considered active. As more motion occurs on the carpet more sensors become active. In this experiment, we have 10 volunteers each of them performed 8 walk-fall patterns. The falling patterns adapted from previous work by our eldercare technology research group [26]. Figure 2 shows the walk-fall pattern. Each rectangle represents a set of frames (each frame resulted from one complete scan of the 128 sensors). Recorded videos used as gold standard to verify the accuracy of the fall 
detection algorithms. In [25] we used different algorithms and sliding window with different sizes $(W S)$ sizes to determine the fall. Later, the data used to train a classifier and add intelligence to the system. In this paper, we will discuss two algorithms developed and enhanced to help detect falls; convex hull area, and active sensors count given certain active sensor layout; we call the later method "Heuristics".

\begin{tabular}{|c|c|c|c|c|c|c|c|c|}
\hline Walk & Walk & Walk & Walk & Walk & Walk & Walk & Walk & Walk \\
\hline Walk & & & & & & & & \\
\hline Walk & Walk & Walk & Walk & Fall & & & & \\
\hline
\end{tabular}

Figure 2 Walk-Fall pattern: Each rectangle represents one frame

\section{b.1 Convex Hull Method}

In convex hull area algorithm, we used a window size of number of frames (sliding window of size $W S$ ) to form an array list of active sensors, and then apply the quick convex hull algorithm [27]. We found the points forming the convex hull (polygon) for the set of active sensors on the carpet. We calculated the area of the polygon according the shoelace algorithm [28]. To detect a fall, we run the algorithm for different window sizes $(W S)$ and thresholds $(T H)$. In Figure 2 above each colored cell represents a sliding window of size $W S ; W S=1,2,3,4$...etc. So, we group the active sensors and then apply the algorithm. Having a constant Threshold (TH) with changing (WS) didn't give good result. Our approach is to make the threshold variable based on the number of active sensors forming the hull. The new threshold is given by the equation:

$$
T H=W S * H S * \alpha_{H U L L}
$$

WS: the sliding window size,

$\boldsymbol{H S}$ : number of points forming the convex in a $W S$.

$\boldsymbol{\alpha}_{\boldsymbol{H U L L}}:$ constant determined experimentally $=0.3$ 


\section{b.2 Heuristic Method}

In heuristic algorithm, we converted the array of sensors data into a matrix format. Then we counted the number of active sensors that are contiguous, as shown in Figure 3 in a row or column. We determine the fall if the count meets or exceeds some threshold $(T H)$. The Threshold is given by the equation:

$T H=W S * \alpha_{\text {Heuristic }}$

$\boldsymbol{W S}$ : the sliding window size,

$\boldsymbol{\alpha}_{\text {Heuristic }}:$ constant determined experimentally $=1.2$

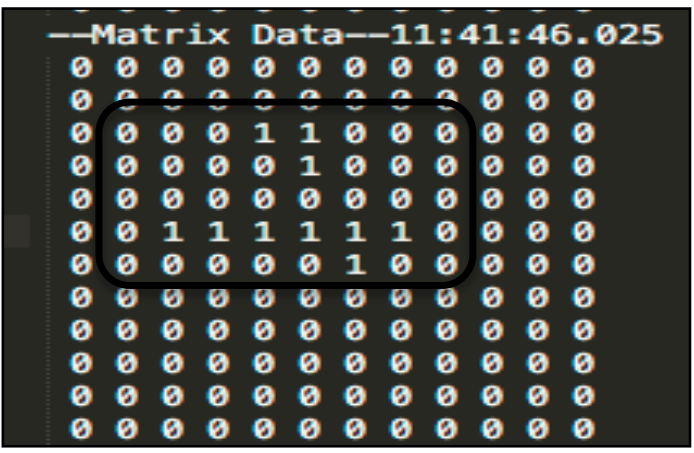

\begin{tabular}{|llllllllllll}
\hline$-M$ & Mat & $i x$ & Data & -1 & 7 & 5 & 0 & 3 & 35 & 33 \\
1 & 0 & 1 & 0 & 0 & 0 & 0 & 0 & 0 & 0 & 0 & 0 \\
0 & 0 & 1 & 1 & 0 & 0 & 0 & 0 & 0 & 0 & 0 & 0 \\
1 & 1 & 1 & 1 & 1 & 1 & 1 & 0 & 0 & 0 & 0 & 0 \\
1 & 1 & 1 & 0 & 1 & 1 & 1 & 0 & 0 & 0 & 0 & 0 \\
0 & 0 & 1 & 1 & 0 & 0 & 0 & 0 & 0 & 0 & 0 & 0 \\
1 & 0 & 0 & 1 & 0 & 0 & 0 & 0 & 0 & 0 & 0 & 0 \\
0 & 0 & 1 & 1 & 0 & 0 & 0 & 0 & 0 & 0 & 0 & 0 \\
0 & 0 & 0 & 1 & 0 & 0 & 0 & 0 & 0 & 0 & 0 & 0 \\
0 & 0 & 0 & 0 & 0 & 0 & 0 & 0 & 0 & 0 & 0 & 0 \\
0 & 0 & 0 & 0 & 0 & 0 & 0 & 0 & 0 & 0 & 0 & 0 \\
0 & 0 & 0 & 0 & 0 & 0 & 0 & 0 & 0 & 0 & 0 & 0 \\
0 & 0 & 0 & 0 & 0 & 0 & 0 & 0 & 0 & 0 & 0 & 0 \\
\hline
\end{tabular}

Figure 3 Data Matrix samples for Heuristic algorithm

\section{c. Gait Estimation Performance}

In previous work [6], we used the smart carpet to estimate the gait parameters of walking speed, stride time, and stride length. We measured the distance travelled, and elapsed time. We computed the number of footfalls. We used these parameters to estimate the gait characteristics of the persons walking on the carpet. Results showed that we could extract and estimate the parameters with acceptable relative error within acceptable standard deviation. Nine subjects participated in the experiment. Each subject was tested multiple times, trying to maintain same walk pace. In total, there are 75 walk 
sequences. Subjects normally take about 5-10 steps to complete the walkway. In this paper, we are interested in measuring how much our system matches the GAITRite; the gold standard for gait measurements. Table I shows the mean, median, standard deviation and standard error for walking speed, stride time, and stride length of the data resulted from the 75 walk sequence. Figure 4 shows the histogram of walking speed left (top, and bottom), stride length middle (top, and bottom), and stride time right (top, and bottom), fitted with normal distribution function.
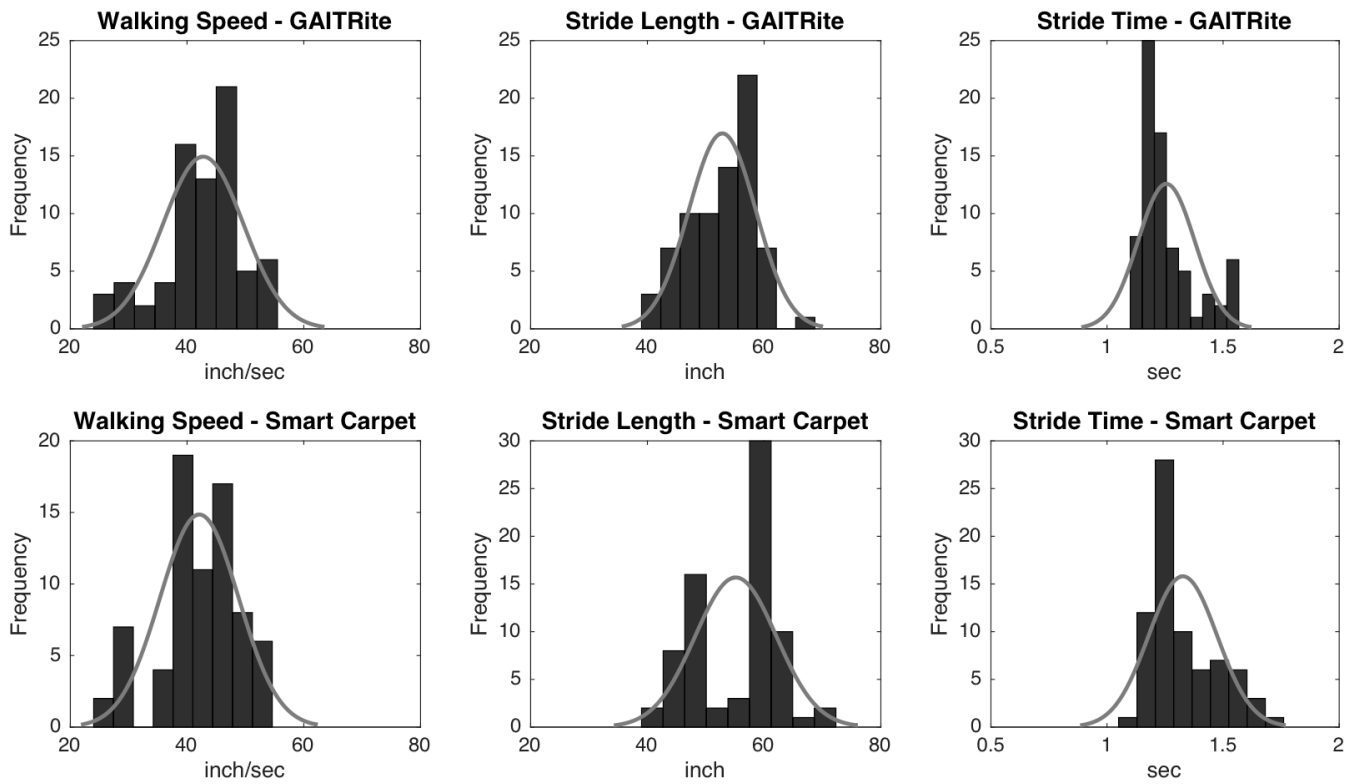

Figure 4 histogram of walking speed, stride length, and stride time fitted with normal distribution function 
TABle I MEAN, MEDian, STANDARD DEVIATION, AND STANDARD ERROR FOR 75 WALK SEQUENCE

\begin{tabular}{|c|l|l|c|c|}
\hline & Mean & Median & $\begin{array}{l}\text { Standard } \\
\text { Deviation }\end{array}$ & $\begin{array}{l}\text { Standard } \\
\text { Error }\end{array}$ \\
\hline \multicolumn{5}{|c|}{ Walking Speed } \\
\hline GAITRite & 42.7666 & 43.1299 & 6.9181 & 0.804 \\
\hline Carpet & 42.0995 & 42.6457 & 6.7559 & 0.785 \\
\hline \multicolumn{5}{|c|}{ Stride Time } \\
\hline GAITRite & 1.2557 & 1.22 & 0.1221 & 0.014 \\
\hline Carpet & 1.3268 & 1.27 & 0.1475 & 0.017 \\
\hline & & 54.9075 & 5.7459 & 0.668 \\
\hline GAITRite & 52.8381 & 58 & 6.9634 & 0.809 \\
\hline Carpet & 55.1429 & 58 &
\end{tabular}

We used different statistical tests to measure how well the two systems match, and whether the differences between the two systems are statistically significant or not statistically significant. Using the two-sample t-test (ttest2), a t-test returns a test decision for the null hypothesis that the data in GAITRite and smart carpet comes from samples from normal distributions with equal means [29]. The returned value of $\mathbf{H}_{\mathbf{0}}=0$ indicates that ttest2 does not reject the null hypothesis at the default $5 \%$ significance level. The alternative hypothesis is that the data in GAITRite and smart carpet comes from samples with unequal means. The result $\mathbf{H}_{0}=\mathbf{1}$ if the test rejects the null hypothesis at the $5 \%$ significance level, and 0 otherwise. The $p$-value is the probability of observing a test 
statistic as extreme as, or more extreme than, the observed value under the null hypothesis. Small values of $p$ cast doubt on the validity of the null hypothesis. A MannWhitney U-test, also called the Wilcoxon rank sum test, is a nonparametric test that compares two unpaired groups. It ranks all the values from low to high, paying no attention to which group each value belongs. The smallest number gets a rank of 1 . The largest number gets a rank of $N$, where $N$ is the total number of values in the two groups. If the means of the ranks in the two groups are very different, the $p$-value will be small. It tests the null hypothesis that data in GAITRite and smart carpet are samples from continuous distributions with equal medians, against the alternative that they are not. Additionally, we calculated strength of linear correlation, and fluctuations of one of the system with respect to the other by computing the correlation coefficients, and the coefficient of determination (r-squared) respectively for Walking Speed, Stride Time and Stride length [31].

\section{d. Signal Scavenging Charging Characteristics}

Signal scavenging originated from the concept of energy harvesting from the existing energy in the environment. Our sensor system is scavenging energy from $60 \mathrm{~Hz}$ stray electromagnetic waves. When a subject steps or walks on the sensor, a change in the total charge of the sensors occurs causing the generated voltage level to increase and decrease depending on the step in or out of the sensor. The shape of the waveform, the amount of charge accumulated or discharged during this process is of interest. In previous work; fall detection and gait estimation, we were interested on whether the sensor is active or not. However, we believe there may be more information in the scavenged signal. We investigated some of the characteristics of the sensor's 
accumulated charge or voltage level with the hope to answer the question: What other information can we get from the walk?

To achieve this goal, we developed a new sensor segment 8 feet long and 18 "wide. As listed in Table II, four different people in terms of gender, weight, height and age walked on the sensor segment four times each maintaining same waking speed and pace. The generated signal is recorded and saved using MSO4034 Tektronix Oscilloscope for further analysis. We applied proper signal processing techniques; computing the signal's spectral density (Fourier transform), total power, the width and duty cycles. Subjects performed walking trials in different settings: Bare foot, and with shoes. Also, the sensors were covered by threaded carpet, or vinyl material. All walks for given setups were performed at the same time to reduce the effect of the weather conditions: temperate and relative humidity. These environmental conditions have effect on static charge build.

TABLE II SUBJECTS’ AGE, WEIGHT, AND HEIGHT

\begin{tabular}{|c|c|c|r|}
\hline Subject & Weight & Height & \multicolumn{1}{|c|}{ Age } \\
\hline Male - Adult & $2001 \mathrm{~b}, 90.72 \mathrm{Kg}$ & $5^{\prime} 9^{\prime \prime}, 174 \mathrm{~cm}$ & 40 \\
\hline Female- Adult & $150 \mathrm{lb}, 68 \mathrm{Kg}$ & $5^{\prime} 3^{\prime \prime}, 160 \mathrm{~cm}$ & 31 \\
\hline Female- Child & $98.41 \mathrm{~b}, 44.63 \mathrm{Kg}$ & $4^{\prime} 8^{\prime \prime}, 142 \mathrm{~cm}$ & 12 \\
\hline Male - Child & $49.71 \mathrm{~b}, 22.5 \mathrm{Kg}$ & $3^{\prime} 11^{\prime \prime}, 119 \mathrm{~cm}$ & 8 \\
\hline
\end{tabular}

\section{2 $\backslash$ 3.Experimental results}

Here we show the results of the improved fall detection algorithms. Additionally, we show how well the smart carpet estimated gait matches the GAITRite, which is used 
as gold standard for gait measurements. Finally, we show the waveform generated by the activating the sensor, and it's charging profiles.

\section{a. Fall Detection Algorithms}

We measured the performance of the fall detection algorithms by counting the number of fall patterns that were detected as falls and we provided both the sensitivity, and specificity of the detected falls on all patterns done by 10 volunteers. Table III shows the sensitivity and specificity for the two methods and their combinations. It also shows the configurations; windows size (WS), and threshold (TH) used for the decision. The best results of $95 \%$ sensitivity and $85 \%$ specificity were achieved using $\mathrm{WS}=7, \alpha=0.3$, and 1.2 for Convex Hull and Heuristic methods respectively. Clearly combining the two methods increased the true negatives (TN).

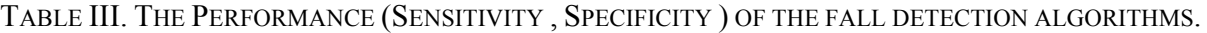

\begin{tabular}{|l|c|c|}
\hline \multicolumn{1}{|c|}{ Algorithm } & $\begin{array}{c}\text { Fall Detected as } \\
\text { Fall (Sensitivity) }\end{array}$ & $\begin{array}{c}\text { No Fall detected as No } \\
\text { Fall (Specificity) }\end{array}$ \\
\hline Convex Hull Area & $90 \%$ & $80 \%$ \\
\hline Heuristic & $86 \%$ & $80 \%$ \\
\hline $\begin{array}{l}\text { Convex Hull AND Heuristic } \\
\text { (Inclusive) }\end{array}$ & $75 \%$ & $89 \%$ \\
\hline $\begin{array}{l}\text { Convex Hull OR Heuristic } \\
\text { (Exclusive) }\end{array}$ & $95 \%$ & $85 \%$ \\
\hline \multicolumn{1}{|c|}{ Threshold criteria: $\boldsymbol{W S = 7 ,} \boldsymbol{\alpha}_{\text {Hull }}=\mathbf{0 . 3 ,} \boldsymbol{\alpha}_{\text {Heuristic }}=\mathbf{1 . 2}$} \\
\hline
\end{tabular}

\section{b. Gait Estimation System Performance}

The t-test shows how well the smart carpet gait estimating system matches the GAITRite system. Table IV shows the statistical t-test2 for the smart carpet and the 
GAITRite systems. The p-value for walking speed is 0.55 , which means that the two systems are not statistically different. However, for Stride time $\mathrm{p}=0.0017$, which means

TABLE IV TTEST2 - STATISTICS FOR WALKING SPEED, STRIDE TIME, AND STRIDE LENGTH

\begin{tabular}{|l|l|l|l|l|l|}
\hline Variable & Mean & Stdev & T-Test & $\mathbf{P}$ & $\mathbf{H}_{\mathbf{0}}$ \\
\hline Walking Speed & 42.10 & 6.8375 & -0.5934 & 0.5538 & 0 \\
\hline Stride Time & 1.30 & 0.1354 & 3.1929 & 0.0017 & 1 \\
\hline Stride Length & 54.91 & 6.3838 & 2.1962 & 0.0297 & 1 \\
\hline
\end{tabular}

that the differences are statistically significant. This is due to the method we measured the stride time and length; time of first heel stride, or full foot on carpet. We believe a higher resolution sensors will enable us to obtain better correlation and significance measures. Similarly using the Mann-Whitney U test; the p-value is 0.45326 for walking speed, which means that it is not significant at $\mathrm{p}<0.05$. And it is significant for the other two parameters.

In the scatter plot Figure 5 shows visual of the differences between the two systems. The best fitting line (trend line) with the correlation equations are shown for three gait parameters; walking speed, stride time and stride length. The R-squared of $93 \%$ for walking speed shows great agreement between the two systems. Additionally, our calculations showed strong relation between the two systems. As expected, the r-squared from Stride time is low meaning more variability between the two systems. 


\section{Walking Speed}

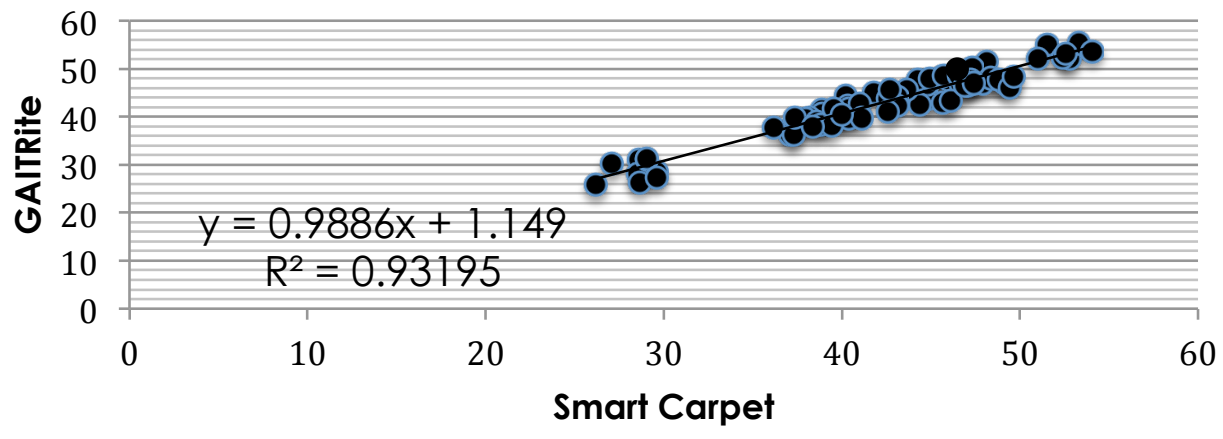

Figure 5-a Scatter plot with fitting line and variation factor for both systems with respect to walking speed.

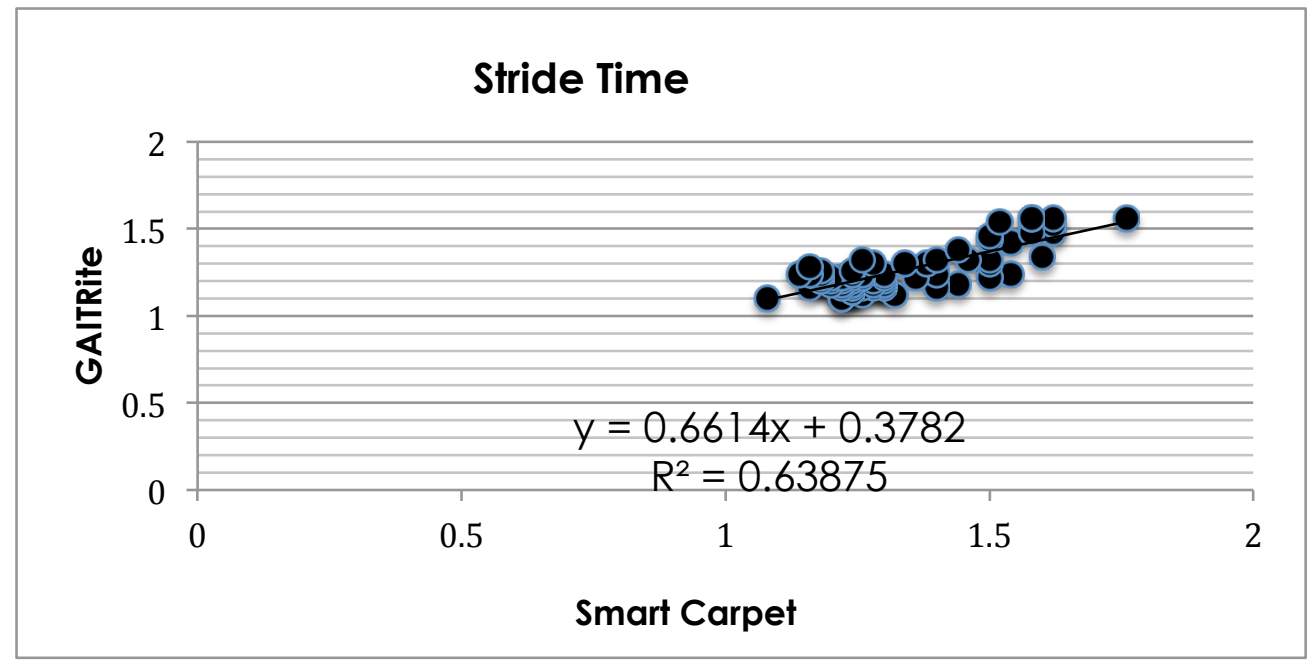

Figure 5-b Scatter plot with fitting line and variation factor for both systems with respect to stride time.

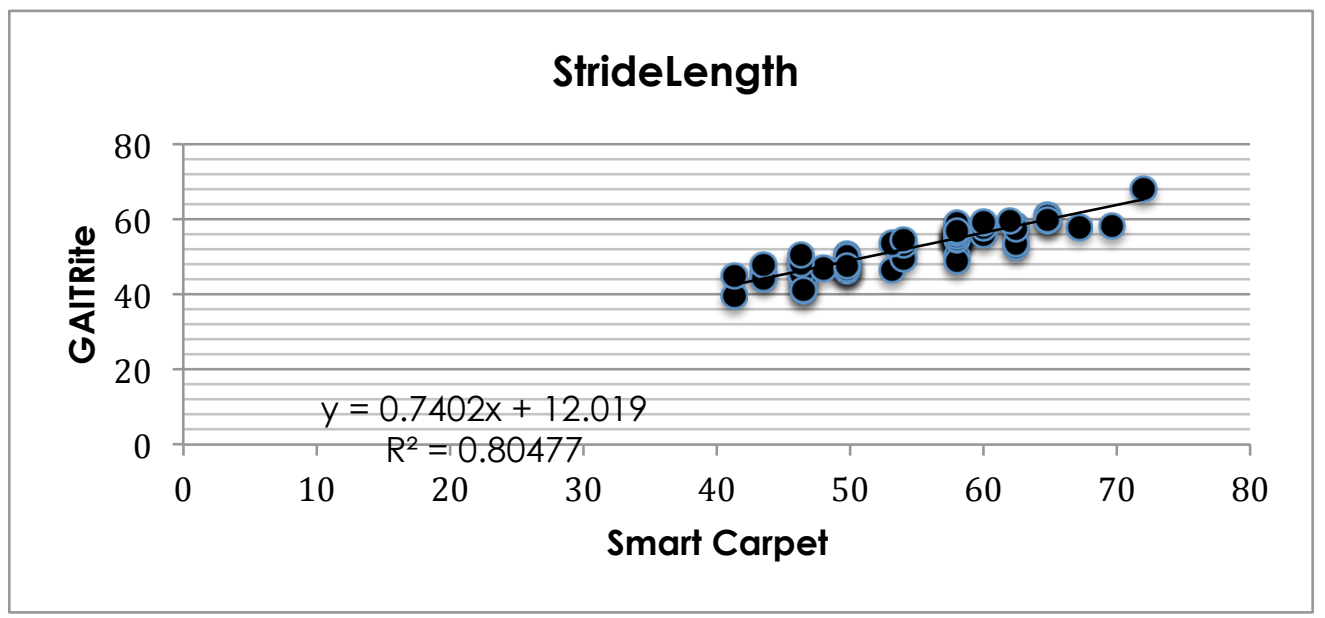

Figure 5-c Scatter plot with fitting line and variation factor for both systems with respect to stride lenoth. 


\section{b. Scavenged Charge Characteristics}

Subjects performed the walks on different sensors surfaces; threaded carpet and transparent vinyl. Results showed sensors covered with different material have correspondingly different signal behavior. The measured power (amount of charge accumulated) differs with different material used. Table $\mathrm{V}$ shows the average power measured for all four subjects' trials on Vinyl and threaded cloth type carpet. Figure 7 shows sample of the scavenged signals from the sensors on both surfaces for a $2001 \mathrm{~b}$ male, walking on the same 8-foot segment covered by both Vinyl and threaded carpet. This was repeated for 4 subjects, and each subject generated different waveforms. We have traced the footfalls and observed that as the subjects took steps there was charge build up or discharge. The overall droop or in the signal indicated a builddown of total

TABLE V AVERAGE POWER MEASURED USING VINYL, AND CARPET SURFACES

\begin{tabular}{|c|c|c|}
\hline Subject/Voltage (V) & $\begin{array}{c}\text { Vinyl } \\
\text { (Power Unit) }\end{array}$ & $\begin{array}{c}\text { Threaded Carpet (Power } \\
\text { Unit) }\end{array}$ \\
\hline Male - Adult & 2543 & 1620 \\
\hline Female- Adult & 951 & 671 \\
\hline Female- Child & 929 & 162 \\
\hline Male - Child & 411 & 229 \\
\hline
\end{tabular}

charge as the steps progressed.

However, we need to develop criteria and measurements to understand and interpret the scavenged signal, since different people have different waveforms, and charge build-up characteristics. It seems interesting to investigate considering other factors like weather, shoe type, sensor material...etc. 

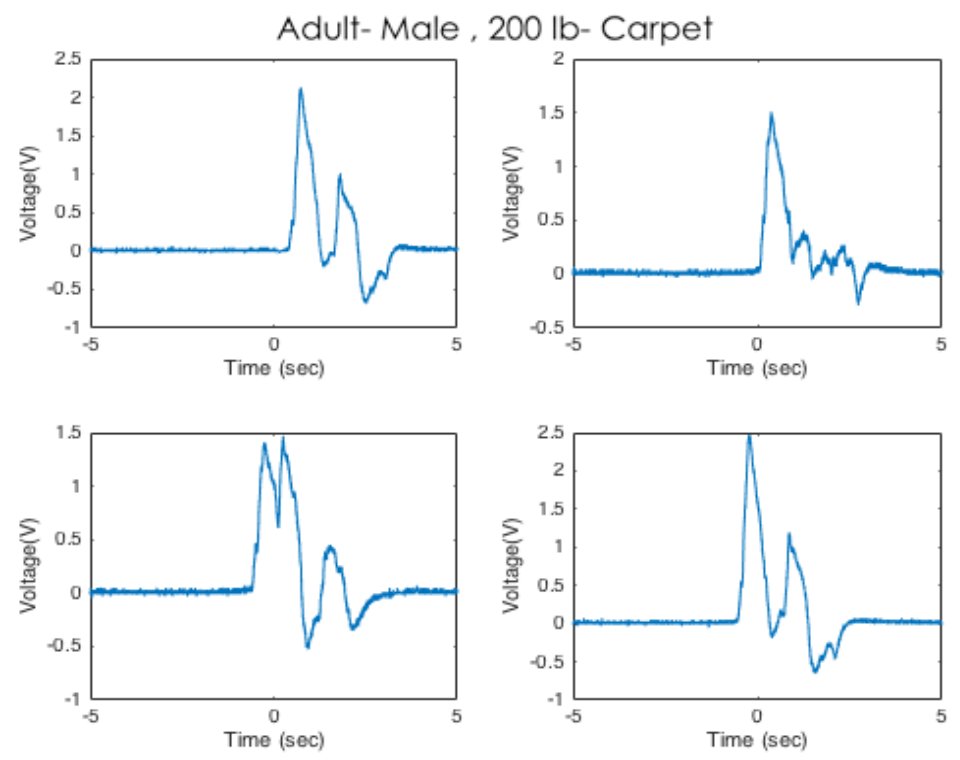

(a)

Adult- Male , 200 lb- Vinyl
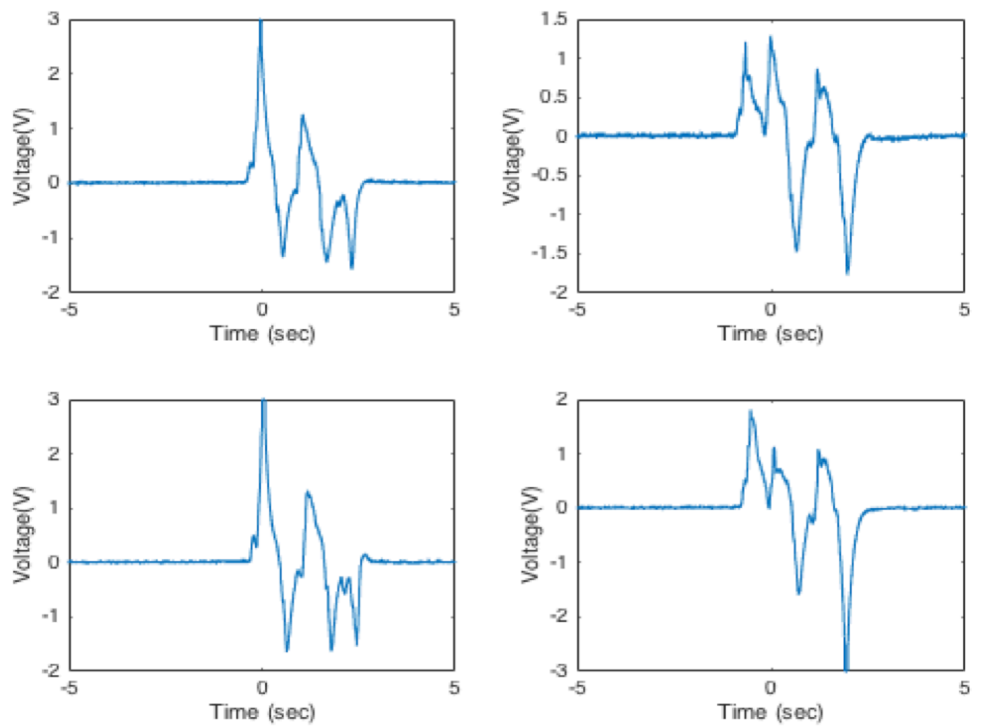

(b)

Figure 6 Scavenged signals for $200 \mathrm{lb}$. adult male walking on sensors covered by carpet (a) Vinyl (b) 


\subsection{Discussion and Conclusion}

The two algorithms; convex hull area, and the heuristic active sensors' count enhanced fall detection for the floor based personnel detection system. Results showed improvements in both the sensitivity and specificity. Most signal scavenging systems, or any signals-based systems are noise prone by nature. Fusing more than one method will help to minimize the effect of the noise on the system performance.

Our system was used to extract and estimate the gait parameters and compare them to the gold standards through the use of GAITRite. Results showed a very acceptable match between the two systems, with $90 \%$ correlation, and no statistically difference for walking speed and acceptable margin for stride length. These results are very helpful since of importance is the relative change in gait parameters. In general aging slows a person down and we will be able to detect this slow-down as a change in gait while we keep recording sensor activity $24 / 7$ over a period of years.

Both fall detection and Gait estimation are based on the measured voltage generated by the sensor and whether that voltage exceeded certain threshold or not to consider the sensor active.

\section{References}


[1] H. W. Tyrer, M. A. Aud, G. Alexander, M. Skubic and M. Rantz, "Early Detection of Health Changes In Older Adults," 2007 29th Annual International Conference of the IEEE Engineering in Medicine and Biology Society, Lyon, 2007, pp.4045-4048. doi: 10.1109/IEMBS.2007.4353221

[2] G. K. Vincent, V. A. Velkoff, The Next Four Decades: The Older Population in the United States: 2010 to 2050, US Department of Commerce, Economics and Statistics Administration, U.S. Census Bureau, 2010

[3] D. Hodgins, "The Importance of Measuring Human Gait," Medical Device Technology. 2008 Sep; 19(5):42, 44-7.

[4] World Health Organization: Global report on falls prevention in older age.[http://www.who.int/ageing/publications/Falls_preventio 7March.pdf]

[5] D. Jiangpeng, B. Xiaole, Y. Zhimin, S. Zhaohui, and X. Dong, "PerFallD: A pervasive fall detection system using mobile phones," in Pervasive Computing and Communications Workshops (PERCOM Workshops), 2010 8th IEEE International Conference on, 2010, pp. 292-297

[6] C. G. Moran, R.T. Wenn, M. Sikand, A.M. Taylor, Early mortality after hip fracture: is delay before surgery important", J. of Bone and Joint Surgery, pp. 483-9, 2005.

[7] Estimating Walking Speed, Stride Length, and Stride Time using a Passive Floor Based Electronic Scavenging System. 2017 IEEE Sensors Applications Symposium (SAS)-2017 IEEE Sensors Applications Symposium. Accepted, to appear March 2017.

[8] Viccaro LJ, Perera S, Studenski SA. Is timed up and go better than gait speed in predicting health, function, and falls in older adults? J Am Geriatr Soc. 2011; 59(5):887 92.

[9] Peel NM, Kuys SS, Klein K. Gait speed as a measure in geriatric assessment in clinical settings: a systematic review. J Gerontol Ser A Biol Sci Med Sci. 2013; 68(1):39-46.

[10] Van Kan GA, Rolland Y, Andrieu S, Bauer J, Beauchet O, Bonnefoy M, Cesari M, Donini L, Gillette-Guyonnet S, Inzitari $\mathrm{M}$, et al. Gait speed at usual pace as a predictor of adverse outcomes in community-dwelling older people an international academy on nutrition and aging (iana) task force. J Nutr Health Aging. 2009; 13(10):881-9.

[11] Rochat S, Büla CJ, Martin E, Seematter-Bagnoud L, Karmaniola A, Aminian K, Piot-Ziegler C, Santos-Eggimann B. What is the relationship between fear of falling and gait in well-functioning older persons aged 65 to 70 years? Arch Phys Med Rehabil. 2010; 91(6):879-84.

[12] Rochat S, Büla CJ, Martin E, Seematter-Bagnoud L, Karmaniola A, Aminian K, Piot-Ziegler C, Santos-Eggimann B. What is the relationship between fear of falling and gait in well-functioning older persons aged 65 to 70 years? Arch Phys Med Rehabil. 2010; 91(6):879-84.

[13] Taylor ME, Ketels MM, Delbaere K, Lord SR, Mikolaizak AS, Close JC. Gait impairment and falls in cognitively impaired older adults: an explanatory model of sensorimotor and neuropsychological mediators. Age Ageing. 2012; 41(5):665-9.

[14] Stone EE, Skubic M. Capturing habitual, in-home gait parameter trends using an inexpensive depth camera. In: Engineering in Medicine and Biology Society (EMBC), 2012 Annual International Conference of the IEEE. IEEE; 2012. p. 5106-9. doi:10.1109/EMBC.2012.6347142.

[15] E. E. Stone and M. Skubic, "Unobtrusive, Continuous, In-Home Gait Measurement Using the Microsoft Kinect," in IEEE Transactions on Biomedical Engineering, vol. 60, no. 10, pp. 2925-2932, Oct. 2013. doi: 10.1109/TBME.2013.2266341

[16] Alwan, M.; Rajendran, P.J.; Kell, S.; Mack, D.; Dalal, S.; Wolfe, M.; Felder, R. A smart and passive floor-vibration based fall detector for elderly. In Proceedings of the Second Information and Communication Technologies, Damascus, Syria, 24-28 April 2006; pp. 1003-1007

[17] Liu, Liang, et al. "Fall detection using Doppler radar and classifier fusion. "Biomedical and Health Informatics (BHI), 2012 IEEE-EMBS International Conference on. IEEE, 2012. 
[18] Li, Y., K. C. Ho, and M. Popescu. "A Microphone Array System for Automatic Fall Detection." IEEE Trans Biomed Eng 59 (2012): 1291 - 301.

[19] M. Popescu, Y. Li, M. Skubic, and M. Rantz, "An acoustic fall detector system that uses sound height information to reduce the false alarm rate," in Engineering 62

[20] R. Orr and G. Abowd, "The Smart Floor: A Mechanism for Natural User identification and tracking," Proceedings of Conference on Human Factors in Computing Systems, CHI'00 extended abstracts on human factors in computing systems, ACM, NY,2000, Pg. 275-276.

[21] Alwan, M.; Rajendran, P.J.; Kell, S.; Mack, D.; Dalal, S.; Wolfe, M.; Felder, R. A smart and passive floor-vibration based fall detector for elderly. In Proceedings of the Second Information and Communication Technologies, Damascus, Syria, 24-28 April 2006; pp. 1003-1007.

[22] Neelgund R., "Floor sensor development using signal scavenging for personnel detection system", University of Missouri, Columbia 2010. Master's thesis for fulfillment of MS Degree.

[23] Chinchao Suriyakul," a new circuit for personnel detection using signal scavenging", University of Missouri, Columbia 2012. Master's thesis for fulfillment of MS Degree.

[24] Gadre K., "Fall detection system using low cost computing and online Communication", University of Missouri, Columbia 2012. Master's thesis for fulfillment of MS Degree.

[25] F. Muheidat and H. W. Tyrer, "Can we make a carpet smart enough to detect falls?," 2016 38th Annual International Conference of the IEEE Engineering in Medicine and Biology Society (EMBC), Orlando, FL, USA, 2016, pp. 5356-5359. doi: 10.1109/EMBC.2016.7591937

[26] M. Rantz, M. Aud, G. Alexander, B. Wakefield, M. Skubic, R. H. Luke, D. Anderson, and J. Keller, "Falls, technology, and stunt actors: New approaches to fall detection and fall risk assessment," J. Nursing Care Quality, vol. 23, no. 3, pp. 195-201, 2008

[27] Barber, C. Bradford, David P. Dobkin, and Hannu Huhdanpaa. "The quick hull algorithm for convex hulls." ACM Transactions on Mathematical Software (TOMS) 22.4 (1996): 469-483

[28] Shoelace algorithm https://en.wikipedia.org/wiki/Shoelace_formula

[29] https://en.wikipedia.org/wiki/Student\%27s_t-test

[30] JH Klotz, A computational Approach to Statistics, 2006

[31] https://en.wikipedia.org/wiki/relation and dependence

\subsection{Closing}

We had additional material that is relevant to this chapter covered in chapter 3 and initial work related to chapter 4 . In addition, we evaluated two fall detection algorithms on the data collected from our previous work [3].This paper was submitted before we had new insight into feature extraction methodology to study the scavenged signal characteristics. 


\section{DERIVING INFORMATION FROM LOW SPATIAL RESOLUTION FLOOR-BASED PERSONNEL}

\section{DETECTION SYSTEM}

\subsection{Introduction}

We have developed preliminary work, which we describe in a paper titled" Deriving Information from Low Spatial Resolution Floor-Based Personnel Detection System"[37].

It provides the count of multiple people walking on the smart carpet. The paper was submitted to CHASE'17- The Second IEEE/ACM Conference on Connected Health: Applications, Systems, and Engineering Technologies. We submitted this paper as a 2page poster paper, which was the only option available. It summarizes the initial work we did on developing algorithms to reliably determine the count of unknown number of people walking on the carpet. In addition, the paper provides a summary of gait estimation measurements, and smart carpet system performance compared to the GAITRite mat. We also evaluated fall detection algorithms on an existing dataset. 


\subsection{Manuscript: Deriving Information from Low Spatial Resolution Floor-Based Personnel Detection System \\ Fadi Muheidat and Harry W. Tyrer*, PhD \\ Electrical Engineering and Computer Science Department, University of Missouri-Columbia}

Abstract-We developed a low cost floor-based personnel detection system, we call a smart carpet, which consists of a sensor pad placed under a carpet; the electronics reads walking activity to provide an automated health monitoring and alert system. We extended the functionalities of the smart carpet to improve its ability to detect falls, alert health care personnel, estimate gait parameters, and count number of people walking on the carpet. Results showed that our system detects falls, using computational intelligence techniques, with $96.2 \%$ accuracy and $81 \%$ sensitivity and $97.8 \%$ specificity. We extracted and estimated gait parameters, and compared it to the GAITRite system (the gold standard), so that the system reliably estimates the walking speed, stride length and stored time with percentage errors of $1.43 \%,-4.32 \%$, and $-5.73 \%$ respectively. Recent results show that the system can count number of people with high accuracy. We developed a database system and web applications for immediate alerts on adverse events, and retain these data in the cloud for years, if necessary, to provide further data analysis.

* Corresponding Author: Harry W, Tyrer, Professor Emeritus, is with University of Missouri, Columbia, MO 65211, USA (Tel. +1- 573-882 6489; Fax: +1-573-882-0397; e-mail: tyrerh@ missouri.edu). 


\section{2\} 1 \text { Background }}

Research focused on older adults continues to promote successful aging, enhance the overall quality of life, provide adequate medical care, and to keep health care cost under control. A major advancement is linking their sensors data into connected health system [1]. People with dementia have limited ability to maintain and use wearable devices, giving rise to a passive floor-based personnel-monitoring system, which may be a solution [2]. We have enhanced the system to detect falls, count multiple people on the carpet, and estimate gait parameters. Recent research shows that changes in gait

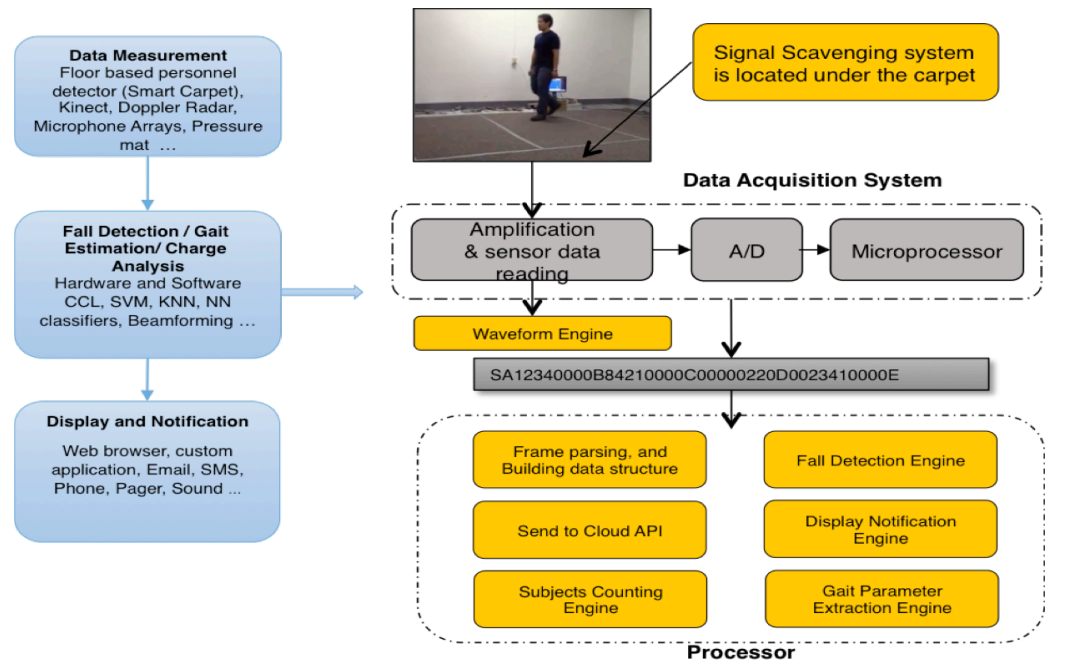

Figure. 1 System overview: context-aware system consists of Data acquisition system,

parameters may be predictive of future falls and adverse events in older adults - hence fall risk. Falls may presage physical functional decline [3] and fall risks [4]. We describe a system that is unobtrusive and continuously monitors the daily activities of elderly people. The early detection of gait decline will benefit the senior by providing an assessment of the risk of falls. 


\section{2\2 System Overview and Experiments}

The system uses signal-scavenging technique to detect presence of the person. Signal scavenging uses a sensor made from a conductive material to pick up stray $60 \mathrm{~Hz}$ noise, which may come from electrical power lines, nearby electrical equipment and other stray electromagnetic signals. Although this strong $60 \mathrm{~Hz}$ signal seems to be useless energy, we have shown it to be a valuable signal source to detect the presence of humans. Our system, as shown in Figure 1 consists of; the Smart Carpet, a floor based personnel detector. Electronics provide the data acquisition system, and processor. Sensor signals converted into digital values with a constant threshold using 10-bit Analog to Digital convertor, and then read by a computer result in an ASCII frame. The software components process the data frames, and use different computational intelligence methods to perform the required operations like fall detection, gait estimation, people counting, data visualization and notification. The smart carpet data acquisition system scans the sensors at $\mathrm{N}$ frames/second. We parse the frame data into binary image that corresponds to the carpet layout. Where ' 1 ' means the sensor is activated, and ' 0 ' means it is not activated. This image becomes the data structure to perform computations. To add smarts to our system, we used different classifiers; Naive Bays, Multilayer Perceptron, Support Vector Machine, and others. We used different computational algorithms to extract features to build a reliable classifier such Connected Component Labeling, Convex Hull, and Heuristics. We used the above-mentioned tools for different experimental setups for fall recognition, gait estimation, and multiple people count. 


\section{A. Fall Detection}

We recruited 10 volunteers; each to performed eight walk-fall patterns. The falling patterns were adapted from previous work by our eldercare technology research group [5]. To validate the accuracy of the fall detection algorithms we recorded the walks in video.

\section{B. Gait Estimation}

We placed a two-segment carpet at the top of the GAITRite [6] mat aligned to the active region of the GAITRite mat, and the subjects walked across the carpet. The walk data are recorded and later used to extract the gait parameters. In total, there are 75 walk sequences. We determined the footfalls by grouping the scanned frames in a window of size WN, and then counted the number of active sensors. We set a threshold (TH), and sensors count greater than that value we considered a valid footfall. We measured the walking distance, and walking time, and then computed the walking speed, stride time and stride length. We calculated strength of linear correlation, and fluctuations of one of the system with respect to the other by computing the correlation coefficients, and the coefficient of determination $\left(r^{2}\right)$ respectively for Walking Speed, Stride Time and Stride length.

\section{People count}

Four people performed 10 walking sequences for different scenarios: individual, two, three and four persons walking in the same and opposite directions. We calculated the average number of sensors activated by each walk. We then divided by the mean of active sensors produced by individual walks for the persons who performed the walk. We 
rounded the result to obtain the count of people. This allows us to determine the count of unknown people walking on the carpet.

\section{$7.2 \backslash 3$ Results}

Results showed that Computational Intelligence techniques detect falls with $96.2 \%$ accuracy and $80 \%$ sensitivity and $97.8 \%$ specificity [7]. Our system was able to estimate the gait parameters with high correlation between the carpet and the GAITRite. Table I shows the average activated sensors count produced by each walk. The

TABLE I AVERAGE ACTIVE SENSORS COUNT, AND COUNT OF PEOPLE WALKING ON THE CARPET

\begin{tabular}{|l|c|c|c|}
\hline Scenario & $\begin{array}{c}\text { Average active } \\
\text { sensors count }\end{array}$ & $\begin{array}{c}\text { Average active sensors count for } \\
\text { persons who performed the walk }\end{array}$ & $\begin{array}{c}\text { People } \\
\text { Count }\end{array}$ \\
\hline One Person & 13.62 & 13.62 & 1 \\
\hline Two persons-Same direction & 27 & 14.20 & 2 \\
\hline $\begin{array}{l}\text { Two persons-Opposite } \\
\text { direction }\end{array}$ & 25 & 14.20 & 3 \\
\hline Three persons -Same & 49 & 14.10 & 4 \\
\hline directions & 58 & 13.62 & 4 \\
\hline Four persons -Same & 48 & & 13.62 \\
\hline direction & & & \\
\hline Four persons - Opposite \\
direction
\end{tabular}

fourth column shows the number if people detected by dividing the second column by the third column and round the results to nearest integer. Results show 100\% accuracy. Table 
II shows the percentage difference mean and standard deviation of walking speed, stride time, and stride length for each of the walking sequences as computed for both systems. The $\boldsymbol{r}^{2}$ of $93 \%$ for walking speed shows great agreement between the two systems. In addition to fall detection and gait estimation we developed a database system and web applications to retain these data for years. We can display this data in real-time and for all activities in the carpet for extensive data analysis any time in the future.

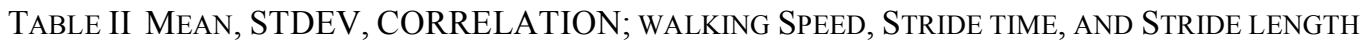

\begin{tabular}{|l|l|l|l|}
\hline Variable & $\begin{array}{l}\text { Mean } \\
\text { difference }\end{array}$ & Stdev & Correlation (R2) \\
\hline Walking Speed & 1.43 & 4.39 & 0.932 \\
\hline Stride Time & -5.73 & 7.19 & 0.805 \\
\hline Stride Length & -4.32 & 6.04 & 0.639 \\
\hline
\end{tabular}

\section{2\} 4 \text { Discussion }}

Falls for an individual are a rare but high impact event, and affect a large fraction of the population. So it is important to monitor, but not act until necessary. Further the system generates data $24 / 7$ and it can be recorded and processed to emphasize changes in activity as obtained by gait. Finally since a plurality of people walking on the floor can mimic a fall event, it is important that the system distinguish between a plurality of people and a fall event. 


\section{References}

[1] Popescu M \& Florea E, "Linking Clinical Events in Elderly to In-home Monitoring Sensor Data: A Brief Review and a Pilot Study on Predicting Pulse Pressure," Journal of Computing Science and Engineering, March 2008, 2(1):180-199

[2] Neelgund R.,"Floor sensor development using signal scavenging for personnel detection system", University of Missouri, Columbia 2010. Masters thesis for MS Degree.

[3] Peel NM, Kuys SS, Klein K. Gait speed as a measure in geriatric assessment in clinical settings: a systematic review. J Gerontol Ser A Biol Sci Med Sci. 2013;68(1):39-46.

[4] Taylor ME, Ketels MM, Delbaere K, Lord SR, Mikolaizak AS, Close JC. Gait impairment and falls in cognitively impaired older adults: an explanatory model of sensorimotor and neuropsychological mediators. Age Ageing. 2012;41(5):665-9.

[5] M. Rantz, M. Aud, G. Alexander, B. Wakefield, M. Skubic, R. H. Luke, D. Anderson, and J. Keller, "Falls, technology, and stunt actors: New approaches to fall detection and fall risk assessment," J. Nursing Care Quality, vol. 23, no. 3, pp. 195-201, 2008

[6] Peel NM, Kuys SS, Klein K. Gait speed as a measure in geriatric assessment in clinical settings: a systematic review. J Gerontol Ser A Biol Sci Med Sci. 2013;68(1):39-46.

[7] F. Muheidat and H. W. Tyrer, "Can we make a carpet smart enough to detect falls?," 2016 38th Annual International Conference of the IEEE Engineering in Medicine and Biology Society (EMBC), Orlando, FL, 2016, pp. 5356 5359. doi: 10.1109/EMBC.2016.7591937

\subsection{Closing}

We had additional material that is relevant to this short paper covered in chapters 3 , and 5. This was submitted as a two - page poster paper. By the time we found about it, the time already passed for the full paper submission. Because this is a biomedical engineering conference, we wanted to provide more of a sum 


\section{CHAPTER 6: DISCUSSION AND CONCLUSION}

\subsection{Summary}

The work in this dissertation demonstrated possible clinically relevant applications for the smart carpet. Not only it can detect falls, but also it can estimate gait, and assess the plurality of people. Gait assessment will have clinical uses; by storing the data in the cloud, we can provide periodic updates to the residents, which could have beneficial diagnostic information. We demonstrated gait estimation in two papers one published in IEEE Transactions in Sensors Applications Symposium (SAS) in 2017, and described in chapter 3, and the other is submitted to the 18th International Conference on Internet Computing and Internet of Things, ICOMP'17, and described in chapter 6 . We found that we can detect the plurality of people. We produced a paper and submitted to the 3rd International Conference on Health Informatics and Medical Systems, HIMS'17, and described in chapter 5. We spent a fair amount of time trying to get additional information from the waveform generated from the sensors. With this waveform information, we required formal computation intelligence techniques to extract features to understand the characteristics of the voltage signal generated by people walking on the smart carpet. However, we found a lot of variability in the system, and more work needs to be done to study this waveform behavior. Overall, the results obtained are satisfactory and encouraging to the development of standalone application to be installed in elderly residents or nursing homes. 


\subsection{Contributions}

The contributions of this dissertation involve carpet design and construction, electronics design, experimental setup, data collection, processing, and analysis. The followings are our formal produced work that some has been published and others submitted.

\subsubsection{Gait Estimation:}

Our system was used to extract and estimate the gait parameters and achieved an excellent agreement compared to the gold standards through the use of GAITRite. Results showed a very acceptable match between the two systems, with $90 \%$ correlation, and no statistically difference for walking speed and acceptable margin for stride length. These results are very helpful since of importance is the relative change in gait parameters. In general aging slows a person down and we will be able to detect this slow-down as a change in gait while we keep recording sensor activity $24 / 7$ over a period of years. We compared our system with other systems developed by the Eldercare Technology research group: Kinect, and web cameras. These data had a high level of agreement.

\subsubsection{Waveform Study}

We investigated some of the characteristics of the sensor's accumulated charge or voltage level with the hope to answer the question: What other information can we get from the walk? We performed a set of experiments on different configurations, number and characteristics of participants. The sensors' voltage waveforms behaved differently corresponding to different people and set of walking trials. By inspection, we could not 
distinguish different people due to the high variability like the covering material, shoe type, and the environmental conditions, which affect the behavior of the scavenged signal. However, we were able to use computational features, by extracting the Mel Frequency Cepstral Coefficients (MFCC), and using a formal computation intelligence to distinguish different people with an average accuracy of $82 \%$, given that the experiments were performed within the same day.

\subsubsection{Count plurality of people}

We extended the functionality of the smart carpet to count the number of people walking on the carpet. We used the total count of activated sensors compared to the average of individual walks sensors count, and were able to count the number of people with close to $100 \%$ accuracy. Additionally, we developed hybrid model that uses the count of activated sensors, and the number and size of the subgroups formed by the activated sensors. We were able to count the number of people at any given time with accuracy up to $100 \%$. The number of people walking, the spatial distance that separates the people, and the ratio of the number of people walking to the actual carpet size influenced the results.

\subsubsection{Fall detection}

Most signal scavenging systems, or any signal-based systems are noise prone by nature. Fusing more than one method will help to minimize the effect of the noise on the system performance. In our previous work [3] we used the smart carpet to detect fall. Here we proposed two algorithms to enhance the fall detection accuracy. The two algorithms we propose are convex hull area, and a heuristic based off the active sensors' 
count in certain activated sensor layout. Results showed improvements in both sensitivity (95\%) and Specificity (85\%) when combining both methods exclusively.

\subsection{Future work}

The smart carpet system was able to measure and extract gait parameters. Enhancing the footfall detection, and measuring accurately travelled distance will yield better matching and reduced percentage difference compared to GAITRite and Vicon motion capture system. Both fall detection and Gait estimation are based on the measured voltage generated by the sensor and whether that voltage exceeded certain threshold or not, so

further waveform studies could help extract more discriminatory features that assist in achieving more accurate parameter extractions, and people profiling. 


\section{BIBLIOGRAPHY}

[1] Muheidat, F, "Adding Intelligence to a Floor based Array Personnel Detector", University of Missouri, Columbia 2015. Master's thesis for fulfillment of MS Degree.

[2] Neelgund R., "Floor sensor development using signal scavenging for personnel detection system", University of Missouri, Columbia 2010. Master's thesis for fulfillment of MS Degree.[24]

[3] F. Muheidat and H. W. Tyrer, "Can we make a carpet smart enough to detect falls?"2016 38th Annual International Conference of the IEEE Engineering in Medicine and Biology Society (EMBC), Orlando, FL,2016, pp.5356-5359. doi: 10.1109/EMBC.2016.7591937 [25]

[4] F. Muheidat, H. W. Tyrer, M. Popescu and M. Rantz, "Estimating walking speed, stride length, and stride time using a passive floor based electronic scavenging system,"2017 IEEE Sensors Applications

Symposium (SAS), Glassboro, NJ, USA, 2017, pp. doi: $10.1109 /$ SAS.2017.7894112

[5] F. Muheidat, H. W. Tyrer. "Floor Based Sensor System: Additional Intelligence, Gait Estimation, and Scavenging Charging Characteristics". Accepted to ICOMP'17- The 18th International Conference on Internet Computing and Internet of Things. Las Vegas, Nevada, USA, 2017.

[6] F. Muheidat, H. W. Tyrer. "Counting Multiple People on a Floor Based Array sensor system". Accepted to HIMS'17 - The 3rd International Conference on Health Informatics and Medical Systems. Las Vegas, Nevada, USA, 2017.

[7] H. W. Tyrer, M. A. Aud, G. Alexander, M. Skubic and M. Rantz, "Early Detection of Health Changes in Older Adults," 2007 29th Annual International Conference of the IEEE Engineering in Medicine and Biology Society, Lyon, 2007, pp.4045-4048. doi: 10.1109/IEMBS.2007.4353221

[8] D. Jiangpeng, B. Xiaole, Y. Zhimin, S. Zhaohui, and X. Dong, "PerFallD: A pervasive fall detection system using mobile phones," in Pervasive Computing and Communications Workshops (PERCOM Workshops), 2010 8th IEEE International Conference on, 2010, pp. 292-297

[9] Viccaro LJ, Perera S, Studenski SA. Is timed up and go better than gait speed in predicting health, function, and falls in older adults? J Am Geriatr Soc. 2011;59(5):887 92.

[10] Peel NM, Kuys SS, Klein K. Gait speed as a measure in geriatric assessment in clinical settings: a systematic review. J Gerontol Ser A Biol Sci Med Sci. 2013;68(1):39-46.

[11] Van Kan GA, Rolland Y, Andrieu S, Bauer J, Beauchet O, Bonnefoy M, Cesari M, Donini L, Gillette-Guyonnet S, Inzitari $\mathrm{M}$, et al. Gait speed at usual pace as a predictor of adverse outcomes in community-dwelling older people an international academy on nutrition and aging (iana) task force. J Nutr Health Aging. 2009;13(10):881-9.

[12] Rochat S, Büla CJ, Martin E, Seematter-Bagnoud L, Karmaniola A, Aminian K, Piot-Ziegler C, Santos-Eggimann B. What is the relationship between fear of falling and gait in well-functioning older persons aged 65 to 70 years? Arch Phys Med Rehabil. 2010;91(6):879-84.

[13] Taylor ME, Ketels MM, Delbaere K, Lord SR, Mikolaizak AS, Close JC. Gait impairment and falls in cognitively impaired older adults: an explanatory model of sensorimotor and neuropsychological mediators. Age Ageing. 2012;41(5):665-9. 
[14] Stone EE, Skubic M. Capturing habitual, in-home gait parameter trends using an inexpensive depth camera. In: Engineering in Medicine and Biology Society (EMBC), 2012 Annual International Conference of the IEEE. IEEE; 2012. p. 5106-9. doi:10.1109/EMBC.2012.6347142.

[15] D. Giansanti, G. Maccioni and V. Macellari, The development and test of a device for the reconstruction of $3 \mathrm{D}$ position and orientation by means of a kinematic sensor assembly with rate gyroscopes and accelerometers, IEEE Trans. Biomed. Eng.

[16] B.R. Greene, A. O’Donovan, R. Romero-Ortuno, L. Cogan, C. N. Scanaill, and R.A. Kenny, "Quantitative Falls Risk Assessment Using the Timed Up and Go Test," IEEE Trans. on Biomedical Engineering, vol. 57, no. 12, Dec., 2010, pp. 2918-2926.

[17] Y. Higashi, et al., "Quantitative evaluation of movement using the timed up-and-go test," IEEE Eng. in Med and Bio Magazine, 27(4):38-46, 2008.

[18] MR. Narayanan, et al., "Longitudinal falls-risk estimation using triaxial accelerometry," IEEE Trans on Biomed Eng., 57(3):534, 2010.

[19] G. Demiris, M. Rantz, M. Aud, K. Marek, H. Tyrer, M. Skubic, and A. Hussam, "Older Adults' Attitudes Towards and Perceptions of 'Smarthome' Technologies: a Pilot Study," Medical Informatics and The Internet in Medicine, June, 2004, vol. 29, no. 2, pp. 87-94.

[20] E. Stone, M. Skubic, M. Rantz, C. Abbott, and S. Miller, "Average In-Home Gait Speed: Investigation of a New Metric for Mobility and Fall Risk Assessment of Elders," submitted, Gait and Posture, 2013.

[21] E. Stone and M. Skubic, "Unobtrusive, Continuous, In-Home Gait Measurement Using the Microsoft Kinect,” IEEE Transactions on Biomedical Engineering, 60(10):2925-32, 2013.

[22] M. Rantz, M. Skubic, C. Abbott, C. Galambos, Y. Pak, D. Ho, E. Stone, L. Rui, J. Back, and S. Miller, "In-home Fall Risk Assessment and Detection Sensor System," Journal of Gerontological Nursing, 39(7):18-22, 2013.

[23] E. Stone and M. Skubic, "Evaluation of an Inexpensive Depth Camera for In-Home Gait Assessment," Journal of Ambient Intelligence and Smart Environments, 3(4):349-361, 2011.

[24] E. Stone and M. Skubic, "Mapping Kinect-based In-Home Gait Speed to TUG Time: A Methodology to Facilitate Clinical Interpretation," ICST 7th International Conference on Pervasive Computing Technologies for Healthcare (Pervasive Health), Venice, Italy, 2013.

[25] E. Stone and M. Skubic, "Passive, In-Home Gait Assessment Using an Inexpensive Depth Camera: Initial Results," ICST 6th International Conference on Pervasive Computing Technologies for Healthcare (Pervasive Health), San Diego, CA, 2012.

[26] E. Stone and M. Skubic, "Passive In-Home Measurement of Stride-to-Stride Gait Variability Comparing Vision and Kinect Sensing," IEEE International Conference of the Engineering in Medicine and Biology Society (EMBC), Boston, MA, 2011.

[27] K. Kurita, "Physical activity estimation method by using wireless portable sensor," 2012 IEEE Sensors, Taipei, 2012, pp. 1-4. doi: 10.1109/ICSENS.2012.6411417

[28] Lars Klack, Christian Möllering, Martina Ziefle, and Thomas Schmitz-Rode. Future Care Floor: A sensitive floor for movement monitoring and fall detection in home environments. Wireless Mobile 
Communication and Healthcare. Social Informatics and Telecommunications Engineering, 1, Volume 55, 2011, Heidelberg, Dordrecht, London: Springer, p. 211-218

[29] Robert J. Orr and Gregory D. Abowd, The Smart Floor: A Mechanism for Natural User Identification and Tracking, Graphics, Visualization and Usability (GVU) Center, Georgia Institute of Technology (2000)

[30] Y. Li, K. C. Ho and M. Popescu, "A Microphone Array System for Automatic Fall Detection," in IEEE Transactions on Biomedical Engineering, vol. 59, no. 5, pp. 1291-1301, May 2012. doi: 10.1109/TBME.2012.2186449

[31] Shikha Gupta, Jafreezal Jaafar, "Feature Extraction Using MFCC," University Technology PETRONAS, CIS Dept., Perak, Malaysia, August 2013

[32] E.Stone, "Unobtrusive, In-Home Gait Measurement, Fall Risk Assessment, and Fall Detection Using Depth Imagery,” Ph.D. dissertation, University of Missouri, Columbia, MO, USA, Dec. 2013

[33] F.Wang, "Motion analysis for in-home gait and balance assessment using inexpensive video sensors," Ph.D. dissertation, University of Missouri, Columbia, MO, USA, Jul. 2011.

[34] Hamming Window filter, https://www.mathworks.com/help/signal/ref/hamming.html, accessed 04/28/2017, 5:18 PM.

[35] Discrete Cosine Transform https://users.cs.cf.ac.uk/Dave.Marshall/Multimedia/node231.html, accessed 04/28/2017, 5:21 PM.

[36] Eibe Frank, Mark A. Hall, and Ian H. Witten (2016). The WEKA Workbench. Online Appendix for "Data Mining: Practical Machine Learning Tools and Techniques", Morgan Kaufmann, Fourth Edition, 2016.

[37] F. Muheidat, H. W. Tyrer. Deriving Information from Low Spatial Resolution Floor-Based Personnel Detection System". Accepted CHASE'17- The Second IEEE/ACM Conference on Connected Health: Applications, Systems and Engineering Tech 


\section{APPENDIX}

Appendix A - List of all publications.

Appendix B List of publications on smart carpet not already in the Thesis 


\section{Appendix A - List of all publications}

[1] F. Muheidat, H. W. Tyrer. Deriving Information from Low Spatial Resolution Floor-Based Personnel Detection System". Accepted CHASE'17- The Second IEEE/ACM Conference on Connected Health: Applications, Systems and Engineering Tech

[2] F. Muheidat, H. W. Tyrer. "Floor Based Sensor System: Additional Intelligence, Gait Estimation, and Scavenging Charging Characteristics". Accepted to ICOMP'17- The 18th International Conference on Internet Computing and Internet of Things. Las Vegas, Nevada, USA, 2017.

[3] F. Muheidat, H. W. Tyrer. "Counting Multiple People on a Floor Based Array sensor system". Accepted to HIMS'17 - The 3rd International Conference on Health Informatics and Medical Systems. Las Vegas, Nevada, USA, 2017.

[4] F. Muheidat, H. W. Tyrer, M. Popescu and M. Rantz, "Estimating walking speed, stride length, and stride time using a passive floor based electronic scavenging system,"2017 IEEE Sensors Applications $\begin{array}{lllll}\text { Symposium } \quad(S A S), \quad \text { Glassboro, } & \text { NJ, } & \text { USA, } & \text { 2017, } & \text { pp. }\end{array}$ doi: $10.1109 /$ SAS.2017.7894112

[5] F. Muheidat and H. W. Tyrer, "Can we make a carpet smart enough to detect falls?," 2016 38th Annual International Conference of the IEEE Engineering in Medicine and Biology Society (EMBC), Orlando, FL, USA, 2016, pp. 5356-5359. doi: 10.1109/EMBC.2016.7591937

[6] H. W. Tyrer, F. Muheidat. Estimating Gait Parameters Using a Floor-Based Array Personnel Detector, Alzheimer's Association International Conference (AAIC) 2017, London, UK (Accepted)

[7] F. Muheidat, H. W. Tyrer. Adding Intelligence to a Floor Based Array Personnel Detector, Poster, Alzheimer's Association International Conference (AAIC) 2016, Toronto, ON, Canada, July, 2016

[8] R. O'Connell, T. Banerjee, F. Muheidat, C. Kirkendall, D. Mueller. Spreading Student-Centered Active Learning Through a Teaching Fellows Program, American Society for Engineering Education (ASEE) Midwest Section Conference, 2014. University of Arkansas-Fort Smith. September 2014.

[9] C. Diaz-Ledezma, A. Hussam, F. Muheidat, and A. Wood. Chapter 8 : Assessment Tools: HealthRelated Quality of Life Measurement and Other Hip-Specific Scores in Hip Surgery, 2015, http://www.datatrace.com/media/wysiwyg/pdf/Hip-TOC.pdf From the book: The Hip: Preservation, Replacement, and Revision by James Cashman, MD; Nitin Goyal, MD; Javad Parvizi, MD, FRCS (2015). 


\section{Appendix B - List of publications on smart carpet not already in the Thesis}

[1] F. Muheidat and H. W. Tyrer, "Can we make a carpet smart enough to detect falls?," 2016 38th Annual International Conference of the IEEE Engineering in Medicine and Biology Society (EMBC), Orlando, FL, USA, 2016, pp. 5356-5359. doi: 10.1109/EMBC.2016.7591937

[2] H. W. Tyrer, F. Muheidat. Estimating Gait Parameters Using a Floor-Based Array Personnel Detector, Alzheimer's Association International Conference (AAIC) 2017, London, UK (Accepted)

[3] F. Muheidat, H. W. Tyrer. Adding Intelligence to a Floor Based Array Personnel Detector, Poster, Alzheimer's Association International Conference (AAIC) 2016, Toronto, ON, Canada, July, 2016 


\section{VITA}

Fadi Muheidat, received Bachelor of Science degree from Jordan University of Science and Technology (JUST), Jordan in 2000, and the Masters of Science in Electrical and Computer Engineering the University of Missouri-Columbia (MU) in 2015, with a thesis titled "Adding Intelligence to a Floor based Array Personnel Detector". He worked for one year as a Software Developer and then worked for another year as a Network Engineer for an internet service provider. In 2002, He joined a group of System and Network engineers, and software developers to lead the Department of a fully integrated Information Technology System for a newly built hospital in Jordan. He was teaching assistant, teaching fellow and graduate instructor for variety of courses in Electrical and Computer Engineering Department at the University of Missouri. His research interests include eldercare technology, computational Intelligence, computer architecture, high performance computing, data analysis, and patient reported outcomes. 